\title{
Mixed Static and Dynamic Optimization of Four-Parameter Functionally Graded Completely Doubly Curved and Degenerate Shells and Panels Using GDQ Method
}

\author{
Francesco Tornabene $^{1}$ and Alessandro Ceruti $^{2}$ \\ ${ }^{1}$ DICAM-Department, School of Engineering, University of Bologna, viale del Risorgimento 2, 40136 Bologna, Italy \\ ${ }^{2}$ DIN-Department, School of Engineering, University of Bologna, viale del Risorgimento 2, 40136 Bologna, Italy
}

Correspondence should be addressed to Francesco Tornabene; francesco.tornabene@unibo.it

Received 14 February 2013; Accepted 2 April 2013

Academic Editor: Abdelouahed Tounsi

Copyright (C) 2013 F. Tornabene and A. Ceruti. This is an open access article distributed under the Creative Commons Attribution License, which permits unrestricted use, distribution, and reproduction in any medium, provided the original work is properly cited.

\begin{abstract}
This study deals with a mixed static and dynamic optimization of four-parameter functionally graded material (FGM) doubly curved shells and panels. The two constituent functionally graded shell consists of ceramic and metal, and the volume fraction profile of each lamina varies through the thickness of the shell according to a generalized power-law distribution. The Generalized Differential Quadrature (GDQ) method is applied to determine the static and dynamic responses for various FGM shell and panel structures. The mechanical model is based on the so-called First-order Shear Deformation Theory (FSDT). Three different optimization schemes and methodologies are implemented. The Particle Swarm Optimization, Monte Carlo and Genetic Algorithm approaches have been applied to define the optimum volume fraction profile for optimizing the first natural frequency and the maximum static deflection of the considered shell structure. The optimization aim is in fact to reach the frequency and the static deflection targets defined by the designer of the structure: the complete four-dimensional search space is considered for the optimization process. The optimized material profile obtained with the three methodologies is presented as a result of the optimization problem solved for each shell or panel structure.
\end{abstract}

\section{Introduction}

Shell structures are widely used in many fields of engineering thanks to their optimum dynamic behavior, strength, and stability guaranteed by the curvature effect. The dynamic and static deflection of these structures, caused by different external forces, can have serious consequences for their strength and safety, like resonance. Therefore, an accurate static deflection and frequency determination are of paramount importance for the technical design of these structural elements. One of the aims of this work is to study the static and dynamic behavior of completely doubly curved shell structures. During the last sixty years, twodimensional linear theories of thin shells and plates have been developed including important contributions [1-12]. The transverse shear deformation has been incorporated into shell theories by applying the theory of Reissner-Mindlin $[13,14]$, also named First-order Shear Deformation Theory (FSDT). Abandoning the assumption of the preservation of the normals to the shell middle surface after the deformation, a comprehensive analysis for elastic isotropic shells and plates was made by Kraus [7] and Gould $[15,16]$. Indeed, the present work is just based on the FSDT. In order to include the effect of the initial curvature in the evaluation of the stress resultants a generalization of the classical Reissner-Mindlin theory (CRMT) has been proposed in the literature by Kraus [7], by Leissa and Chang [17], by Qatu [18, 19] and by Toorani and Lakis [20]. As a consequence of these contributions 
the stress resultants directly depend on the geometry of the structure in terms of the curvature coefficients, and the hypothesis of the symmetry of the in-plane shearing force resultants and the torsional couples declines. A further improvement of the previous theories of shells has been proposed by Toorani and Lakis [21]. In the present work the kinematical model is generalized in order to include the effect of the curvatures from the beginning of the shell formulation. In this way, the strain relationships have been changed, and the equilibrium equations in terms of displacements have to be modified. The General First-order Shear Deformation Theory (GFSDT) is herein considered. It is worth noting that no results are available in the literature about this general theory for doubly curved shells. Thus, the motivation of the present work is based on the lack of results about completely doubly curved shells and panels.

Due to the significant developments that have taken place in composite materials [22], the laminated composite doubly curved shells and panels are considered in this work. As for the static and dynamic analysis of shells, several studies have been presented earlier [23]. Furthermore, some complicated effects in shell structures have been considered in the recent years $[24,25]$. Referring to the formulation of the static and dynamic equilibrium in terms of midsurface displacements and rotations, in this paper the system of second-order linear partial differential equations is solved. The static and dynamic solutions are obtained by using the numerical technique named Generalized Differential Quadrature (GDQ) method. The mathematical fundamentals and recent developments of the GDQ method as well as its major applications in engineering are discussed in detail in the book by Shu [26]. In the GDQ method the governing differential equations of equilibrium are directly transformed in one step to obtain the final algebraic form. The interest in researches dealing with this procedure is increasing due to its great simplicity and versatility. As shown in the literature [23, 27], GDQ technique is a global method, which can obtain very accurate numerical results by using a considerably small number of grid points. Therefore, this simple direct procedure has been applied in a large number of cases [28-77] to circumvent the difficulties of programming complex algorithms for the computer, as well as to avoid the excessive use of storage and computing time.

In the last decades, the increased use of functionally graded materials (FGMs) $[39,49-55,57-62,64,65,70,75,78$ 91] in engineering structures calls for improved analysis and tailored design tools. Thus, in the present paper, functionally graded shells are considered. Typically, FGMs consist of a mixture of ceramic and metal, or a combination of different materials. In this study, ceramic-metal graded shells and panels with two different volume fraction power-law variations of the constituents in the thickness direction are considered. Two different four-parameter power-law distributions, proposed by Tornabene [49], are used for the ceramic volume fraction. Various material profiles through the functionally graded lamina thickness are chosen by varying the four parameters of the power-law distributions.

The need for optimization lies in the mathematical formulation of FGMs, based on the four coefficients [49]: it is in fact quite difficult to handle these materials from the perspective of a designer. Small changes in parameters can lead in fact to strong changes in the distribution of base materials mixing in the thickness. Moreover, the domain of the four parameters is not continuous since some combinations of the parameters lead to distribution in which the mix of ceramics and metal resulting from the mathematical formula is negative or a complex number. From an operative perspective, a typical structural design scenario can include the need for minimizing the weight, the request of avoiding a resonance frequency, the control of the maximum displacement, or other constraints: in this case, there is no way to analytically relate the four parameter values to these important design requirements. As an answer, several authors proposed to apply optimization methods to achieve a feasible solution. For instance, Yas et al. [92] propose to minimize the weight of FGMs by using Imperialist Competitive Algorithm and Artificial Neural Networks. A very similar approach has been followed by Jam et al. [93] to optimize FGM conical shells. According to these examples, heuristic or semi heuristic methods are suitable in such a case since they consider the function to optimize as a "black box", in which only inputs and outputs are considered. A lot of heuristic (or semi heuristic) optimization techniques have been proposed in the literature. A comprehensive but not complete list includes Tabu Search [94], Simulated Annealing [95, 96], Ant Colonies Optimization [97, 98], Genetic Algorithms [99-109], Differential Evolution [110], Particle Swarm Algorithm [111-116], Immune Systems [117, 118], Gravity Optimization [119], Imperialist Competitive Algorithm [120], and Intelligent Water Drop [121, 122]. In this paper, Genetic Algorithm (GA) and Particle Swarm Optimization (PSO) will be used since they are tested, and widespread methods and other techniques could have been applied. Also an optimization with Monte Carlo (MC) technique $[115,123-127]$ will be presented to check the good performance of GA and PSO and to evaluate how much these methods are efficient and reliable with respect to a completely random approach. From the point of view of the software implementation, the PSO method is more simple than the GA, but it requires more attention to boundary conditions. MC is considered as a support to the GA and PSO techniques: in this paper, it is used to check the functionality of GA and PSO and to confirm that heuristic methods are to be preferred to random approaches. A well-designed algorithm implementing optimization techniques like GA or PSO can be useful to obtain a better solution than MC, requiring a shorter time to solve the optimization problem. MC is in fact a random technique, in which large number of tries is necessary to obtain a good result: moreover, the search is blind and without memory of the past, so that the previous tests of the optimization domain are not exploited by the following computations. The above considerations suggested the application of heuristic methods to the optimization of FGMs; a more detailed description of the implementation of these methods will be provided in the next sections of the present work.

The structure of this article is as follows: after this introduction, the second section describes the mathematical framework necessary to model and study FGM doubly 
curved shell structures; the third section shows the GDQ numerical implementation for the static and dynamic analyses; the fourth section describes the implementation of GA, PSO, and MC methods with respect to peculiarities of the case study, while the fifth one presents the results of the FGM optimization procedure applied to a set of six structures. A final section provides some conclusive comments regarding the problem description, the approach followed to solve it, and results obtained. The main novelty of the papers lies in the proposal of a design methodology which can be used by the designer involved in FGM applications to achieve a short time particular performances or characteristics for the FGM doubly curved shell structures.

\section{Geometry Description and Shell Fundamental Systems of Equations}

A 2D Equivalent Single Layer (ESL) model is proposed to study generic doubly curved shells and panels. The position of an arbitrary point within the shell medium is defined by coordinates $\alpha_{1}\left(\alpha_{1}^{0} \leq \alpha_{1} \leq \alpha_{1}^{1}\right), \alpha_{2}\left(\alpha_{2}^{0} \leq \alpha_{2} \leq \alpha_{2}^{1}\right)$ upon the middle surface or reference surface $\mathbf{r}\left(\alpha_{1}, \alpha_{2}\right)$, and $\zeta$ directed along the outward normal $\mathbf{n}\left(\alpha_{1}, \alpha_{2}\right)$ and measured from the reference surface $(-h / 2 \leq \zeta \leq h / 2)$, where $h\left(\alpha_{1}, \alpha_{2}\right)$ is the total thickness of the shell (Figure 1). The differential geometry $[7,11,12,23,74]$ is used to describe the shell structure, starting with the position vector written in the global reference system:

$$
\mathbf{R}\left(\alpha_{1}, \alpha_{2}, \zeta\right)=\mathbf{r}\left(\alpha_{1}, \alpha_{2}\right)+\frac{h\left(\alpha_{1}, \alpha_{2}\right)}{2} z \mathbf{n}\left(\alpha_{1}, \alpha_{2}\right),
$$

where $z=2 \zeta / h\left(\alpha_{1}, \alpha_{2}\right)$ and $z \in[-1,1]$. The basic configuration of the problem considered is a laminated composite doubly curved shell, as shown in Figure 1. For a laminated composite shell made of $l$ laminae or plies, the total thickness $h$ is defined as

$$
h=\sum_{k=1}^{l} h_{k}
$$

in which $h_{k}=\zeta_{k+1}-\zeta_{k}$ is the thickness of the $k$ th lamina or ply. In this study, doubly curved shells and degenerate shells such as plates are considered. It should be noted from (1) that the location of each point of the 3D shell is a function of the location of the point on the reference surface $\mathbf{r}\left(\alpha_{1}, \alpha_{2}\right)$ and the normal vector $\mathbf{n}\left(\alpha_{1}, \alpha_{2}\right)$ to the reference surface at the given point (Figure 1). Moreover, the position of the generic point of the shell volume is also a function of the shell thickness $h\left(\alpha_{1}, \alpha_{2}\right)$. Hence, writing the position vector of the reference surface, it is possible to define the three components along the three global axes $O x_{1} x_{2} x_{3}$ as

$$
\mathbf{r}\left(\alpha_{1}, \alpha_{2}\right)=r_{1}\left(\alpha_{1}, \alpha_{2}\right) \mathbf{e}_{1}+r_{2}\left(\alpha_{1}, \alpha_{2}\right) \mathbf{e}_{2}+r_{3}\left(\alpha_{1}, \alpha_{2}\right) \mathbf{e}_{3},
$$

where $\mathbf{e}_{1}, \mathbf{e}_{2}$, and $\mathbf{e}_{3}$ are the unit vector of the global reference system $O x_{1} x_{2} x_{3}$. From the definition of the first fundamental form $[7,11,12,23]$ of the reference surface $\mathbf{r}\left(\alpha_{1}, \alpha_{2}\right)$, the Lamé parameters can be expressed as

$$
\begin{aligned}
& A_{1}\left(\alpha_{1}, \alpha_{2}\right)=\sqrt{\mathbf{r}_{, 1} \cdot \mathbf{r}_{, 1}}, \\
& A_{2}\left(\alpha_{1}, \alpha_{2}\right)=\sqrt{\mathbf{r}_{, 2} \cdot \mathbf{r}_{, 2}} .
\end{aligned}
$$

The comma in expressions (4) defines the partial derivative with respect to $\alpha_{1}$ or $\alpha_{2}$, respectively. Moreover, by considering an orthogonal curvilinear coordinate system $O^{\prime} \alpha_{1} \alpha_{2} \zeta$ $[7,11,12,23,74]$ from the position vector (3) the normal vector $\mathbf{n}\left(\alpha_{1}, \alpha_{2}\right)$ can be written as

$$
\mathbf{n}\left(\alpha_{1}, \alpha_{2}\right)=\frac{\mathbf{r}_{, 1} \times \mathbf{r}_{, 2}}{A_{1} A_{2}} .
$$

Finally, due to the fact that an orthogonal curvilinear coordinate system $O^{\prime} \alpha_{1} \alpha_{2} \zeta$ is considered and following the definition of the second fundamental form $[7,11,23,74]$ of the reference surface $\mathbf{r}\left(\alpha_{1}, \alpha_{2}\right)$, the principal radii of curvature can be evaluated as

$$
\begin{aligned}
& R_{1}\left(\alpha_{1}, \alpha_{2}\right)=-\frac{\mathbf{r}_{, 1} \cdot \mathbf{r}_{, 1}}{\mathbf{r}_{, 11} \cdot \mathbf{n}}, \\
& R_{2}\left(\alpha_{1}, \alpha_{2}\right)=-\frac{\mathbf{r}_{, 2} \cdot \mathbf{r}_{2,}}{\mathbf{r}_{, 22} \cdot \mathbf{n}} .
\end{aligned}
$$

As concerns the shell theory, the present study is based on the following assumptions: (1) the transverse normal is inextensible so that the normal strain is equal to zero: $\varepsilon_{n}=$ $\varepsilon_{n}\left(\alpha_{1}, \alpha_{2}, \zeta, t\right)=0$; (2) the transverse shear deformation is considered to influence the governing equations so that normal lines to the reference surface of the shell before deformation remain straight but not necessarily normal after deformation (a relaxed Kirchhoff-Love hypothesis is considered); (3) the shell deflections are small and the strains are infinitesimal; (4) the shell is moderately thick, therefore it is possible to assume that the thickness-direction normal stress is negligible so that the plane assumption can be invoked: $\sigma_{n}=\sigma_{n}\left(\alpha_{1}, \alpha_{2}, \zeta, t\right)=0$; (5) the linear elastic behavior of anisotropic materials is assumed; and (6) the rotary inertias and the initial curvatures are also taken into account. The theory under development is meant to be in the group of moderately thick shells, in which the following ratios of thickness over curvature and curve length are valid:

$$
\frac{1}{100} \leq \max \left(\frac{h}{R_{\min }}, \frac{h}{L_{\min }}\right) \leq \frac{1}{10} .
$$

Consistent with the assumptions of a moderately thick shell theory reported above, the displacement field considered in the present study is that of the First-order Shear Deformation Theory (FSDT) and can be put in the following form:

$$
\begin{gathered}
U_{1}\left(\alpha_{1}, \alpha_{2}, \zeta, t\right)=H_{1} u_{1}\left(\alpha_{1}, \alpha_{2}, t\right)+\zeta \beta_{1}\left(\alpha_{1}, \alpha_{2}, t\right), \\
U_{2}\left(\alpha_{1}, \alpha_{2}, \zeta, t\right)=H_{2} u_{2}\left(\alpha_{1}, \alpha_{2}, t\right)+\zeta \beta_{2}\left(\alpha_{1}, \alpha_{2}, t\right), \\
U_{3}\left(\alpha_{1}, \alpha_{2}, \zeta, t\right)=u_{3}\left(\alpha_{1}, \alpha_{2}, t\right),
\end{gathered}
$$


where

$$
\begin{aligned}
& H_{1}=1+\frac{\zeta}{R_{1}}, \\
& H_{2}=1+\frac{\zeta}{R_{2}},
\end{aligned}
$$

and $u_{1}, u_{2}$, and $u_{3}$ are the displacement components of points lying on the middle surface $(\zeta=0)$ of the shell, while $t$ is the time variable. $\beta_{1}$ and $\beta_{2}$ are normal-to-mid-surface rotations, respectively. The kinematic hypothesis expressed by relations ( 8 ) should be supplemented by the statement that the shell deflections are small and strains are infinitesimal, that is $u_{3} \ll h$. In-plane displacements $U_{1}$ and $U_{2}$ vary linearly through the thickness, while $U_{3}$ remains independent of $\zeta$. Differently from the previous works $[43,44,47,49-$ $53,67,68,70]$, the displacement field has been improved taking into account the effective geometry of the shell and in particular the curvature effect has been directly introduced into the kinematical model, as proposed by Toorani and Lakis [21].

Due to the change of the kinematical model, the relationships between strains and generalized displacements along the shell reference surface $(\zeta=0)$ become the following:

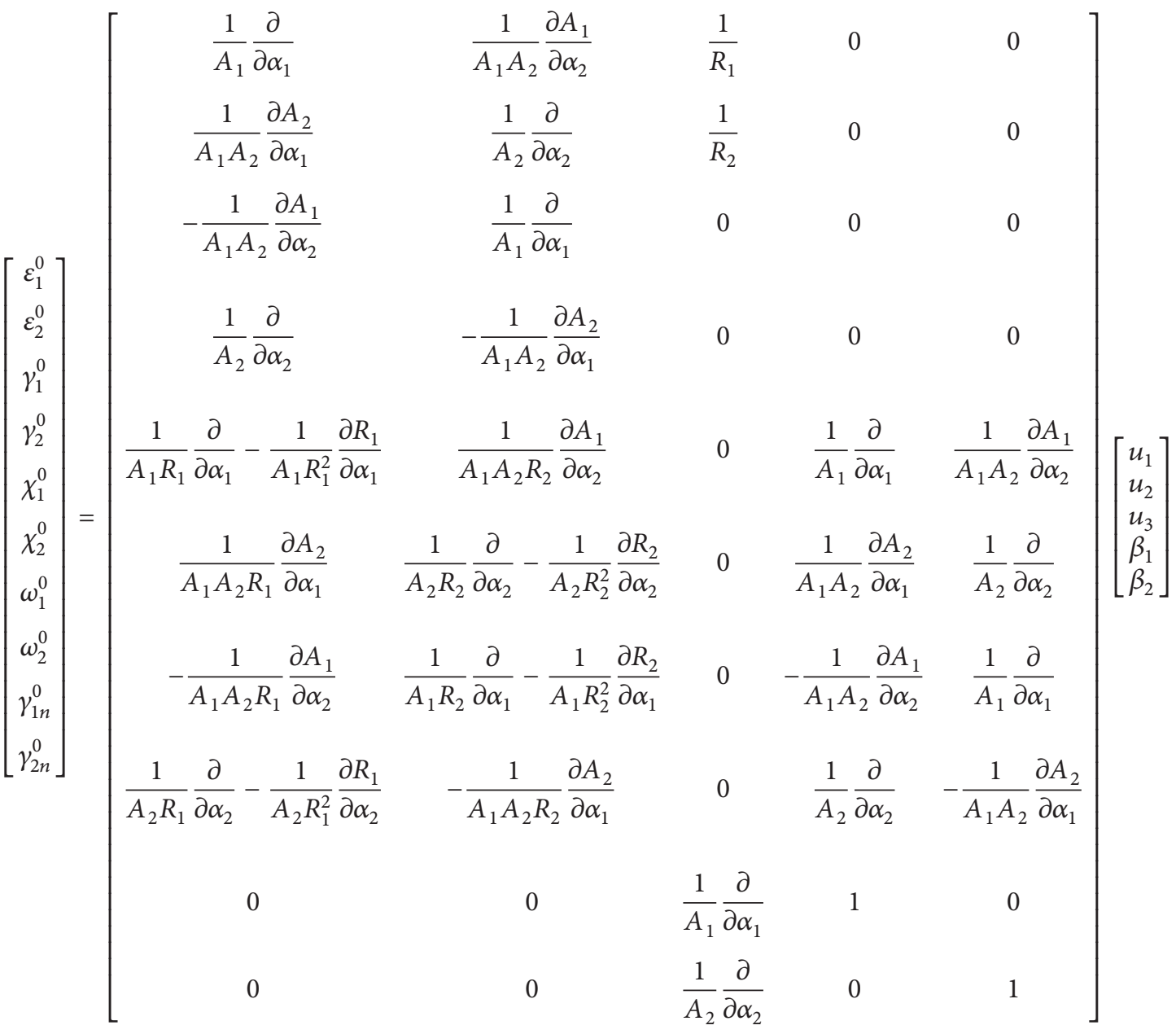

that are different from those presented in previous papers $[43,44,47,49-53,67,68,70]$. In the above (10), the first four strains $\varepsilon_{1}^{0}, \varepsilon_{2}^{0}, \gamma_{1}^{0}$, and $\gamma_{2}^{0}$ are the in-plane components, and $\chi_{1}^{0}, \chi_{2}^{0}, \omega_{1}^{0}$, and $\omega_{2}^{0}$ are the analogous curvature changes. The last two components $\gamma_{1 n}^{0}, \gamma_{2 n}^{0}$ are the transverse shearing strains. The shell is assumed to be made of a linear elastic composite material. Accordingly, the following constitutive equations relate internal stress resultants and internal couples with generalized strain components (10) on the middle surface: 


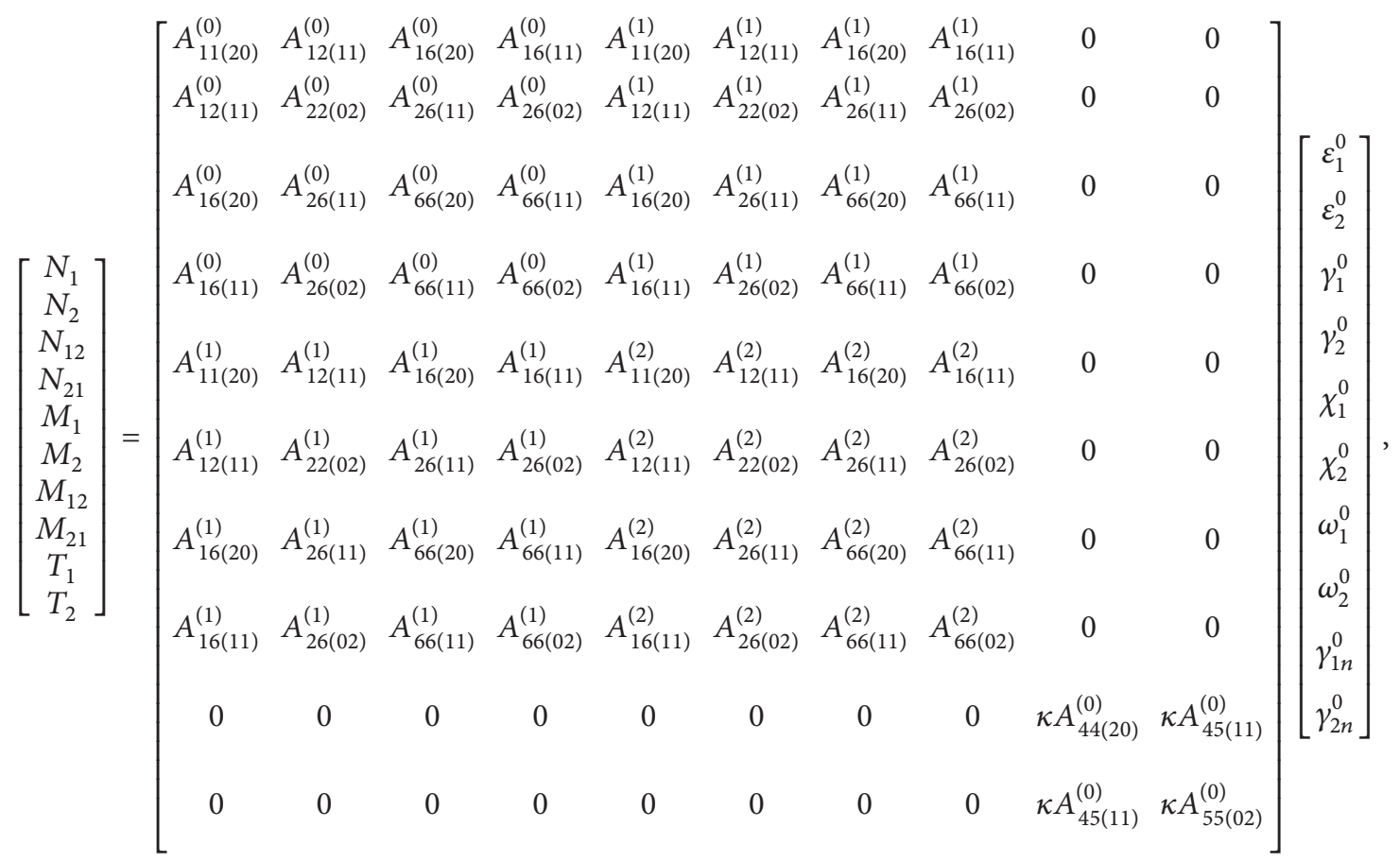

where the elastic engineering stiffnesses $A_{i j(p q)}^{(\tau)}$ are defined as follows:

$$
A_{i j(p q)}^{(\tau)}=\sum_{k=1}^{l} \int_{\zeta_{k}}^{\zeta_{k+1}} \bar{Q}_{i j}^{(k)} \zeta^{\tau} \frac{H_{1} H_{2}}{H_{1}^{p} H_{2}^{q}} d \zeta, \quad \tau, p, q=0,1,2 .
$$

Different approaches can be found in the literature to evaluate the engineering elastic constants $A_{i j(p q)}^{(\tau)}[7,17-20,73,76]$. In the present paper, the relations of the elastic engineering stiffnesses $A_{i j(p q)}^{(\tau)}$ are numerically evaluated using an integral function in order to avoid numerical instabilities. It is worth noting that due to the fact that the elastic engineering stiffnesses $A_{i j(p q)}^{(\tau)}$ depend on curvatures, the corresponding derivatives, with respect to the coordinates along $\alpha_{1}$ and $\alpha_{2}$ directions of the reference surface, have to be evaluated. In order to perform this operation the Differential Quadrature rule [26] is used. Thus, the derivatives of the elastic engineering stiffnesses $A_{i j(p q)}^{(\tau)}$ are numerically evaluated. The corresponding elastic constants $\bar{Q}_{i j}^{(k)}$ can be found in the article by Tornabene et al. $[70,73]$, in which all the constants above introduced are explicitly defined for laminated composite and functionally graded shells and panels. $\kappa$ is the shear correction factor, which is usually taken as $\kappa=5 / 6$, such as in the present work. In particular, the determination of shear correction factors for composite laminated structures is still an unresolved issue, since these factors depend on various parameters [20]. In (11), the four components $N_{1}, N_{2}, N_{12}$, and $N_{21}$ are the in-plane force resultants, and $M_{1}, M_{2}, M_{12}$, and $M_{21}$ are the analogous couples, while $T_{1}, T_{2}$ are the transverse shear force resultants. In the above definitions
(11) the symmetry of shearing force resultants $N_{12}, N_{21}$ and torsional couples $M_{12}, M_{21}$ is not assumed as a further hypothesis, as done in Reissner-Mindlin theory [7, 12, 22]. This hypothesis is satisfied only in the case of spherical shells and flat plates [7]. The assumption under discussion is derived from the consideration that ratios $\zeta / R_{1}, \zeta / R_{2}$ cannot be neglected with respect to unity.

Typically, the functionally graded materials are made of a mixture of two constituents. In the present work, it is assumed that the functionally graded material lamina is made of a mixture of ceramic and metal constituents: Silicon Nitride and Stainless Steel. The material properties of the functionally graded shell vary continuously and smoothly in the thickness direction $\zeta$ of each lamina and are functions of volume fractions of two constituent materials. The Young's modulus $E^{(k)}(\zeta)$, shear modulus $G^{(k)}(\zeta)$, Poisson's ratio $v^{(k)}(\zeta)$, and mass density $\rho^{(k)}(\zeta)$ of the functionally graded shell $k$ th lamina can be expressed as

$$
\begin{aligned}
\rho^{(k)}(\zeta) & =\left(\rho_{C}^{(k)}-\rho_{M}^{(k)}\right) V_{C}^{(k)}(\zeta)+\rho_{M}^{(k)}, E^{(k)}(\zeta) \\
& =\left(E_{C}^{(k)}-E_{M}^{(k)}\right) V_{C}^{(k)}(\zeta)+E_{M}^{(k)}, v^{(k)}(\zeta) \\
& =\left(\nu_{C}^{(k)}-v_{M}^{(k)}\right) V_{C}^{(k)}(\zeta)+v_{M}^{(k)}, G^{(k)}(\zeta) \\
& =\frac{E^{(k)}(\zeta)}{2\left(1+v^{(k)}(\zeta)\right)}, \quad \text { for } \zeta_{k} \leq \zeta \leq \zeta_{k+1},
\end{aligned}
$$

where $\rho_{C}^{(k)}, E_{C}^{(k)}, v_{C}^{(k)}, V_{C}^{(k)}$ and $\rho_{M}^{(k)}, E_{M}^{(k)}, v_{M}^{(k)}, V_{M}^{(k)}$ represent mass density, Young's modulus, Poisson's ratio, and volume fraction of the ceramic and metal constituent materials, respectively. In this work, the ceramic volume fraction $V_{C}^{(k)}(\zeta)$ 
follows two simple four-parameter power-law distributions $[49,51,53,70,75]$ :

$$
\begin{aligned}
& \mathrm{FGM}_{1\left(a^{(k)} / b^{(k)} / c^{(k)} / p^{(k)}\right)}: V_{C}^{(k)}(\zeta) \\
& =\left(1-a^{(k)}\left(\frac{\zeta}{h_{k}}-\frac{\zeta_{k}}{h_{k}}\right)+b^{(k)}\left(\frac{\zeta}{h_{k}}-\frac{\zeta_{k}}{h_{k}}\right)^{c^{(k)}}\right)^{p^{(k)}}, \\
& \mathrm{FGM}_{2\left(a^{(k)} / b^{(k)} / c^{(k)} / p^{(k)}\right)}: V_{C}^{(k)}(\zeta) \\
& =\left(1-a^{(k)}\left(\frac{\zeta_{k+1}}{h_{k}}-\frac{\zeta}{h_{k}}\right)+b^{(k)}\left(\frac{\zeta_{k+1}}{h_{k}}-\frac{\zeta}{h_{k}}\right)^{c^{(k)}}\right)^{p^{(k)}},
\end{aligned}
$$

where the volume fraction index $p^{(k)}\left(0 \leq p^{(k)} \leq \infty\right)$ and the parameters $a^{(k)}, b^{(k)}$, and $c^{(k)}$ dictate the material variation profile through the functionally graded shell lamina thickness. It is important to remark that the volume fractions of all the constituent materials should add up to unity:

$$
V_{C}^{(k)}+V_{M}^{(k)}=1
$$

In order to choose the three parameters $a^{(k)}, b^{(k)}$, and $c^{(k)}$ suitably, the relation (15) must be always satisfied for every volume fraction index $p^{(k)}$ in each lamina. By considering the relations (14), when the power-law exponent is set equal to zero $\left(p^{(k)}=0\right)$ or equal to infinity $\left(p^{(k)}=\infty\right)$, the homogeneous isotropic material is obtained as a special case of functionally graded material. In fact, from (15), (14), and (13) it is possible to obtain

$$
\begin{aligned}
p^{(k)}=0 \longrightarrow V_{C}^{(k)}=1, & V_{M}^{(k)}=0 \longrightarrow \rho^{(k)}(\zeta)=\rho_{C}, \\
E^{(k)}(\zeta)=E_{C}, & v^{(k)}(\zeta)=\nu_{C}, \\
p^{(k)}=\infty \longrightarrow V_{C}^{(k)}=0, & V_{M}^{(k)}=1 \longrightarrow \rho^{(k)}(\zeta)=\rho_{M}, \\
E^{(k)}(\zeta)=E_{M}, \quad v^{(k)}(\zeta) & =v_{M} .
\end{aligned}
$$

Some material profiles through the functionally graded shell thickness are illustrated in Figures 2 and 3.
Following the Hamilton's principle [7, 12, 19, 22, 23], the five governing equations in terms of internal actions can be written for the shell element:

$$
\begin{gathered}
\frac{1}{A_{1} A_{2}} \frac{\partial\left(N_{1} A_{2}\right)}{\partial \alpha_{1}}+\frac{1}{A_{1} A_{2}} \frac{\partial\left(N_{21} A_{1}\right)}{\partial \alpha_{2}} \\
+\frac{N_{12}}{A_{1} A_{2}} \frac{\partial A_{1}}{\partial \alpha_{2}}-\frac{N_{2}}{A_{1} A_{2}} \frac{\partial A_{2}}{\partial \alpha_{1}} \\
+\frac{1}{R_{1}}\left(\frac{1}{A_{1} A_{2}} \frac{\partial\left(M_{1} A_{2}\right)}{\partial \alpha_{1}}\right. \\
+\frac{1}{A_{1} A_{2}} \frac{\partial\left(M_{21} A_{1}\right)}{\partial \alpha_{2}} \\
\left.+\frac{M_{12}}{A_{1} A_{2}} \frac{\partial A_{1}}{\partial \alpha_{2}}-\frac{M_{2}}{A_{1} A_{2}} \frac{\partial A_{2}}{\partial \alpha_{1}}\right)
\end{gathered}
$$$$
+q_{1}=\left(I_{0}+\frac{2 I_{1}}{R_{1}}+\frac{I_{2}}{R_{1}^{2}}\right) \ddot{u}_{1}+\left(I_{1}+\frac{I_{2}}{R_{1}}\right) \ddot{\beta}_{1} \text {, }
$$$$
\frac{1}{A_{1} A_{2}} \frac{\partial\left(N_{12} A_{2}\right)}{\partial \alpha_{1}}+\frac{1}{A_{1} A_{2}} \frac{\partial\left(N_{2} A_{1}\right)}{\partial \alpha_{2}}
$$$$
+\frac{N_{21}}{A_{1} A_{2}} \frac{\partial A_{2}}{\partial \alpha_{1}}-\frac{N_{1}}{A_{1} A_{2}} \frac{\partial A_{1}}{\partial \alpha_{2}}
$$$$
+\frac{1}{R_{2}}\left(\frac{1}{A_{1} A_{2}} \frac{\partial\left(M_{12} A_{2}\right)}{\partial \alpha_{1}}\right.
$$$$
+\frac{1}{A_{1} A_{2}} \frac{\partial\left(M_{2} A_{1}\right)}{\partial \alpha_{2}}
$$$$
\left.+\frac{M_{21}}{A_{1} A_{2}} \frac{\partial A_{2}}{\partial \alpha_{1}}-\frac{M_{1}}{A_{1} A_{2}} \frac{\partial A_{1}}{\partial \alpha_{2}}\right)
$$$$
+q_{2}=\left(I_{0}+\frac{2 I_{1}}{R_{2}}+\frac{I_{2}}{R_{2}^{2}}\right) \ddot{u}_{2}+\left(I_{1}+\frac{I_{2}}{R_{2}}\right) \ddot{\beta}_{2} \text {, }
$$$$
\frac{1}{A_{1} A_{2}} \frac{\partial\left(T_{1} A_{2}\right)}{\partial \alpha_{1}}+\frac{1}{A_{1} A_{2}} \frac{\partial\left(T_{2} A_{1}\right)}{\partial \alpha_{2}}
$$$$
-\left(\frac{N_{1}}{R_{1}}+\frac{N_{2}}{R_{2}}\right)+q_{n}=I_{0} \ddot{u}_{3},
$$

$$
\begin{gathered}
\frac{1}{A_{1} A_{2}} \frac{\partial\left(M_{1} A_{2}\right)}{\partial \alpha_{1}}+\frac{1}{A_{1} A_{2}} \frac{\partial\left(M_{21} A_{1}\right)}{\partial \alpha_{2}}+\frac{M_{12}}{A_{1} A_{2}} \frac{\partial A_{1}}{\partial \alpha_{2}} \\
-\frac{M_{2}}{A_{1} A_{2}} \frac{\partial A_{2}}{\partial \alpha_{1}}-T_{1}+m_{1}=\left(I_{1}+\frac{I_{2}}{R_{1}}\right) \ddot{u}_{1}+I_{2} \ddot{\beta}_{1},
\end{gathered}
$$

$$
\begin{gathered}
\frac{1}{A_{1} A_{2}} \frac{\partial\left(M_{12} A_{2}\right)}{\partial \alpha_{1}}+\frac{1}{A_{1} A_{2}} \frac{\partial\left(M_{2} A_{1}\right)}{\partial \alpha_{2}}+\frac{M_{21}}{A_{1} A_{2}} \frac{\partial A_{2}}{\partial \alpha_{1}} \\
-\frac{M_{1}}{A_{1} A_{2}} \frac{\partial A_{1}}{\partial \alpha_{2}}-T_{2}+m_{2}=\left(I_{1}+\frac{I_{2}}{R_{2}}\right) \ddot{u}_{2}+I_{2} \ddot{\beta}_{2}
\end{gathered}
$$




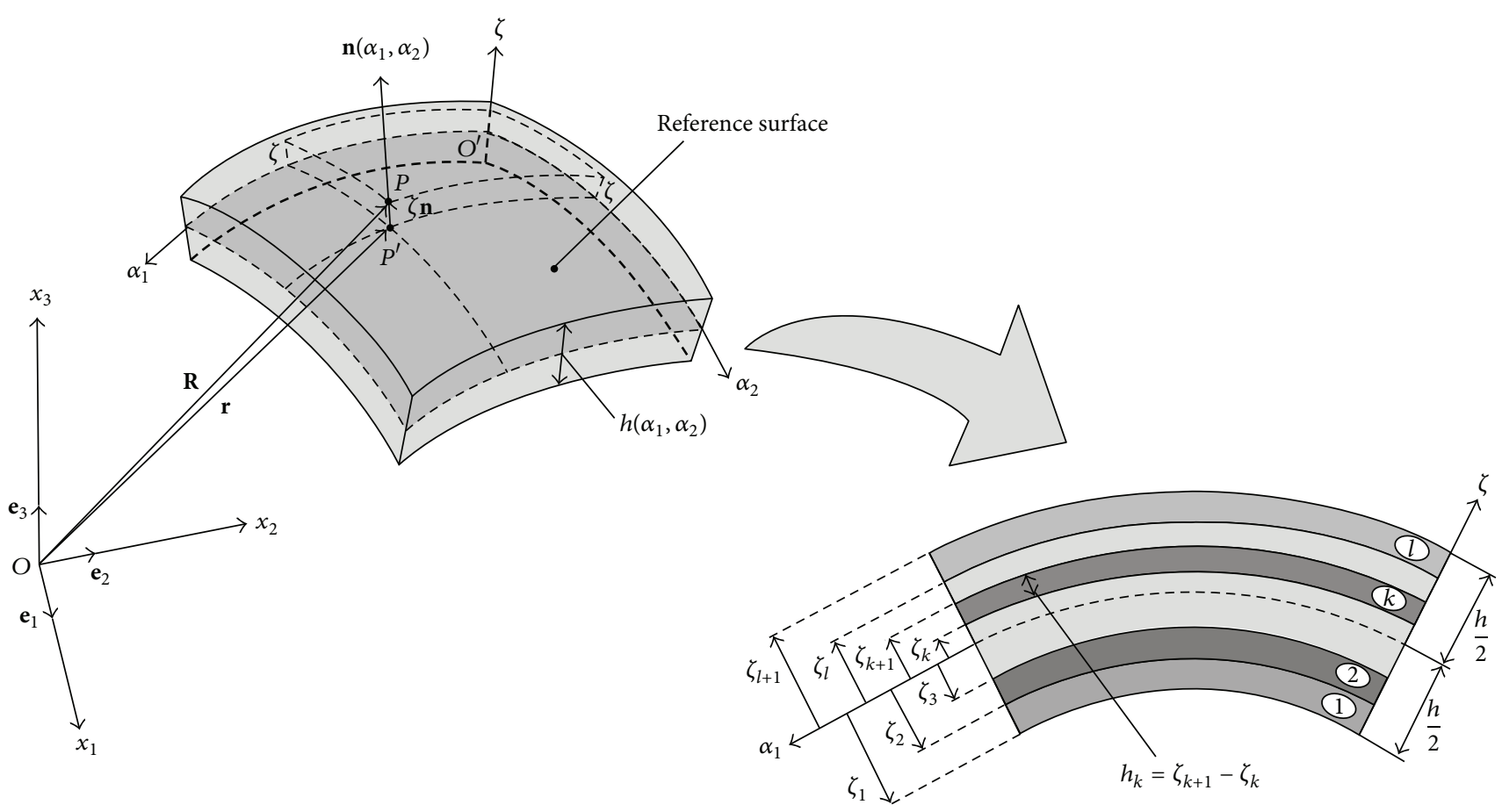

FIGURE 1: Geometry description and coordinate system of a doubly curved shell.

where

$$
I_{\tau}=\sum_{k=1}^{l} \int_{\zeta_{k}}^{\zeta_{k+1}} \rho^{(k)} \zeta^{\tau} H_{1} H_{2} d \zeta, \quad \tau=0,1,2
$$

Furthermore, the generalized external actions $q_{1}, q_{2}, q_{n}, m_{1}$, and $m_{2}$ due to the external forces, acting on the top and bottom surfaces of the shell, can be evaluated using the static equivalence principle $[7,23]$ and can be written on the reference surface of the doubly curved shell as follows:

$$
\begin{aligned}
q_{1}= & q_{1}^{+}\left(1+\frac{h}{2 R_{1}}\right)^{2}\left(1+\frac{h}{2 R_{2}}\right) \\
& +q_{1}^{-}\left(1-\frac{h}{2 R_{1}}\right)^{2}\left(1-\frac{h}{2 R_{2}}\right), \\
q_{2}= & q_{2}^{+}\left(1+\frac{h}{2 R_{1}}\right)\left(1+\frac{h}{2 R_{2}}\right)^{2} \\
& +q_{2}^{-}\left(1-\frac{h}{2 R_{1}}\right)\left(1-\frac{h}{2 R_{2}}\right)^{2},
\end{aligned}
$$

$$
\begin{aligned}
q_{n}= & q_{n}^{+}\left(1+\frac{h}{2 R_{1}}\right)\left(1+\frac{h}{2 R_{2}}\right) \\
& +q_{n}^{-}\left(1-\frac{h}{2 R_{1}}\right)\left(1-\frac{h}{2 R_{2}}\right), \\
m_{1}= & q_{1}^{+} \frac{h}{2}\left(1+\frac{h}{2 R_{1}}\right)\left(1+\frac{h}{2 R_{2}}\right) \\
& -q_{1}^{-} \frac{h}{2}\left(1-\frac{h}{2 R_{1}}\right)\left(1-\frac{h}{2 R_{2}}\right), \\
m_{2}= & q_{2}^{+} \frac{h}{2}\left(1+\frac{h}{2 R_{1}}\right)\left(1+\frac{h}{2 R_{2}}\right) \\
& -q_{2}^{-} \frac{h}{2}\left(1-\frac{h}{2 R_{1}}\right)\left(1-\frac{h}{2 R_{2}}\right),
\end{aligned}
$$

where $q_{1}^{+}, q_{1}^{-}, q_{2}^{+}, q_{2}^{-}, q_{n}^{+}$, and $q_{n}^{-}$are the external forces in the three principal directions $\alpha_{1}, \alpha_{2}$, and $\zeta$ at the top and the bottom surface of the shell, respectively. The three basic sets of equations, namely, the kinematic (10), constitutive (11), and motion (17) equations, may be combined to give the fundamental system of equations, also known as the governing system of equations. By replacing the kinematic equations (10) into the constitutive equations (11) and the result of this substitution into the motion equations (17), the complete equations of motion in terms of displacement and rotational components can be written as 


$$
\left[\begin{array}{lllll}
L_{11} & L_{12} & L_{13} & L_{14} & L_{15} \\
L_{21} & L_{22} & L_{23} & L_{24} & L_{25} \\
L_{31} & L_{32} & L_{33} & L_{34} & L_{35} \\
L_{41} & L_{42} & L_{43} & L_{44} & L_{45} \\
L_{51} & L_{52} & L_{53} & L_{54} & L_{55}
\end{array}\right]\left[\begin{array}{l}
u_{1} \\
u_{2} \\
u_{3} \\
\beta_{1} \\
\beta_{2}
\end{array}\right]+\left[\begin{array}{l}
q_{1} \\
q_{2} \\
q_{n} \\
m_{1} \\
m_{2}
\end{array}\right]=\left[\begin{array}{ccccc}
I_{01} & 0 & 0 & I_{11} & 0 \\
0 & I_{02} & 0 & 0 & I_{12} \\
0 & 0 & I_{0} & 0 & 0 \\
I_{11} & 0 & 0 & I_{2} & 0 \\
0 & I_{12} & 0 & 0 & I_{2}
\end{array}\right]\left[\begin{array}{l}
\ddot{u}_{1} \\
\ddot{u}_{2} \\
\ddot{u}_{3} \\
\ddot{\beta}_{1} \\
\ddot{\beta}_{2}
\end{array}\right]
$$

where $L_{i j}, i, j=1, \ldots, 5$ are the equilibrium operators and the new mass inertias are defined as follows:

$$
\begin{gathered}
I_{01}=I_{0}+\frac{2 I_{1}}{R_{1}}+\frac{I_{2}}{R_{1}^{2}}, \\
I_{11}=I_{1}+\frac{I_{2}}{R_{1}}, \\
I_{02}=I_{0}+\frac{2 I_{1}}{R_{2}}+\frac{I_{2}}{R_{2}^{2}}, \\
I_{12}=I_{1}+\frac{I_{2}}{R_{2}} .
\end{gathered}
$$

Differently from previous works $[43,44,47,49-53,67,68$, 70], the equilibrium operators $L_{i j}$, introduced in (20), have been changed due to the choice of using the kinematical model (8).

Three kinds of boundary conditions are considered, namely, the fully clamped edge boundary condition $(C)$, the simply supported edge boundary condition $(S)$, and the free edge boundary condition $(F)$. The equations describing the boundary conditions can be written as follows:

\section{Clamped edge boundary conditions $(C)$ :}

$$
\begin{gathered}
u_{1}=u_{2}=u_{3}=\beta_{1}=\beta_{2}=0 \text { at } \alpha_{1}=\alpha_{1}^{0} \\
\text { or } \alpha_{1}=\alpha_{1}^{1}, \alpha_{2}^{0} \leq \alpha_{2} \leq \alpha_{2}^{1}, \\
u_{1}=u_{2}=u_{3}=\beta_{1}=\beta_{2}=0 \text { at } \alpha_{2}=\alpha_{2}^{0} \\
\text { or } \alpha_{2}=\alpha_{2}^{1}, \alpha_{1}^{0} \leq \alpha_{1} \leq \alpha_{1}^{1},
\end{gathered}
$$

Simply supported edge boundary conditions (S):

$$
\begin{gathered}
u_{1}=u_{2}=u_{3}=\beta_{2}=0, \quad M_{1}=0 \quad \text { at } \alpha_{1}=\alpha_{1}^{0} \\
\text { or } \alpha_{1}=\alpha_{1}^{1}, \alpha_{2}^{0} \leq \alpha_{2} \leq \alpha_{2}^{1}, \\
u_{1}=u_{2}=u_{3}=\beta_{2}=0, \quad M_{2}=0 \quad \text { at } \alpha_{2}=\alpha_{2}^{0} \\
\text { or } \alpha_{2}=\alpha_{2}^{1}, \alpha_{1}^{0} \leq \alpha_{1} \leq \alpha_{1}^{1},
\end{gathered}
$$

Free edge boundary conditions $(F)$ :

$$
\begin{aligned}
& N_{1}+\frac{M_{1}}{R_{1}}=0, \quad N_{12}+\frac{M_{12}}{R_{2}}=0, \\
& T_{1}=0, \quad M_{1}=M_{12}=0 \quad \text { at } \alpha_{1}=\alpha_{1}^{0} \\
& \quad \text { or } \alpha_{1}=\alpha_{1}^{1}, \alpha_{2}^{0} \leq \alpha_{2} \leq \alpha_{2}^{1}, \\
& N_{2}+\frac{M_{2}}{R_{2}}=0, \quad N_{21}+\frac{M_{21}}{R_{1}}=0, \\
& T_{2}=0, \quad M_{2}=M_{21}=0 \quad \text { at } \alpha_{2}=\alpha_{2}^{0} \\
& \text { or } \alpha_{2}=\alpha_{2}^{1}, \alpha_{1}^{0} \leq \alpha_{1} \leq \alpha_{1}^{1} .
\end{aligned}
$$

In addition to the external boundary conditions (22)-(25), the kinematic and physical compatibility conditions should be satisfied at the common closing meridians with $\alpha_{2}=0,2 \pi$, if a complete shell of revolution has to be considered. The kinematic compatibility conditions include the continuity of displacements. The physical compatibility conditions can only be represented by the five continuous conditions for the generalized stress resultants. To consider complete revolute shells characterized by $\alpha_{2}^{1}=2 \pi$, it is necessary to implement the kinematic and physical compatibility conditions between the two computational meridians with $\alpha_{2}^{0}=0$ and with $\alpha_{2}^{1}=$ $2 \pi$ :

Kinematic compatibility conditions along the closing meridian $\left(\alpha_{2}=0,2 \pi\right)$

$$
\begin{array}{lr}
u_{1}\left(\alpha_{1}, 0, t\right)=u_{1}\left(\alpha_{1}, 2 \pi, t\right), & u_{2}\left(\alpha_{1}, 0, t\right)=u_{2}\left(\alpha_{1}, 2 \pi, t\right), \\
u_{3}\left(\alpha_{1}, 0, t\right)=u_{3}\left(\alpha_{1}, 2 \pi, t\right), & \\
\beta_{1}\left(\alpha_{1}, 0, t\right)=\beta_{1}\left(\alpha_{1}, 2 \pi, t\right), & \beta_{2}\left(\alpha_{1}, 0, t\right)=\beta_{2}\left(\alpha_{1}, 2 \pi, t\right) \\
& \alpha_{1}^{0} \leq \alpha_{1} \leq \alpha_{1}^{1},
\end{array}
$$

Physical compatibility conditions along the closing meridian $\left(\alpha_{2}=0,2 \pi\right)$

$$
\begin{aligned}
& N_{2}\left(\alpha_{1}, 0, t\right)+\frac{M_{2}\left(\alpha_{1}, 0, t\right)}{R_{2}} \\
& \quad=N_{2}\left(\alpha_{1}, 2 \pi, t\right)+\frac{M_{2}\left(\alpha_{1}, 2 \pi, t\right)}{R_{2}},
\end{aligned}
$$




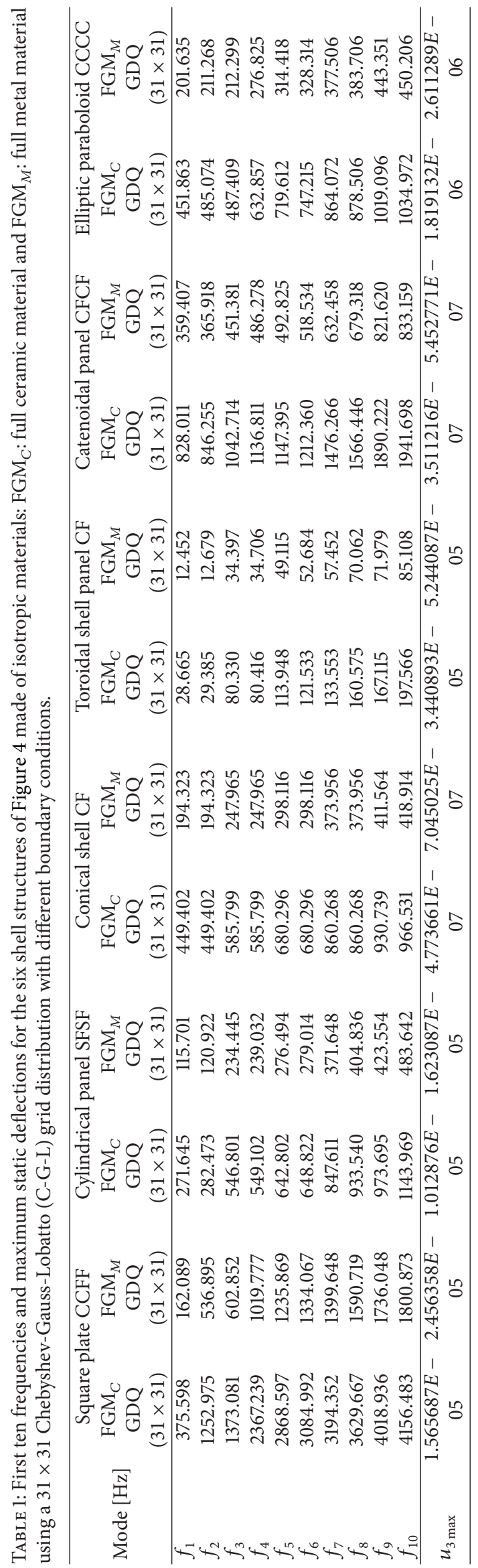




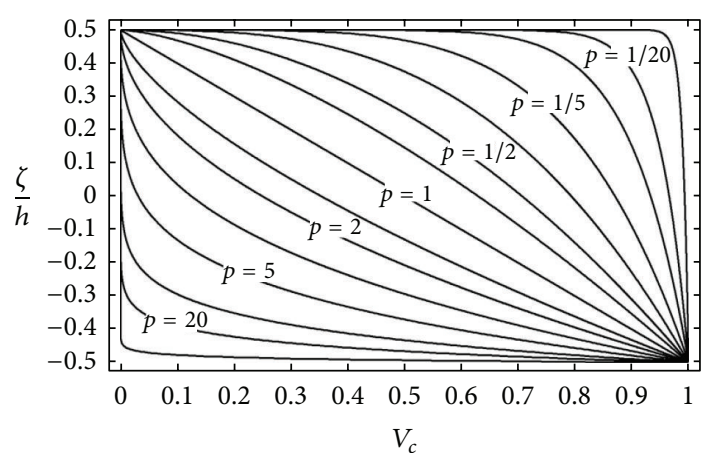

(a)

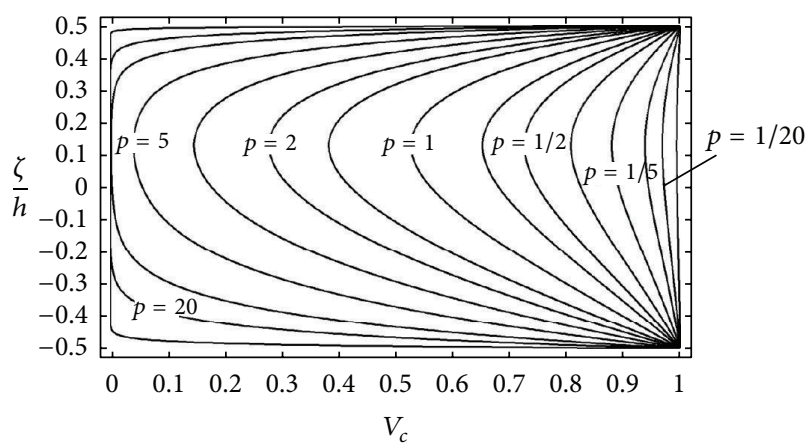

(c)

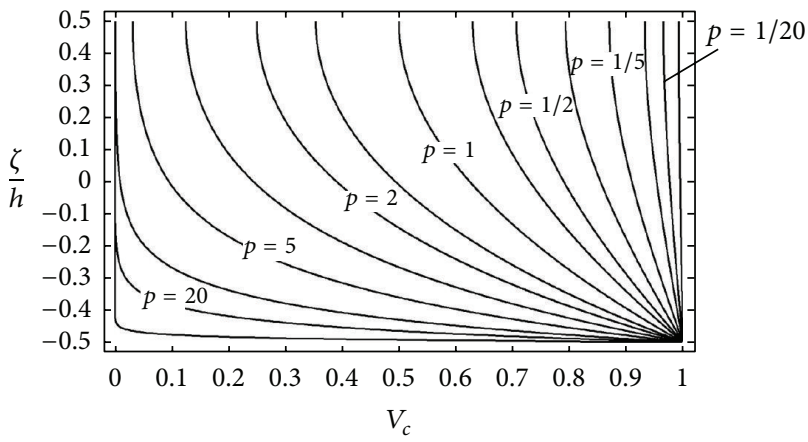

(e)

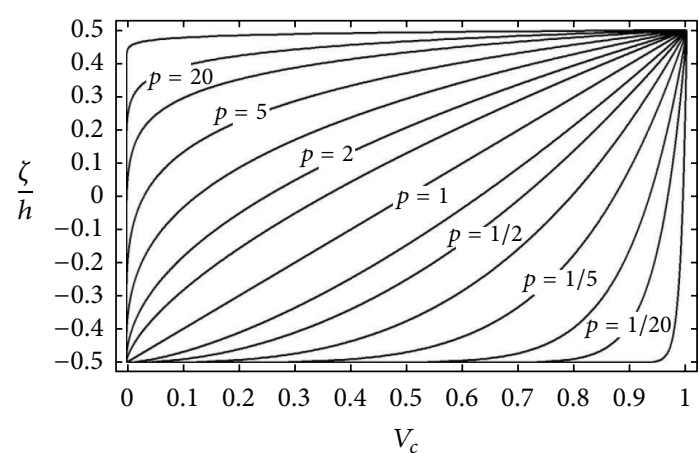

(b)

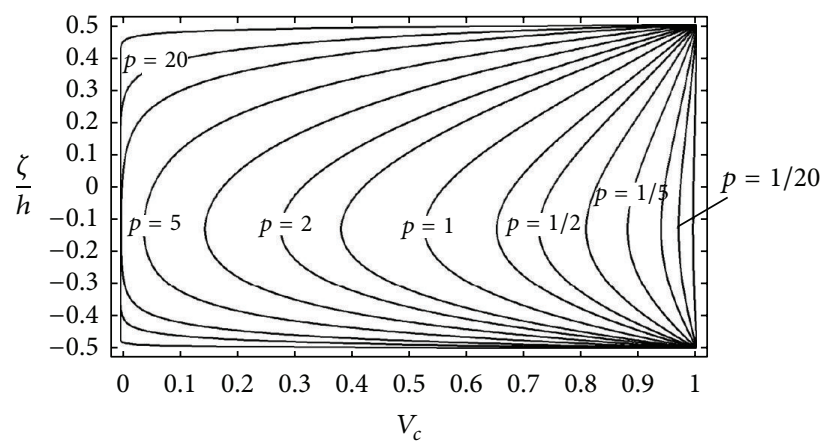

(d)

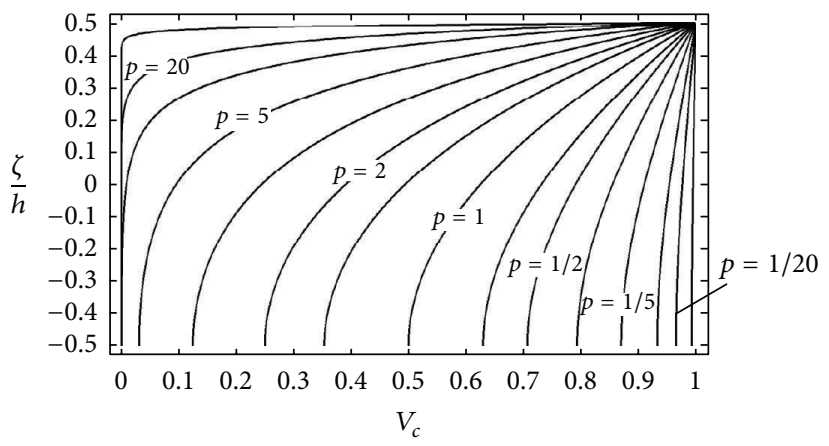

(f)

FIGURE 2: Variations of the ceramic volume fraction $V_{C}$ through the thickness for different values of the three parameters $a^{(1)}, b^{(1)}$, and $c^{(1)}$ and the power-law index $p^{(1)}$ for a single-layered shell: (a) $\mathrm{FGM}_{1\left(a^{(1)}=1 / b^{(1)}=0 / c^{(1)} / p^{(1)}\right)}$, (b) $\mathrm{FGM}_{2\left(a^{(1)}=1 / b^{(1)}=0 / c^{(1)} / p^{(1)}\right)}, \quad(\mathrm{c})$ $\mathrm{FGM}_{1\left(a^{(1)}=1 / b^{(1)}=1 / c^{(1)}=2 / p^{(1)}\right)}$, (d) $\mathrm{FGM}_{2\left(a^{(1)}=1 / b^{(1)}=1 / c^{(1)}=2 / p^{(1)}\right)}$, (e) $\mathrm{FGM}_{1(a=1 / b=0.5 / c=2 / p)}$, and (f) $\mathrm{FGM}_{2\left(a^{(1)}=1 / b^{(1)}=0.5 / c^{(1)}=2 / p^{(1)}\right)}$.

$$
\begin{aligned}
& N_{21}\left(\alpha_{1}, 0, t\right)+\frac{M_{21}\left(\alpha_{1}, 0, t\right)}{R_{1}} \\
& \quad=N_{21}\left(\alpha_{1}, 2 \pi, t\right)+\frac{M_{21}\left(\alpha_{1}, 2 \pi, t\right)}{R_{1}}, \\
& T_{2}\left(\alpha_{1}, 0, t\right)=T_{2}\left(\alpha_{1}, 2 \pi, t\right), \\
& M_{2}\left(\alpha_{1}, 0, t\right)=M_{2}\left(\alpha_{1}, 2 \pi, t\right), \\
& M_{21}\left(\alpha_{1}, 0, t\right)=M_{21}\left(\alpha_{1}, 2 \pi, t\right), \\
& \quad \alpha_{1}^{0} \leq \alpha_{1} \leq \alpha_{1}^{1} .
\end{aligned}
$$

In analogous way, in order to consider a toroidal shell of revolution it is necessary to implement the kinematic and physical compatibility conditions between the two computational parallels with $\alpha_{1}^{0}=0$ and with $\alpha_{1}^{1}=2 \pi$ :

Kinematic compatibility conditions along the closing parallel $\left(\alpha_{1}=0,2 \pi\right)$

$$
\begin{array}{ll}
u_{1}\left(0, \alpha_{2}, t\right)=u_{1}\left(2 \pi, \alpha_{2}, t\right), & u_{2}\left(0, \alpha_{2}, t\right)=u_{2}\left(2 \pi, \alpha_{2}, t\right), \\
u_{3}\left(0, \alpha_{2}, t\right)=u_{3}\left(2 \pi, \alpha_{2}, t\right), & \\
\beta_{1}\left(0, \alpha_{2}, t\right)=\beta_{1}\left(2 \pi, \alpha_{2}, t\right), & \beta_{2}\left(0, \alpha_{2}, t\right)=\beta_{2}\left(2 \pi, \alpha_{2}, t\right), \\
& \alpha_{2}^{0} \leq \alpha_{2} \leq \alpha_{2}^{1},
\end{array}
$$




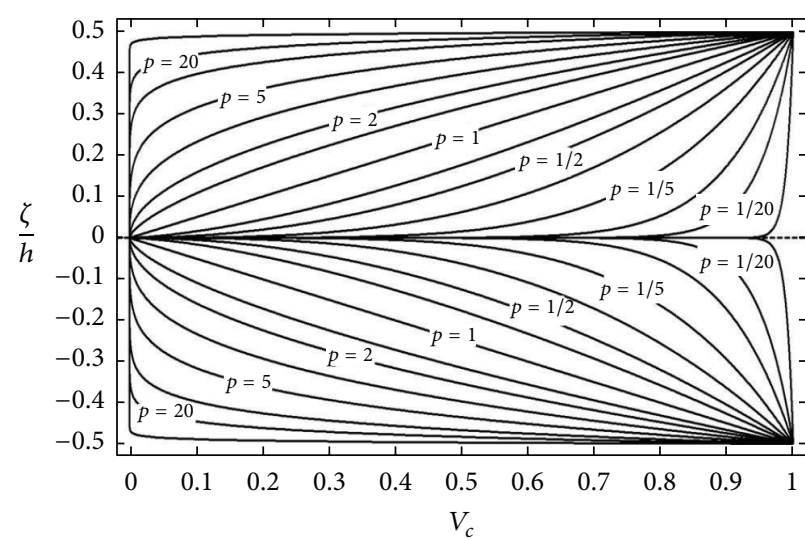

(a)

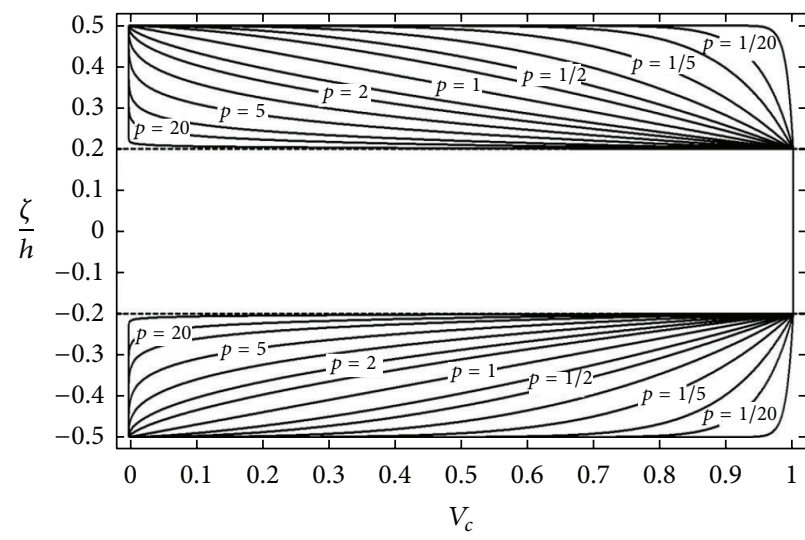

(c)

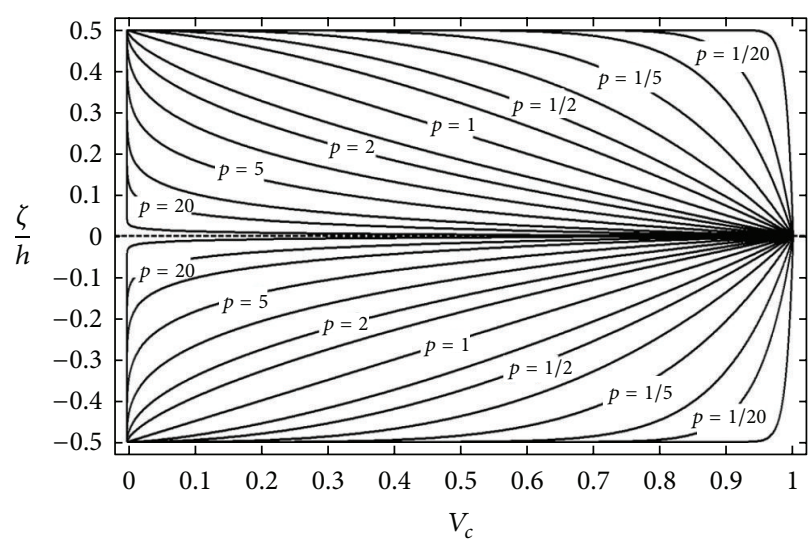

(b)

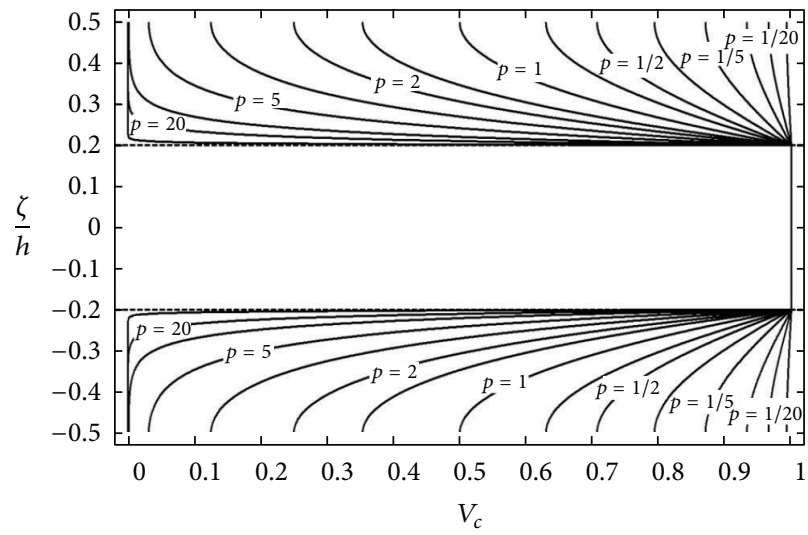

(d)

Figure 3: Variations of the ceramic volume fraction $V_{C}$ through the thickness for two-layered and three-layered laminated shells for

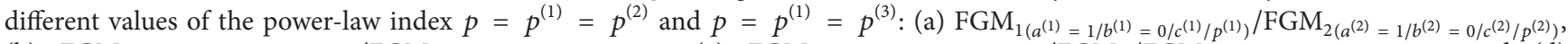

(b) $\quad \mathrm{FGM}_{2\left(a^{(1)}=1 / b^{(1)}=0 / c^{(1)} / p^{(1)}\right)} / \mathrm{FGM}_{1\left(a^{(2)}=1 / b^{(2)}=0 / c^{(2)} / p^{(2)}\right)}$, (c) $\mathrm{FGM}_{2\left(a^{(1)}=1 / b^{(1)}=0 / c^{(1)} / p^{(1)}\right)} / \mathrm{FGM}_{C} / \mathrm{FGM}_{1\left(a^{(3)}=1 / b^{(3)}=0 / c^{(3)} / p^{(3)}\right)}$, and $(\mathrm{d})$ $\mathrm{FGM}_{2\left(a^{(1)}=1 / b^{(1)}=0.5 / c^{(1)}=2 / p^{(1)}\right)} / \mathrm{FGM}_{\mathrm{C}} / \mathrm{FGM}_{1\left(a^{(3)}=1 / b^{(3)}=0.5 / c^{(3)}=2 / p^{(3)}\right)}$.

Physical compatibility conditions along the closing parallel $\left(\alpha_{1}=0,2 \pi\right)$

$$
\begin{aligned}
& N_{1}\left(0, \alpha_{2}, t\right)+\frac{M_{1}\left(0, \alpha_{2}, t\right)}{R_{1}} \\
& =N_{1}\left(2 \pi, \alpha_{2}, t\right)+\frac{M_{1}\left(2 \pi, \alpha_{2}, t\right)}{R_{1}}, \\
& N_{12}\left(0, \alpha_{2}, t\right)+\frac{M_{12}\left(0, \alpha_{2}, t\right)}{R_{2}} \\
& \quad=N_{12}\left(2 \pi, \alpha_{2}, t\right)+\frac{M_{12}\left(2 \pi, \alpha_{2}, t\right)}{R_{2}}, \\
& T_{1}\left(0, \alpha_{2}, t\right)=T_{1}\left(2 \pi, \alpha_{2}, t\right), \\
& M_{1}\left(0, \alpha_{2}, t\right)=M_{1}\left(2 \pi, \alpha_{2}, t\right), \\
& M_{12}\left(0, \alpha_{2}, t\right)=M_{12}\left(2 \pi, \alpha_{2}, t\right) \\
& \alpha_{2}^{0} \leq \alpha_{2} \leq \alpha_{2}^{1} .
\end{aligned}
$$

\section{Discretized Equations and Numerical Implementation}

The Generalized Differential Quadrature method is used to discretize the spatial derivatives in the governing equations in terms of generalized displacements, as well as boundary conditions (see Tornabene [49] for a brief review). Throughout the paper, the Chebyshev-Gauss-Lobatto (C-G-L) grid distribution is assumed, for which the coordinates of grid points $\left(\alpha_{1 i}, \alpha_{2 j}\right)$ along the reference surface are in the discrete form:

$$
\begin{array}{r}
\alpha_{1 i}=\left(1-\cos \left(\frac{i-1}{N-1} \pi\right)\right) \frac{\left(\alpha_{1}^{1}-\alpha_{1}^{0}\right)}{2}+\alpha_{1}^{0}, \\
i=1,2, \ldots, N, \quad \text { for } \alpha_{1} \in\left[\alpha_{1}^{0}, \alpha_{1}^{1}\right], \\
\alpha_{2 j}=\left(1-\cos \left(\frac{j-1}{M-1} \pi\right)\right) \frac{\left(\alpha_{2}^{1}-\alpha_{2}^{0}\right)}{2}+\alpha_{2}^{0}, \\
j=1,2, \ldots, M, \quad \text { for } \alpha_{2} \in\left[\alpha_{2}^{0}, \alpha_{2}^{1}\right],
\end{array}
$$




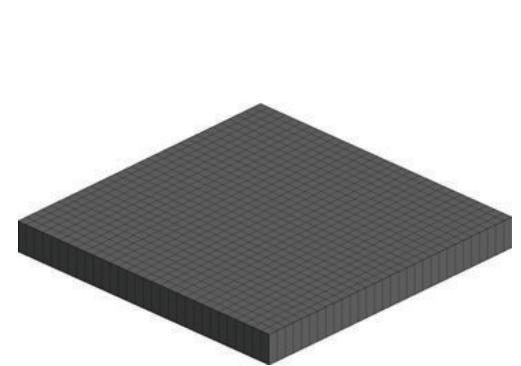

(a)

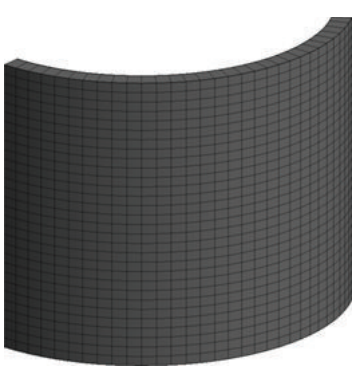

(b)

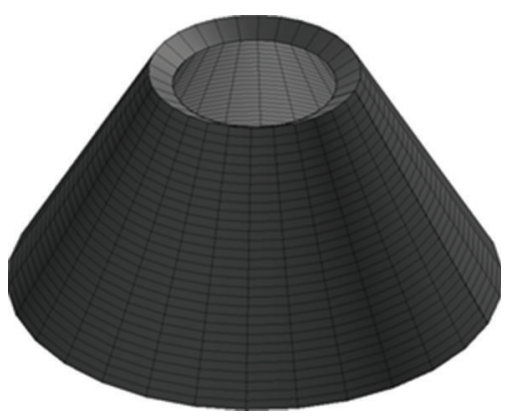

(c)

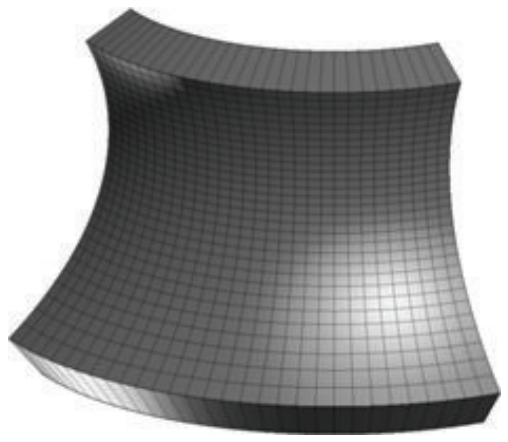

(e)

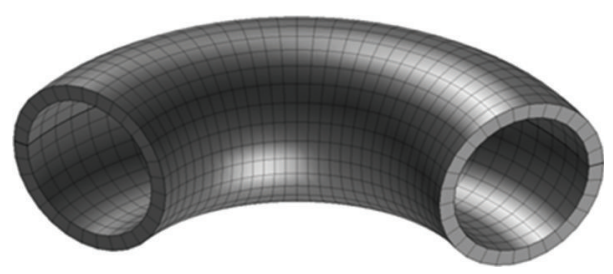

(d)

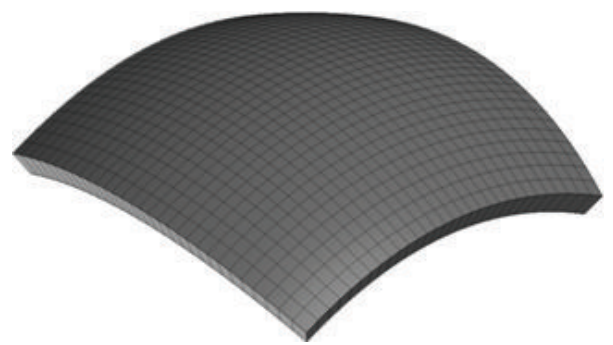

(f)

FIGURE 4: Six different types of FGM shells and panels: (a) rectangular plate (degenerate panel), (b) cylindrical panel (singly curved panel), (c) conical shell (singly curved shell), (d) toroidal shell panel (doubly curved shell panel of revolution), (e) catenoidal panel (doubly curved panel of revolution), and (f) elliptic paraboloid (completely doubly curved panel).

where $N, M$ are the total number of sampling points used to discretize the domain in $\alpha_{1}$ and $\alpha_{2}$ directions, respectively, of the doubly curved shell. It has been proven that, for the Lagrange interpolating polynomials, the Chebyshev-GaussLobatto sampling points rule guarantees convergence and efficiency to the GDQ technique [23, 43-45, 67, 68]. For the static analysis, when the inertias (21) are set to zero, the GDQ procedure enables to write the governing equations (20) and the boundary and compatibility conditions (22)(29) in discrete form, transforming each space derivative into a weighted sum of node values of independent variables using the Differential Quadrature rule [26, 49]:

$$
\left.\frac{\partial^{n} f(x)}{\partial x^{n}}\right|_{x=x_{m}}=\sum_{k=1}^{T} \varsigma_{m k}^{(n)} f\left(x_{k}\right), \quad m=1,2, \ldots, T .
$$

Each approximate equation is valid in a single sampling point. Thus, the whole system of differential equations has been discretized and the global assembling leads to the following set of linear algebraic equations:

$$
\left[\begin{array}{l|l}
\mathbf{K}_{b b} & \mathbf{K}_{b d} \\
\hline \mathbf{K}_{d b} & \mathbf{K}_{d d}
\end{array}\right]\left[\begin{array}{l}
\boldsymbol{\delta}_{b} \\
\hline \boldsymbol{\delta}_{d}
\end{array}\right]=\left[\begin{array}{l}
\mathbf{f}_{b} \\
\hline \mathbf{f}_{d}
\end{array}\right] .
$$

In the above mentioned matrices and vectors, the partitioning is set forth by subscripts $b$ and $d$, referring to the system degrees of freedom and standing for boundary and domain, respectively. In this sense, $b$-equations represent the discrete boundary conditions, which are valid only for the points lying on the constrained edges of the shell, while $d$-equations are the equilibrium equations, assigned on the interior nodes. In order to make the computation more efficient, static condensation of nondomain degrees of freedom is performed:

$$
\left(\mathbf{K}_{d d}-\mathbf{K}_{d b} \mathbf{K}_{b b}^{-1} \mathbf{K}_{b d}\right) \boldsymbol{\delta}_{d}=\mathbf{f}_{d}-\mathbf{K}_{d b} \mathbf{K}_{b b}^{-1} \mathbf{f}_{b} .
$$

The deflection of the considered structures can be determined by solving the linear algebraic problem (33). In particular, the 

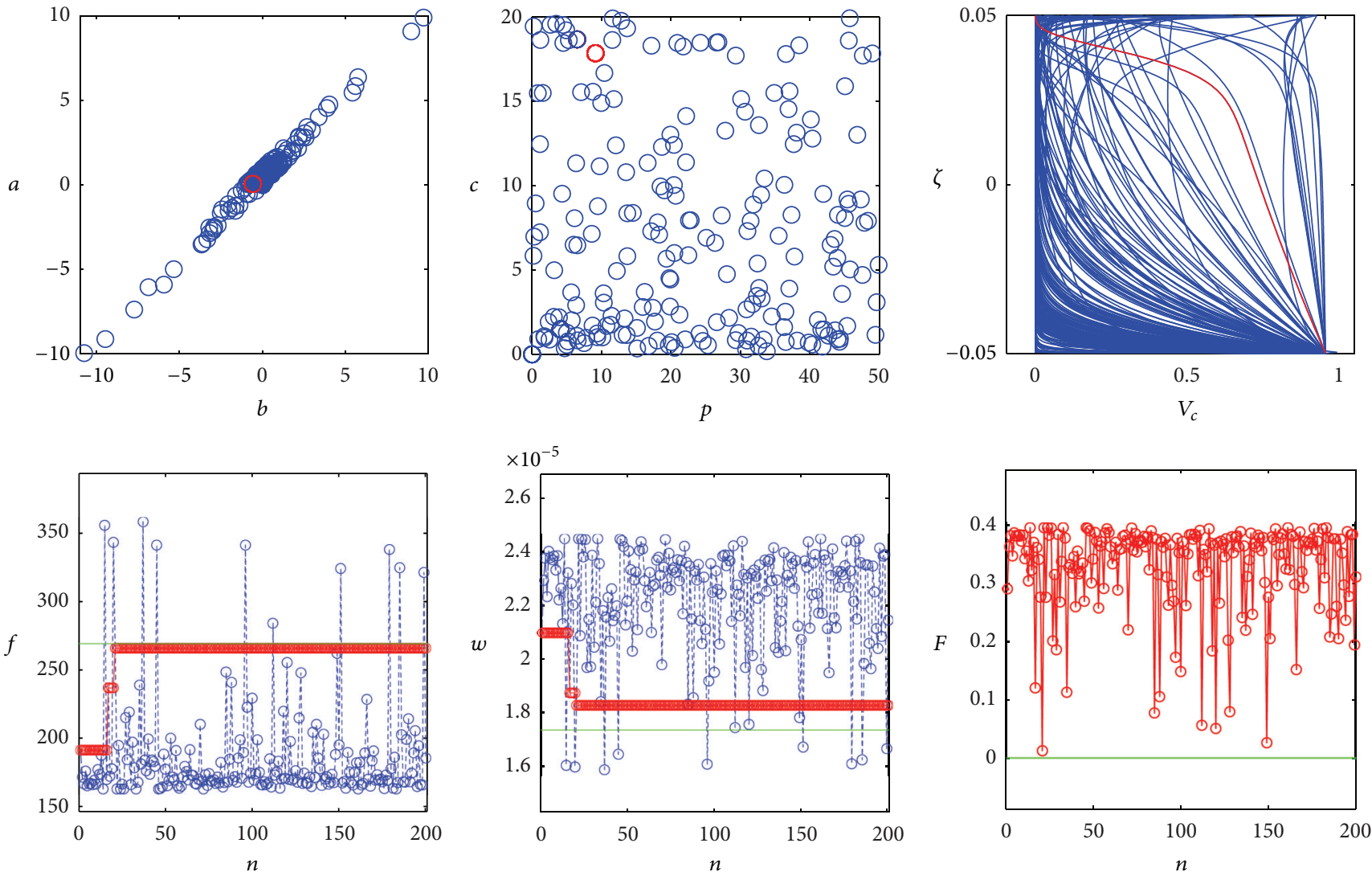

FIGURE 5: Square plate: Monte Carlo optimization with $\eta=1$; final optimized frequency $f=265.364 \mathrm{~Hz}$.

solution procedure by means of the GDQ technique has been implemented in a personal code.

Differently from the static case, when the external forces $q_{1}^{+}, q_{1}^{-}, q_{2}^{+}, q_{2}^{-}, q_{n}^{+}$, and $q_{n}^{-}(19)$ are set to zero, the free vibration of laminated composite doubly curved shells and panels can be studied. Using the method of variable separation, it is possible to seek solutions that are harmonic in time and whose frequency is $f=\omega / 2 \pi$. The generalized displacements can be written as follows:

$$
\begin{aligned}
& u_{1}\left(\alpha_{1}, \alpha_{2}, t\right)=U^{1}\left(\alpha_{1}, \alpha_{2}\right) e^{i \omega t}, \\
& u_{2}\left(\alpha_{1}, \alpha_{2}, t\right)=U^{2}\left(\alpha_{1}, \alpha_{2}\right) e^{i \omega t}, \\
& u_{3}\left(\alpha_{1}, \alpha_{2}, t\right)=U^{3}\left(\alpha_{1}, \alpha_{2}\right) e^{i \omega t}, \\
& \beta_{1}\left(\alpha_{1}, \alpha_{2}, t\right)=B^{1}\left(\alpha_{1}, \alpha_{2}\right) e^{i \omega t}, \\
& \beta_{2}\left(\alpha_{1}, \alpha_{2}, t\right)=B^{2}\left(\alpha_{1}, \alpha_{2}\right) e^{i \omega t},
\end{aligned}
$$

where the vibration spatial amplitude values $U^{1}, U^{2}, U^{3}, B^{1}$, and $B^{2}$ fulfil the fundamental differential system (20). Each approximate equation is valid in a single sampling point. Thus, the whole system of differential equations can be discretized and the global assembling leads to a set of linear eigenvalue problem. When kinematic condensation of nondomain degrees of freedom is performed, one gets

$$
\left(\mathbf{K}_{d d}-\mathbf{K}_{d b}\left(\mathbf{K}_{b b}\right)^{-1} \mathbf{K}_{b d}\right) \boldsymbol{\delta}_{d}=\omega^{2} \mathbf{M}_{d d} \boldsymbol{\delta}_{d} .
$$

The natural frequencies of the structure $f_{r}=\omega_{r} / 2 \pi$, for $r=1,2, \ldots, 5(N-2) \times(M-2)$, can be determined by solving the standard eigenvalue problem (35). In particular, the solution procedure by means of the GDQ technique has been implemented in a personal code. The above partitioning (35) is set forth by subscripts $b$ and $d$, referring to the system degrees of freedom and standing for boundary and domain, respectively. Finally, the results in terms of frequencies are obtained using an eigenvalue function. With the present approach, differing from the finite element method, no integration occurs prior to the global assembly of the linear system, and this represents a further computational cost saving in favour of the Differential Quadrature technique.

\section{Optimization Algorithms}

4.1. Genetic Algorithm (GA) Optimization Method. The GA approach to optimization was probably introduced at first by Holland [107], while a comprehensive reference including implementation procedures and application notes can be found in later work, like, for instance, the seminal book by Goldberg [99]. GA tries to implement and imitate in 

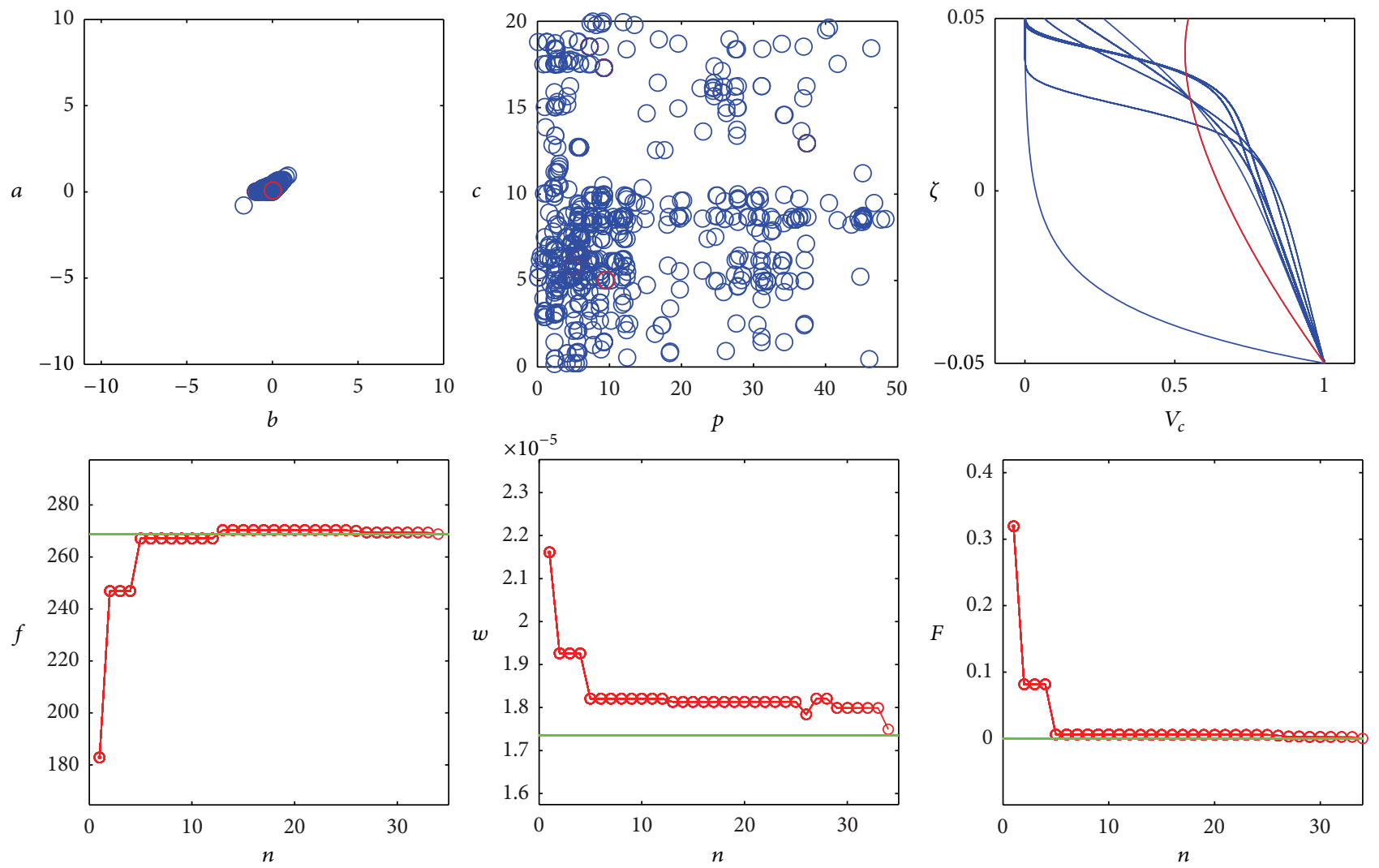

FIGURE 6: Square plate: Genetic Algorithm optimization with $\eta=1$; final optimized frequency $f=268.816 \mathrm{~Hz}$.

a mathematical framework the law of evolution, which Darwin introduced to explain the changes in nature towards individuals better suited to the environment in which they live: GA can be in fact classified as a typical populationbased optimization algorithm. Generation by generation, populations evolve improving the fitness function which represents the ability to survive in a defined environment; the individuals less adapting to the surrounding environment do not mate and their genetic set of chromosomes is lost. A solution is represented by a chromosome, constituted by a set of genes representing the parameters of the solution. The mathematical implementation follows with the coding of a chromosome in binary and with the application of some computational functions like mutations, crossover, and elitarism. When elitarism is set, some of the best individuals are replicated in the following generation without any change in a perfect replication from father to son. The concept of crossover implies a change between genes of two solutions and imitates the reproduction in which the son possesses a part of genes from his mother and the remaining from the father. Aim of mutations, as it happens in nature, is to randomly change some genes of the individual to test new configurations: from an optimization point of view, mutations help in exploring new zones of the space and are useful to avoid the problem of "local minimum" capturing.
According to the work by Konak et al. [106], the fitness is the main driver of the capability to survive and to pass genes to the next generation: the chromosome is in fact decoded from binary to decimal and tested in the fitness only after the application of mating functions requiring a binary coding. Modern approaches to the application of GA lie in new formulations and in the introduction of hybridization with other optimization strategies [108]; also Pareto-based analysis for multiobjective optimization [103] has been evaluated, and improvement of GA by the application of fuzzy sets and neural networks [109] has been proposed to solve complex tasks. The algorithm implemented in this work follows the procedure proposed by Goldberg [99], and the fitness has been defined by authors considering the closeness of the solution found to the value set by the designer and the feasibility of the volume fraction distribution in the thickness $\left(0<V_{C}^{(k)}<1\right)$. The pseudocode of the GA implemented is shown in the following Pseudocode 1.

4.2. Monte Carlo (MC) Optimization Method. The Monte Carlo technique has been developed to support early studies related to the nuclear physics; the idea is to find the best approximation of a constant or to solve a problem through a statistical way, obtaining the results from a very large set 

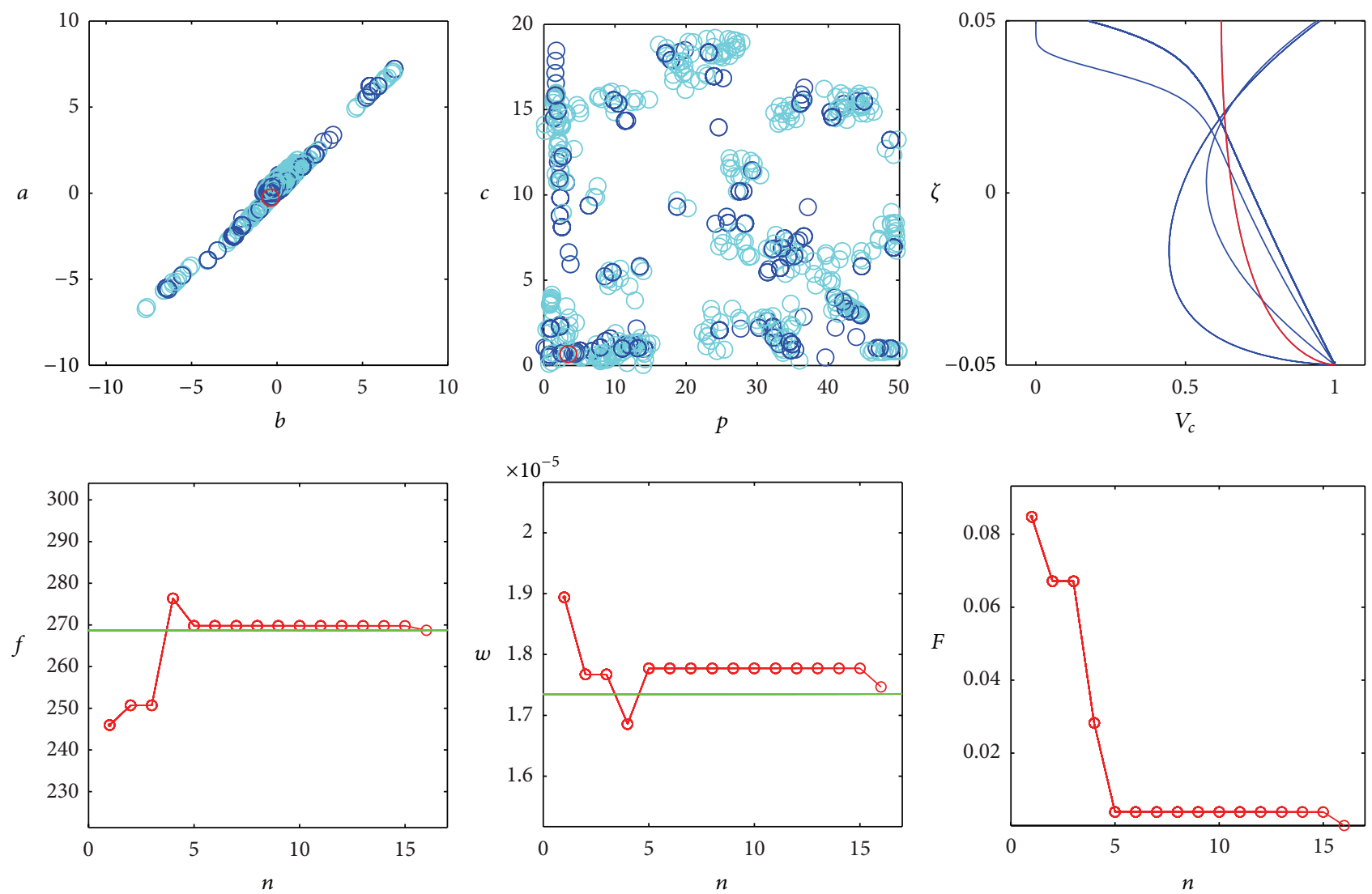

FIGURE 7: Square plate: Particle Swarm Optimization with $\eta=1$; final optimized frequency $f=268.710 \mathrm{~Hz}$.

of random inputs. From a mathematical and formal point of view, for instance, the paper by Mosegaard and Sambridge [123] introduces the way by which an integral in the form

$$
I=\int_{X} g(x) f(x) d x
$$

can be evaluated by the generation of random samples $x_{1}, x_{2}, x_{3}, x_{4}, \ldots, x_{S}$ of $x$, when $f(x)$ is an appropriate probability distribution and $g(x)$ is the function of which the integral have to be computed. The integral can be so computed by the expression

$$
I \approx \frac{1}{S} \sum_{s=1}^{S} g\left(x_{s}\right) .
$$

The MC methods are very simple, but studies are focused on the probability distribution shapes providing best results and on the software methods to generate random numbers; this is not a trivial issue, since it can be complex to generate a set of random numbers which are not dependent on the clock of the processor or on other hardware timers. Obviously, considering computational efforts, the MC method is very time expensive; however, due to the increasing computations capabilities of personal computers and the improving of the capabilities which are a constant trend in years, this approach has been recently reconsidered and still applied to a wide range of applications. The continuous improvement in randomizer algorithms helps in the gain of good performances, which are often obtained exploiting the capabilities of clusters of computers. The main critical aspect related to the MC methods application in engineering is that it requires a lot of iteration to obtain a solution; following this approach, in fact, the space is explored in a completely random way and no attention is focused on zones in which solutions are better than the average. The literature presents a lot of interesting applications of $\mathrm{MC}$ to engineering problems, like the works [124-126] show; MC is also widely applied to games and strategy since it allows keeping in consideration all the possible scenarios evolving from a situation: some papers dealing with this issue can be found in [127]. A pseudocode of the MC algorithm is included in Pseudocode 2.

4.3. Particle Swarm Optimization (PSO) Method. The PSO algorithm used in this paper is similar to the one proposed by Birge [115], with some variations due to the particular application to the FGM optimization problem. According to the general implementation of PSO, the position of a particle in the $n$-dimensional solution space can be considered a 

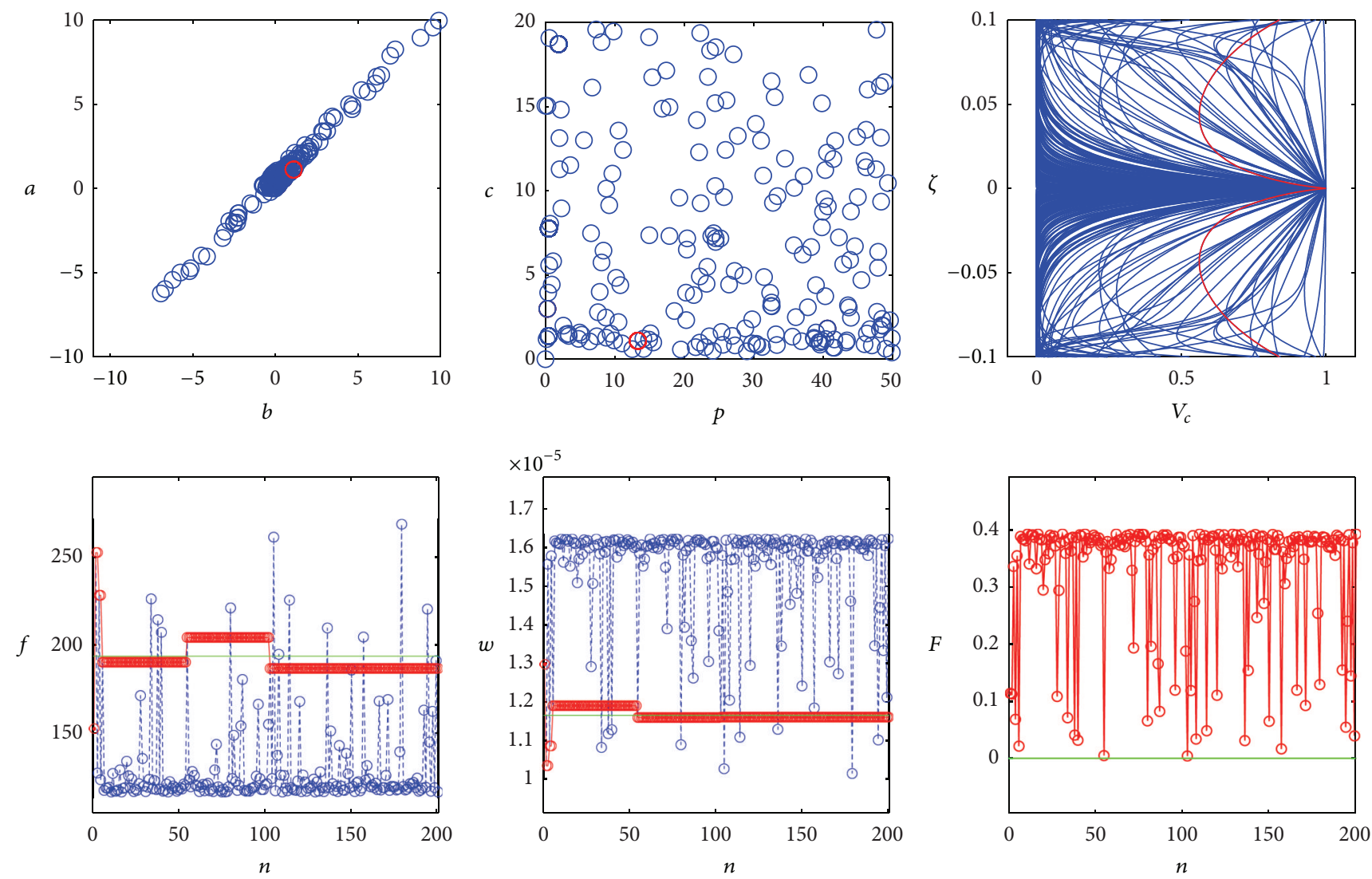

FIgURE 8: Cylindrical panel: Monte Carlo optimization with $\eta=1$; final optimized displacement $w=1.1596 \cdot 10^{-5} \mathrm{~m}$.

solution to the problem in the $n$-dimensional space of the parameters; the $n$-dimensional speed which is computed in the algorithm represents the direction towards a new position in the $n$-dimensional space. The velocity can be obtained by

$v_{i}^{(m)}=\phi^{(m)} v_{i}^{(m-1)}+c_{1} a_{1}\left(P_{b i}-P_{i}^{(m-1)}\right)+c_{2} a_{2}\left(P_{b G_{b}}-P_{i}^{(m-1)}\right)$,

where $m$ is the algorithm step ( $n_{G}$ generations); $i$ is the index of parameter of the single particle; $\phi^{(m)}$ is the inertia function; $v_{i}^{(m)}$ is the velocity of the $i$ th particle at the $m$-step; $P_{b i}$ is the best position found by the $i$ th particle; $G_{b}$ is the global best position (it is the best position found by the whole swarm); $a_{1}, a_{2}$ are the acceleration constants; $c_{1}, c_{2}$ are random numbers in the interval $[0,1]$.

The following formulation can be applied to obtain the new position:

$$
P_{i}^{(m)}=P_{i}^{(m-1)}+v_{i}^{(m)},
$$

where $P_{i}^{(m)}$ is the position of the $i$ th particle at the $m$-step and $v_{i}^{(m)}$ is the velocity of the $i$ th particle at the $m$-step. One of the problems of the FGM profile shape in the thickness is that a small variation in $a^{(k)}, b^{(k)}, c^{(k)}$, and $p^{(k)}$ power-law parameters can lead to a solution showing a volume fraction inconsistent $\left(V_{C}^{(k)}<0\right.$ or $\left.V_{C}^{(k)}>1\right)$. The PSO algorithm, in fact, belongs to the so-called "trajectory based methods" and sweeps the space following a path in which the new position is equal to the previous one plus a constant segment (the speed). For this reason, sometimes the updated position of a particle lies in a zone in which the volume fraction is out of limit; in this case, the classical implementation of the algorithm stops since the trajectory enters in a trap from which it is impossible to escape. The new position, in fact, can lead to inconsistent volume fractions and the algorithm stalls. In order to solve this problem, a check has been introduced in the algorithm (step 11 and step 12 in Pseudocode 3), so that if an unfeasible position is found by the algorithm, the velocity is rejected and changed until a new valid position is found. By this way, a forecast of the new position is computed and the PSO velocity is accepted if leading to a valid solution, randomized if it does not. The end criterion is due to one of these conditions: the achievement of the maximum number of iterations, or a condition in which after $n_{\varepsilon}$ generations the solution does not improve. The PSO seems to be very attractive for optimization since the studies by $\mathrm{Hu}$ et al. [116] and by Ceruti et al. [102] present advantages with respect to GA: easiness of software code implementation, need for the definition of few setting 

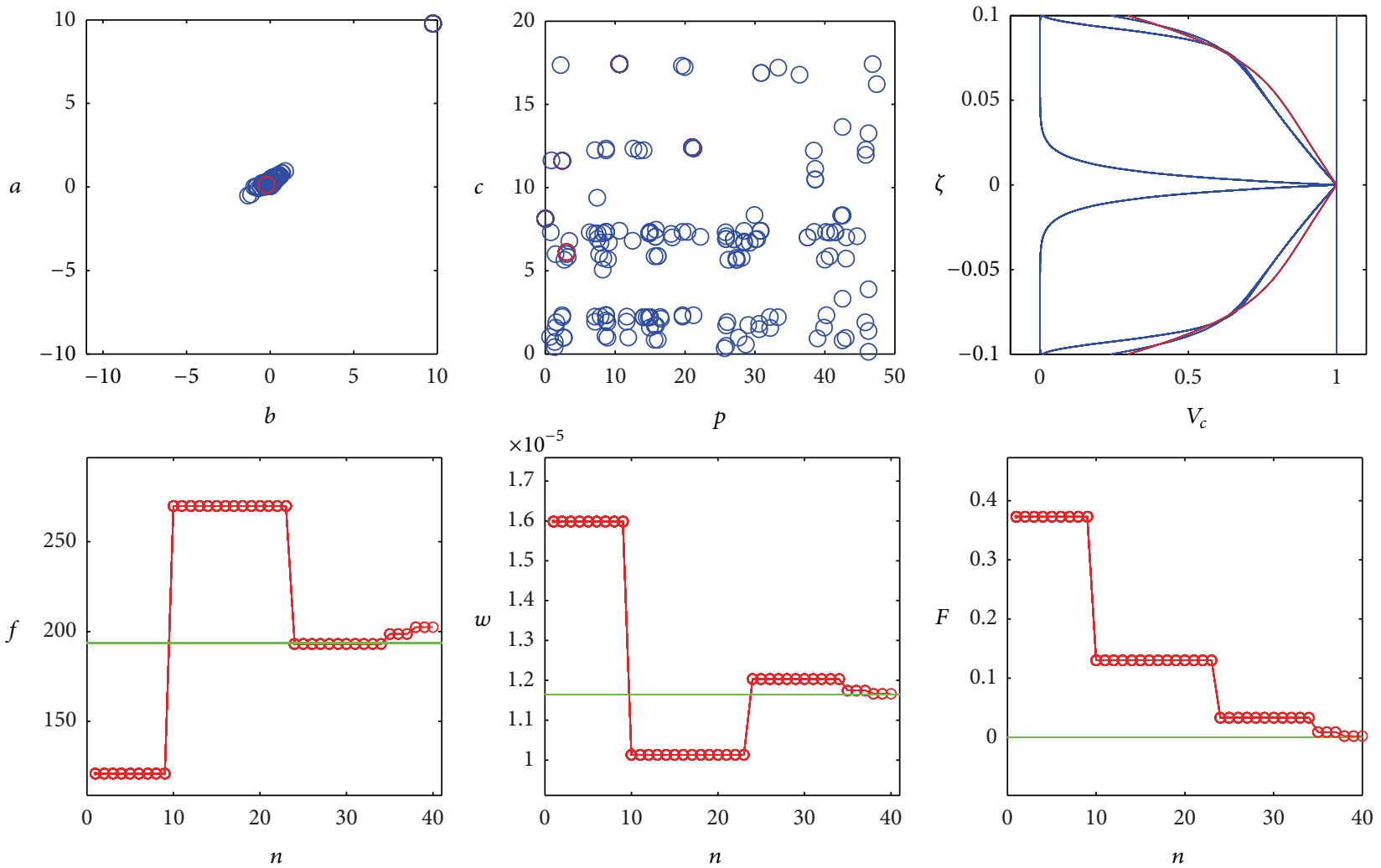

FIgURE 9: Cylindrical panel: Genetic Algorithm optimization with $\eta=1$; final optimized displacement $w=1.1668 \cdot 10^{-5} \mathrm{~m}$.
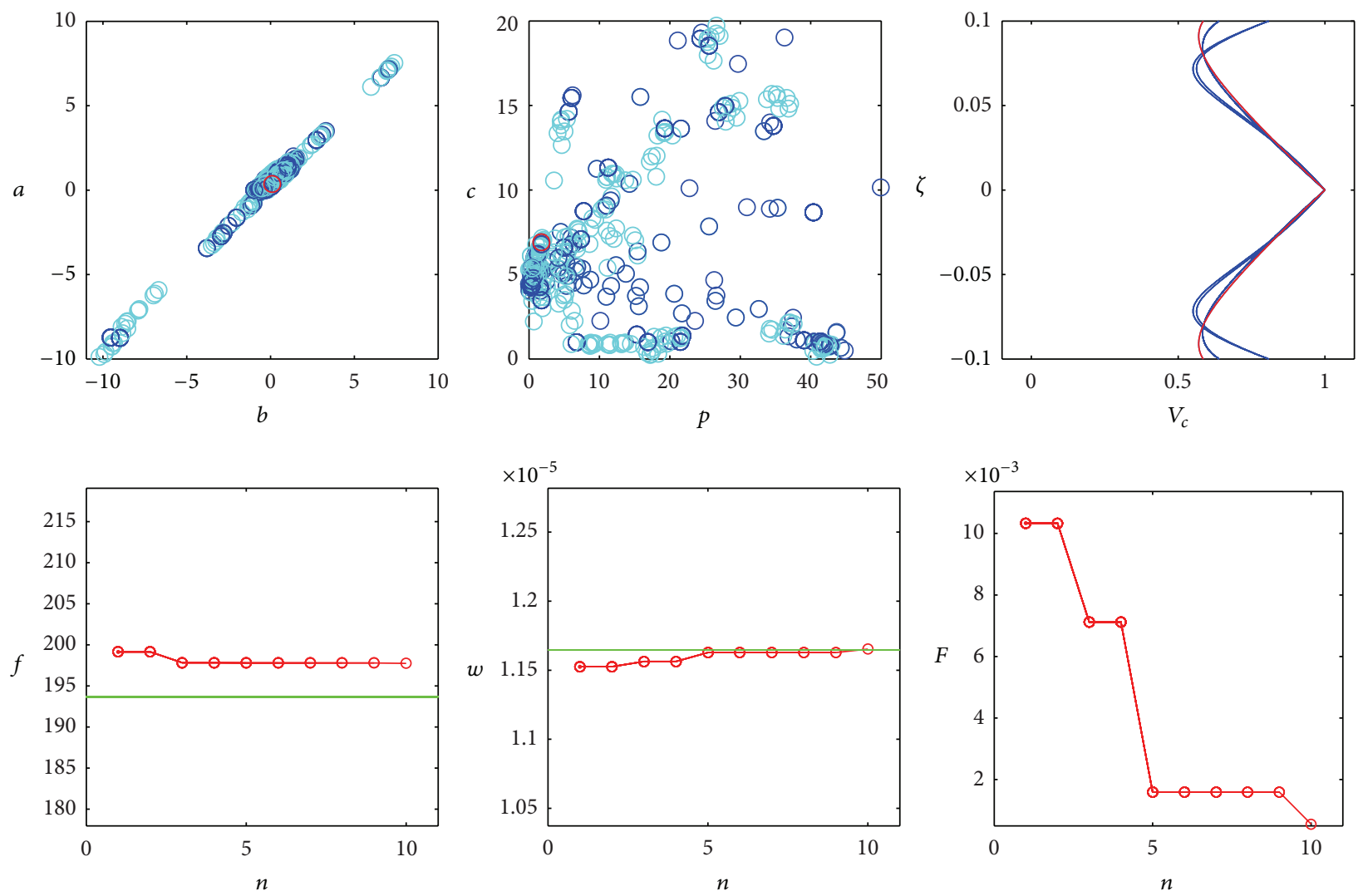

Figure 10: Cylindrical panel: Particle Swarm Optimization with $\eta=1$; final optimized displacement $w=1.1653 \cdot 10^{-5} \mathrm{~m}$. 

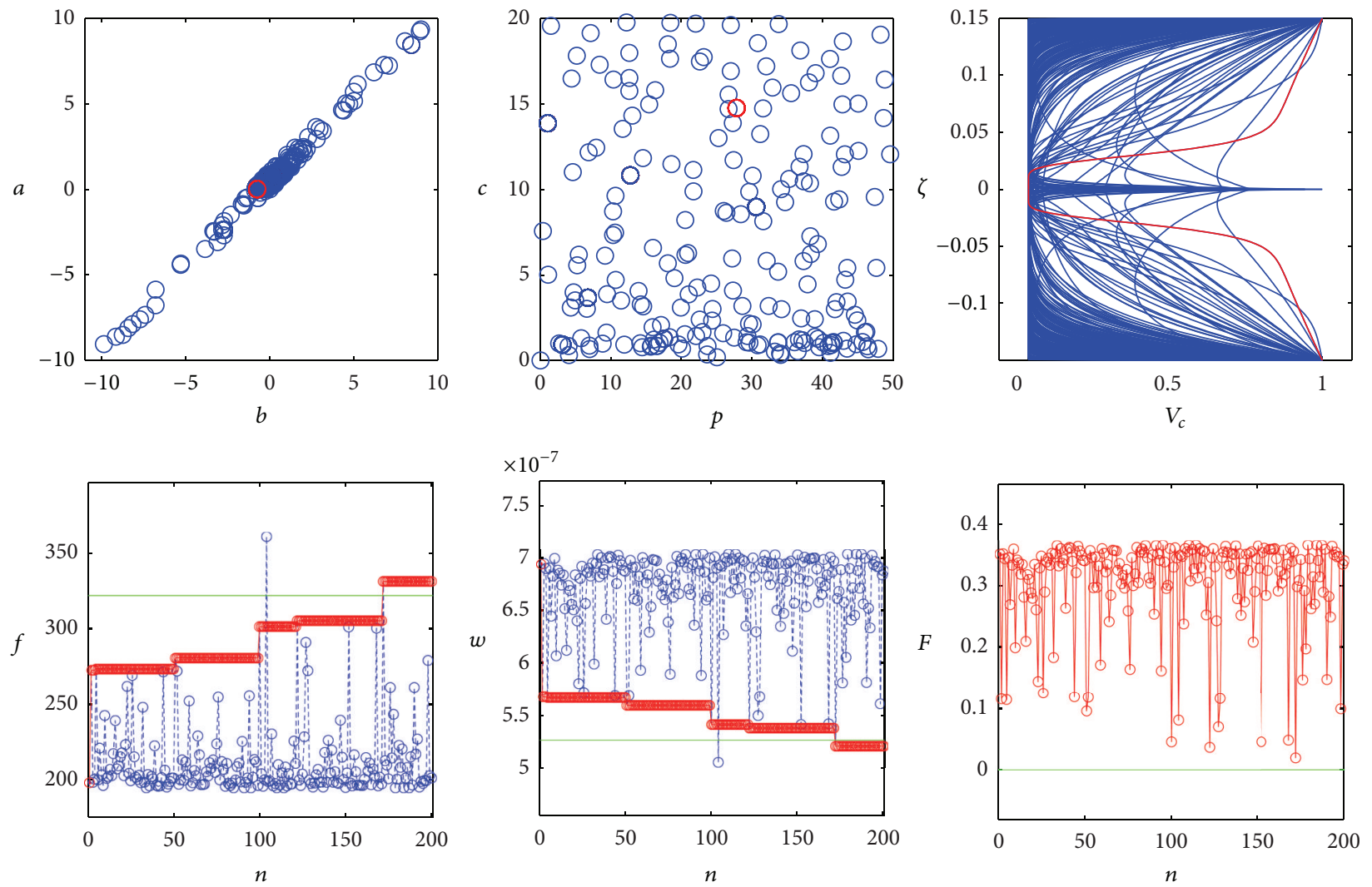

FIGURE 11: Conical shell: Monte Carlo optimization with $\eta=0.5$; final optimized frequency $f=330.946 \mathrm{~Hz}$ and final optimized displacement $w=5.2073 \cdot 10^{-7} \mathrm{~m}$.

parameters, and good fitting to engineering application. The PSO implementation and the modifications introduced for this application are described in Pseudocode 3, where an illustrative pseudocode is presented.

\section{Numerical Applications and Results}

In the present section, some results and considerations about the mixed static and dynamic optimization problem of fourparameter functionally graded doubly curved and degenerate shells and panels are presented. The analysis has been carried out by means of numerical procedures illustrated above. Different types of structures are considered in the present paper. One of the aims of this study is to show some numerical examples about flat plates, singly curved, and doubly curved shells and panels made of FGMs. The six considered structures are depicted in Figure 4. In order to describe the middle surface of the given structures, theoretical formulae of differential geometry $[7,23,43,74,78]$ are used. The mathematical development of the differential geometry applied to doubly curved shells was deeply explained in $[23,43,74,78]$. So, in the following, only a few formulae are reported. Furthermore, it is worthwhile noting that the GDQ procedure enables to evaluate the parameters concerning the shell geometry as reported in $[74,78]$. For all the GDQ results presented below, the Chebyshev-Gauss-Lobatto grid distributions (30) with $N=M=31$ along the reference surface have been assumed, and the geometrical parameters with their derivatives are numerically evaluated using the GDQ method [26]. The FGM nomenclature used in the present work is identified by the same convention presented in the previous work by Tornabene et al. [70]. In an analogous way, the geometrical boundary conditions are defined considering similar convention used in the previous works [23, 43, 44, 47, 49-53, 68-76] for shell and panel structures.

For a rectangular flat plate, the position vector $[74,78]$ can be written as

$$
\mathbf{r}\left(\alpha_{1}, \alpha_{2}\right)=-\alpha_{2} \mathbf{e}_{2}+\alpha_{1} \mathbf{e}_{3}
$$

In Figure 4(a) the considered square plate has the sides $a=$ $b=1 \mathrm{~m}$, thickness $h=0.1 \mathrm{~m}$, and it is subjected to a normal load $q_{n}^{+}=-10000 \mathrm{~Pa}$ at the top surface. The plate is free on two adjacent sides and clamped on the other two. The boundary conditions are indicated by the current nomenclature as CCFF. The square plate is a single-layered structure with $\mathrm{FGM}_{1\left(a^{(1)} / b^{(1)} / c^{(1)} / p^{(1)}\right)}$ four-parameter powerlaw distribution. In this case, the four parameters $a=a^{(1)}, b=$ $b^{(1)}, c=c^{(1)}$, and $p=p^{(1)}$ have to be determined by the optimization procedure.

The position vector for the cylindrical panel and conical shell of Figures 4(b) and 4(c) can be obtained from the conical shell formula, already presented in $[74,78]$ and reported here 

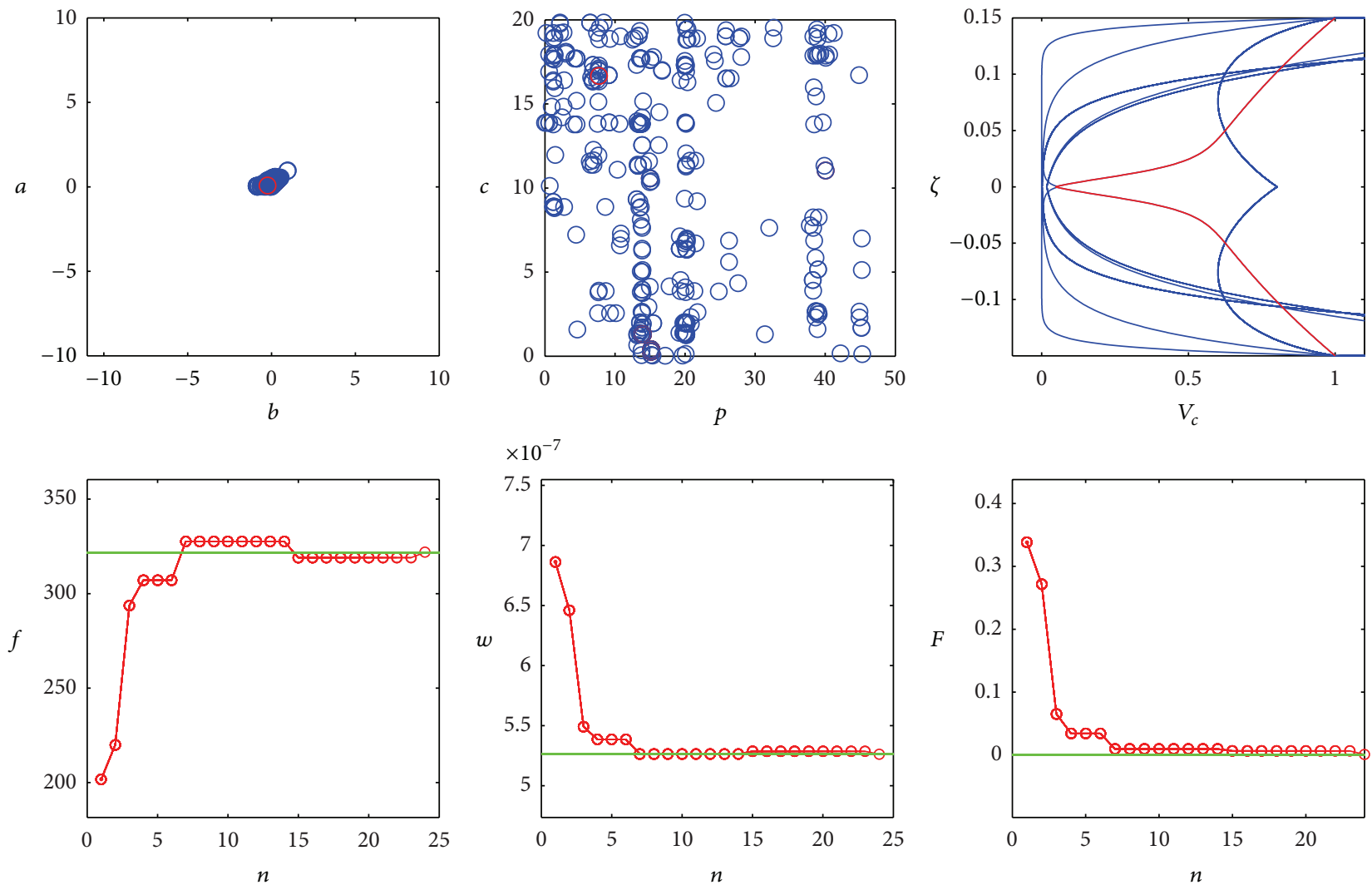

Figure 12: Conical shell: Genetic Algorithm optimization with $\eta=0.5$; final optimized frequency $f=321.890 \mathrm{~Hz}$ and final optimized displacement $w=5.2624 \cdot 10^{-7} \mathrm{~m}$.

Step (1) Set a max and min value for each gene

Step (2) Set the max number of generations $(T)$, the convergence tolerance $(\varepsilon)$ and the number of consecutive no improvements loops $\left(n_{\varepsilon}\right)$ after which algorithm ends, the number of genes $\left(n_{g}\right)$, and the number of population $\left(n_{\text {pop }}\right)$ members

Step (3) Initialize the first generation of population $(P)$ by randomly set $n_{\text {pop }}$ chromosomes, each one made by $n_{g}$ genes

Step (4) while $(i<T)$ or ( $\varepsilon$ for $n_{\varepsilon}$ loops false)

Step (5) Select $P^{\prime} \subset P$ (mating pool), initialize $P^{\prime \prime}=0$ (set of children)

Step (6) for $j=1$ to $n$

Step (7) Randomly select individuals (chromosomes $x_{a}$ and $x_{b}$ ) from $P^{\prime}$

Step (8) Obtain $x_{\text {child }}$ by applying crossover to $x_{a}$ and $x_{b}$ (with probability $p_{\text {cross }}$ )

Step (9) $\quad$ Mutate produced child $x_{\text {child }}$ to $x_{a}$ and $x_{b}$ (with probability $p_{\text {mut }}$ )

Step (10) Apply elitarism (if set)

Step (11) Update population $P^{\prime \prime}=P^{\prime} \cup x_{\text {child }}$

Step (12) end for

Step (13) $\quad P=$ survival respect to the fitness* $\left(P^{\prime}, P^{\prime \prime}\right)$

Step (14) end while

Step (15) best chromosome detection

${ }^{*}$ In this case the fitness is multiplied by a penalty term if one of the sets $\left[a^{(k)}, b^{(k)}, c^{(k)}, p^{(k)}\right]$ leads to volume fractions which are not feasible (e.g. percentage of one constituent in the thickness larger than 1 , less than 0 , imaginary number). 
Step (1) Set a max and min value for each parameter

Step (2) Set the max number of population $\left(n_{g}\right)$ members, the convergence tolerance $(\varepsilon)$ and the number of consecutive no improvements loops $\left(n_{\varepsilon}\right)$

Step (3) while $\left(i<n_{g}\right)$ or ( $\varepsilon$ for $n_{\varepsilon}$ loops false)

Step (4) Randomly generate a member of population $P_{i}$, following a probability

distribution (normal, exponential, Weibull [113]).

Step (5) Compute volume fraction distribution $\left(V_{C}\right)$ in the thickness for $P_{i}$

Step (6) $\quad$ if $0<V_{C}<1$

Step (7) $\quad i=i+1$

Step (8) Compute Fitness

Step (9) if Fitness $(i)>$ Best Fitness

Step (10) Best Fitness $=$ Fitness $(i)$

Step (11) Best member $=i$

Step (12) end if

Step (12) end if

Step (13) end while

Pseudocode 2: Monte Carlo pseudocode.

Step (1) Set number of particles $\left(n_{P}\right)$, number of parameters for each particle $\left(n_{\text {param }}\right)$, max number of generations $\left(n_{G}\right)$, convergence tolerance $(\varepsilon)$ and the number of consecutive no improvements loops $\left(n_{\varepsilon}\right), \delta$ (ratio between position and speed)

Step (2) Set particles boundary values: $\max \left(1: n_{\text {param }}\right), \min \left(1: n_{\text {param }}\right)$

Step (3) Randomly generate particles position $\left(P_{i}^{(0)}\right) \mid \min (i)<P_{i}^{(0)}<\max (i)$ and velocity $v_{i}^{(0)}$

Step (4) Evaluate fitness function $F_{i}=f\left(P_{i}^{(0)}\right), i=1, \ldots, n_{P}$

Step (5) Set $P_{b}=P^{(0)}, F_{\text {best }}=F, G_{b}=\min \left(F_{i}\right), g_{b}=$ index of the $\left(\min \left(F_{i}\right)\right)$ value

Step (6) for $m=1$ to $n_{G}$

Step (7) for $i=1$ to $n_{P}$

Step (8) $\quad \Delta v_{i}=c_{1} a_{1}\left(P_{b i}-P_{i}^{(m-1)}\right)+c_{2} a_{2}\left(P_{b G_{b}}-P_{i}^{(m-1)}\right)$

Step (9) $\quad v_{i}^{(m)}=\phi^{(m)} v^{(m-1)}+\Delta v_{i}$

Step (10) $\quad P_{i}^{(m)}=P_{i}^{(m-1)}+v_{i}^{(m)}$

Step (11) Compute Volume fraction distribution $\left(V_{C}\right)$ in the thickness for $P_{i}^{(m)}$

Step (12) while $\left(P_{i}^{(m)}>\max (i)\right)$ or $\left(P_{i}^{(m)}<\min (i)\right)$ or $\left(V_{C}<0\right)$ or $\left(V_{C}>1\right)$

Step (13) $\quad v_{i}^{(m)}=v^{(m-1)}+\delta \cdot(\max (i)-\min (i)) \cdot \operatorname{random}(0 \div 1)$

Step (14)

Step (15)

$P_{i}^{(m)}=P_{i}^{(m-1)}+v_{i}^{(m)}$

end while

Step (16) Evaluate fitness $F_{i}=f\left(P_{i}^{(m)}\right), i=1, \ldots, n_{P}$

Step (17) for $i=1, \ldots, n_{P}$

Step (18) if $F_{i}<F_{b i}$ then $P_{b i}=P_{i}^{(m)}$ and $F_{b i}=F_{i}$

Step (19) if $F_{i}<G_{b}$ then $G_{b}=F_{i}$ and $g_{b}=i$

Step (20) end for

Step (21) end for

Step (22) end for

Pseudocode 3: Particle Swarm Optimization pseudocode.

for the sake of completeness. The position vector for the conical shell can be written as follows:

$$
\begin{aligned}
\mathbf{r}\left(\alpha_{1}, \alpha_{2}\right)= & \left(R_{b}+\alpha_{1} \sin \alpha\right) \cos \alpha_{2} \mathbf{e}_{1} \\
& -\left(R_{b}+\alpha_{1} \sin \alpha\right) \sin \alpha_{2} \mathbf{e}_{2}+\alpha_{1} \cos \alpha \mathbf{e}_{3} .
\end{aligned}
$$

As it is well known [7, 74, 78], a conical shell [23, 42, 43, 49, 52 ] is a $2 \mathrm{D}$ structure having the middle surface generated by the rotation, about a fixed vertical axis, of an inclined straight line. The angle $\alpha$ is the top vertex angle of the cone, where the meridian angle $\varphi$ can be written as a function of it: $\varphi=$ $\pi / 2-\alpha$. Thus, for cylindrical panel, considering $\alpha=0$, the position vector (41) takes the form

$$
\mathbf{r}\left(\alpha_{1}, \alpha_{2}\right)=R_{b} \cos \alpha_{2} \mathbf{e}_{1}-R_{b} \sin \alpha_{2} \mathbf{e}_{2}+\alpha_{1} \mathbf{e}_{3} .
$$

The meridian abscissa $\alpha_{1}$ is defined as $\alpha_{1} \in[0, L]$, where $L$ is the length of the meridian. The presented parameters are graphically shown in the works $[23,42,43,49,52]$. The cylindrical panel (Figure $4(\mathrm{~b})$ ) is defined by $R=R_{b}=2 \mathrm{~m}$, 
TABLE 2: Optimization of the six different structures of Figure 4: (a) square plate, (b) cylindrical panel, (c) conical shell, (d) toroidal shell panel, (e) catenoidal panel, and (f) elliptic paraboloid. $\eta=1$ : optimization of the first frequency $f_{1} ; \eta=0$ : optimization of maximum bending displacement $w_{\max }=u_{3 \max } ; \eta=0.5$ : optimization of the first frequency $f_{1}$ and maximum bending displacement $w_{\max }=u_{3 \max }$.

\begin{tabular}{|c|c|c|c|c|c|c|c|c|}
\hline Method & $a$ & $b$ & $c$ & $p$ & Final frequency & Final displacement & Final fitness & Computational time (min) \\
\hline \multicolumn{9}{|c|}{ Rectangular plate } \\
\hline \multicolumn{9}{|c|}{$\eta=1:$ target frequency $f_{1 T}=268.753 \mathrm{~Hz}$} \\
\hline MC & 0.05582 & -0.56831 & 17.86455 & 9.14279 & 265.36499 & $1.8270 E-05$ & $1.2606 E-02$ & 25 \\
\hline GA & 0.08800 & -0.06160 & 5.02440 & 9.53080 & 268.81630 & $1.7493 E-05$ & $2.3583 E-04$ & 42 \\
\hline PSO & -0.25829 & -0.13011 & 0.67170 & 3.43176 & 268.71000 & $1.7466 E-05$ & $1.5970 E-04$ & 31 \\
\hline \multicolumn{9}{|c|}{ Cylindrical panel } \\
\hline \multicolumn{9}{|c|}{$\eta=0:$ target displacement $w_{\max T}=u_{3 \max T}=1.1646 \cdot 10^{-5} \mathrm{~m}$} \\
\hline MC & 1.11895 & 1.10588 & 1.09493 & 13.33798 & 186.78559 & $1.1596 E-05$ & $4.3053 E-03$ & 32 \\
\hline GA & 0.12710 & -0.33040 & 6.11930 & 3.03030 & 202.41150 & $1.1668 E-05$ & $1.8770 E-03$ & 14 \\
\hline PSO & 0.35190 & -0.26277 & 6.90518 & 1.75727 & 197.75230 & $1.1653 E-05$ & $5.8904 E-04$ & 20 \\
\hline \multicolumn{9}{|c|}{ Conical shell } \\
\hline \multicolumn{9}{|c|}{$\eta=0.5:$ target frequency $f_{1 T}=268.753 \mathrm{~Hz}$ and target displacement $w_{\max T}=u_{3 \max T}=5.2639 \cdot 10^{-7} \mathrm{~m}$} \\
\hline MC & 0.00984 & -0.74086 & 14.77422 & 27.83370 & 330.94622 & $5.2073 E-07$ & $1.9799 E-02$ & 35 \\
\hline GA & 0.08800 & -0.32650 & 16.65690 & 7.72240 & 321.89420 & $5.2624 E-07$ & $4.9477 E-04$ & 25 \\
\hline PSO & 0.05769 & -0.49331 & 9.85753 & 8.35549 & 322.04900 & $5.2615 E-07$ & $8.2088 E-04$ & 43 \\
\hline \multicolumn{9}{|c|}{ Toroidal shell panel } \\
\hline \multicolumn{9}{|c|}{$\eta=0:$ target displacement $w_{\max T}=u_{3 \max T}=3.9516 \cdot 10^{-5} \mathrm{~m}$} \\
\hline $\mathrm{MC}$ & 0.05350 & -0.54251 & 17.60320 & 20.64836 & 20.21863 & $3.9727 E-05$ & $5.3396 E-03$ & 21 \\
\hline GA & 0.02930 & -0.78200 & 12.72730 & 25.90420 & 20.56750 & $3.9495 E-05$ & $5.3143 E-04$ & 3 \\
\hline PSO & 0.10430 & -0.58284 & 8.53859 & 8.22430 & 20.48775 & $3.9519 E-05$ & $7.5919 E-05$ & 6 \\
\hline \multicolumn{9}{|c|}{ Catenoidal panel } \\
\hline \multicolumn{9}{|c|}{$\eta=1:$ target frequency $f_{1 T}=590.557 \mathrm{~Hz}$} \\
\hline $\mathrm{MC}$ & 1.06122 & 0.90588 & 2.06831 & 2.50895 & 595.86858 & $3.9753 E-07$ & $8.9937 E-03$ & 55 \\
\hline GA & 0.08800 & -0.48390 & 13.11830 & 13.97850 & 590.77480 & $4.0135 E-07$ & $3.6832 E-04$ & 45 \\
\hline PSO & 0.09089 & -0.32061 & 18.33638 & 14.94431 & 590.11938 & $4.0129 E-07$ & $7.4152 E-04$ & 43 \\
\hline \multicolumn{9}{|c|}{ Elliptic paraboloid } \\
\hline \multicolumn{9}{|c|}{$\eta=0.5$ : target frequency $f_{1 T}=327.239 \mathrm{~Hz}$ and target displacement $w_{\max T}=u_{3 \max T}=1.9073 \cdot 10^{-6} \mathrm{~m}$} \\
\hline $\mathrm{MC}$ & 0.29917 & 0.02997 & 19.54311 & 2.65521 & 332.68799 & $1.8923 E-06$ & $1.2258 E-02$ & 43 \\
\hline GA & 0.08800 & -0.29910 & 3.01080 & 4.93650 & 327.62800 & $1.9075 E-06$ & $6.4729 E-04$ & 22 \\
\hline PSO & 0.25269 & -0.65120 & 13.49764 & 2.90612 & 327.39815 & $1.9065 E-06$ & $4.5339 E-04$ & 25 \\
\hline
\end{tabular}

TABLE 3: Perceptual relative errors in first frequtency $e_{f}=\left(\left(f_{1}-f_{1 T}\right) / f_{1 T}\right) \cdot 100$ and in maximum bending displacement $e_{w}=\left(\left(w_{\max }-\right.\right.$ $\left.\left.w_{\max T}\right) / w_{\max T}\right) \cdot 100$ with Monte Carlo, Particle Swarm Optimization, and Genetic Algorithm methods.

\begin{tabular}{lcccccccc}
\hline $\begin{array}{l}\text { Optimization } \\
\text { method }\end{array}$ & Square plate & Cylindrical panel & \multicolumn{2}{c}{ Conical shell } & Toroidal shell panel & \multicolumn{2}{c}{ Catenoidal panel } & \multicolumn{2}{c}{ Elliptic paraboloid } \\
& $e_{f}$ & $e_{w}$ & $e_{f}$ & $e_{w}$ & $e_{w}$ & $e_{f}$ & $e_{f}$ \\
\hline MC & -1.261 & -0.431 & 2.884 & -1.076 & 0.534 & 0.899 & 1.665 & -0.786 \\
GA & 0.024 & 0.188 & 0.070 & -0.029 & -0.053 & 0.037 & 0.119 & 0.010 \\
PSO & -0.016 & 0.059 & 0.118 & -0.046 & 0.008 & -0.074 & 0.049 & -0.042 \\
\hline
\end{tabular}

$L=3 \mathrm{~m}$, circumferential angle $\alpha_{2}=\vartheta \in\left[0,120^{\circ}\right]$, and a thickness $h=0.2 \mathrm{~m}$. It is subjected to a normal load $q_{n}^{+}=-10000 \mathrm{~Pa}$ at the top surface, and its boundary conditions are SFSF. On the contrary, the conical shell (Figure $4(\mathrm{c})$ ) has $R_{b}=1 \mathrm{~m}, L=3 \mathrm{~m}$, circumferential angle $\alpha_{2}=\vartheta \in\left[0,360^{\circ}\right], \alpha=30^{\circ}$, and a thickness $h=0.3 \mathrm{~m}$. Also the conical shell is subjected to a normal load $q_{n}^{+}=-10000 \mathrm{~Pa}$ at the top surface. The conical shell is clamped at the bottom and free at the top, so the boundary conditions are indicated by $\mathrm{CF}$. The cylindrical panel is a two-layered structure with $\mathrm{FGM}_{2\left(a^{(1)} / b^{(1)} / c^{(1)} / p^{(1)}\right)} / \mathrm{FGM}_{1\left(a^{(2)} / b^{(2)} / c^{(2)} / p^{(2)}\right)}$ lamination scheme, while the conical shell presents $\mathrm{FGM}_{1\left(a^{(1)} / b^{(1)} / c^{(1)} / p^{(1)}\right)} /$ 

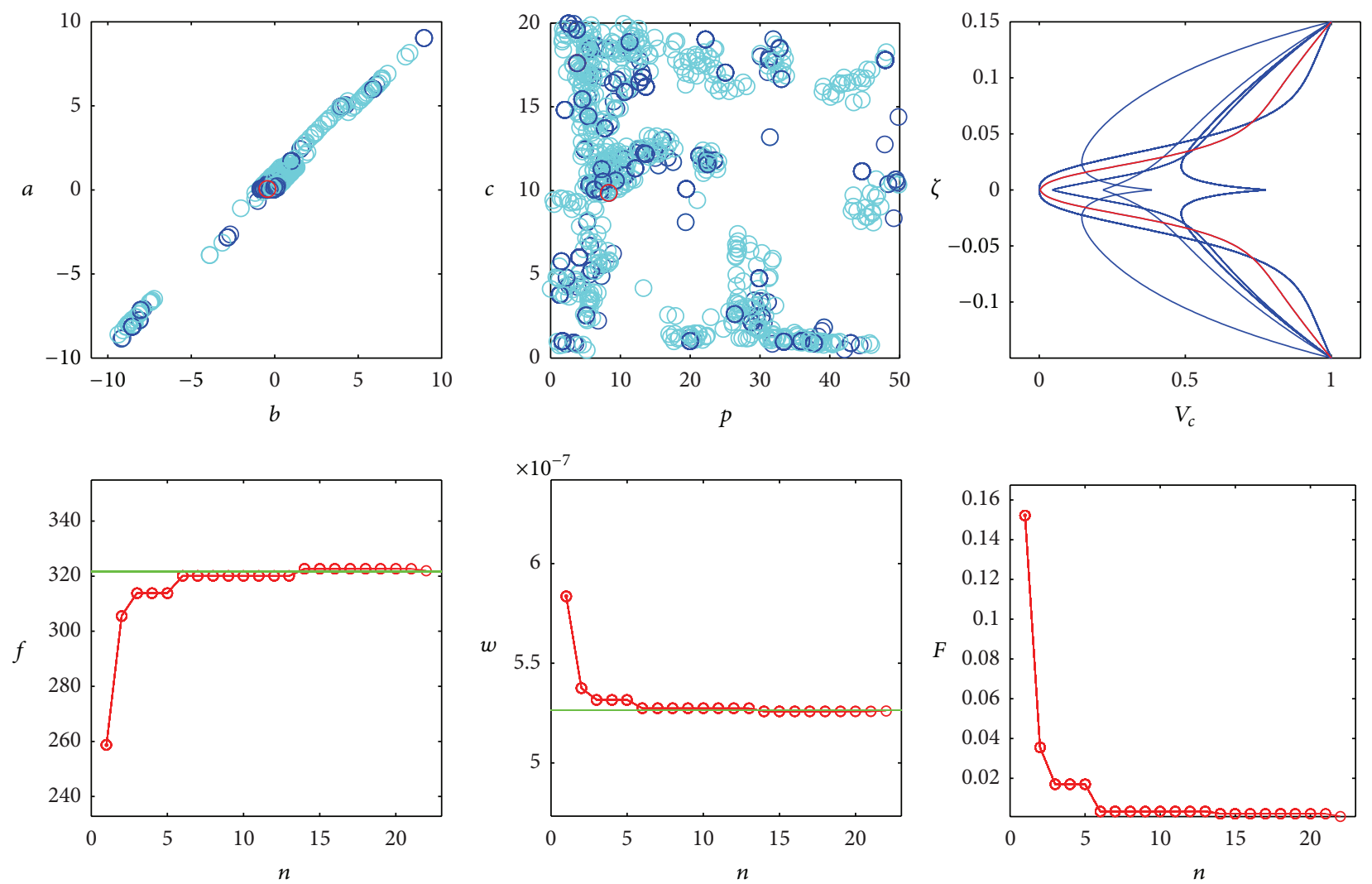

Figure 13: Conical shell: optimization by Particle Swarm Algorithm with $\eta=0.5$; final optimized frequency $f=322.049 \mathrm{~Hz}$ and final optimized displacement $w=5.2615 \cdot 10^{-7} \mathrm{~m}$.

$\mathrm{FGM}_{2\left(a^{(2)} / b^{(2)} / c^{(2)} / p^{(2)}\right)}$ lamination scheme. In these two cases the FGM four parameters of the two different power-law distributions are assumed to be equal to the two different laminae of the structure. Thus, only the four parameters $a=a^{(1)}=a^{(2)}, b=b^{(1)}=b^{(2)}, c=c^{(1)}=c^{(2)}$, and $p=p^{(1)}=p^{(2)}$ have to be determined by the optimization procedure.

As already reported in $[74,78]$, the position vector of a toroidal shell panel (Figure $4(\mathrm{~d})$ ) can be written as

$$
\begin{aligned}
\mathbf{r}\left(\alpha_{1}, \alpha_{2}\right)= & \left(R_{b}+R \sin \alpha_{1}\right) \cos \alpha_{2} \mathbf{e}_{1} \\
& -\left(R_{b}+R \sin \alpha_{1}\right) \sin \alpha_{2} \mathbf{e}_{2}+R \cos \alpha_{1} \mathbf{e}_{3}
\end{aligned}
$$

where $R_{b}$ is the shift of the circular meridian curve with respect to the axis of revolution $[23,43,44,47,68,69]$ and $R$ is the radius of the circular curve section of the toroidal shell panel. The toroidal structure is characterized by $R_{b}=9 \mathrm{~m}$, $R=3 \mathrm{~m}$, meridian angle $\alpha_{1}=\varphi \in\left[0,360^{\circ}\right]$, circumferential angle $\alpha_{2}=\vartheta \in\left[0,120^{\circ}\right]$, and a thickness $h=0.6 \mathrm{~m}$. Also the toroidal shell has CF boundary conditions, and the normal load $q_{n}^{+}=-10000 \mathrm{~Pa}$ is applied at its top surface. When a catenary curve is considered as a meridian curve of a revolution shell, the position vector of the catenoidal panel (Figure 4(e)) assumes the aspect

$$
\begin{aligned}
\mathbf{r}\left(\alpha_{1}, \alpha_{2}\right)= & \left(A \cosh \left(\operatorname{arcsinh}\left(\frac{1}{\tan \alpha_{1}}\right)\right)+R_{b}\right) \cos \alpha_{2} \mathbf{e}_{1} \\
& -\left(A \cosh \left(\operatorname{arcsinh}\left(\frac{1}{\tan \alpha_{1}}\right)\right)+R_{b}\right) \sin \alpha_{2} \mathbf{e}_{2} \\
& +A \operatorname{arcsinh}\left(\frac{1}{\tan \alpha_{1}}\right) \mathbf{e}_{3},
\end{aligned}
$$

where $A$ is the distance of the throat apex of the catenary curve. For further details about the geometry definition of the catenary curve, the reader might refer to [23, 43, 74, 78]. Regarding the catenoidal panel (Figure 4(e)) its geometrical properties are $A=2 \mathrm{~m}, B=2 \mathrm{~m}, D=1 \mathrm{~m}, R_{b}=0 \mathrm{~m}$, circumferential angle $\alpha_{2}=\vartheta \in\left[0,90^{\circ}\right]$, and a thickness $h=$ $0.5 \mathrm{~m}$. The catenoidal panel has CFCF boundary conditions, and it is subjected to a normal load $q_{n}^{+}=-10000 \mathrm{~Pa}$ at the top surface. The toroidal shell panel and catenary panel of revolution have the same three-layered lamination scheme $\mathrm{FGM}_{2\left(a^{(1)} / b^{(1)} / c^{(1)} / p^{(1)}\right)} / \mathrm{FGM}_{C} / \mathrm{FGM}_{1\left(a^{(3)} / b^{(3)} / c^{(3)} / p^{(3)}\right)}$. The lamination scheme presents the middle lamina of the structure made of ceramic isotropic material. Also, in these two cases the FGM parameters of the two different power-law distribution are assumed to be equal to the first and the third 

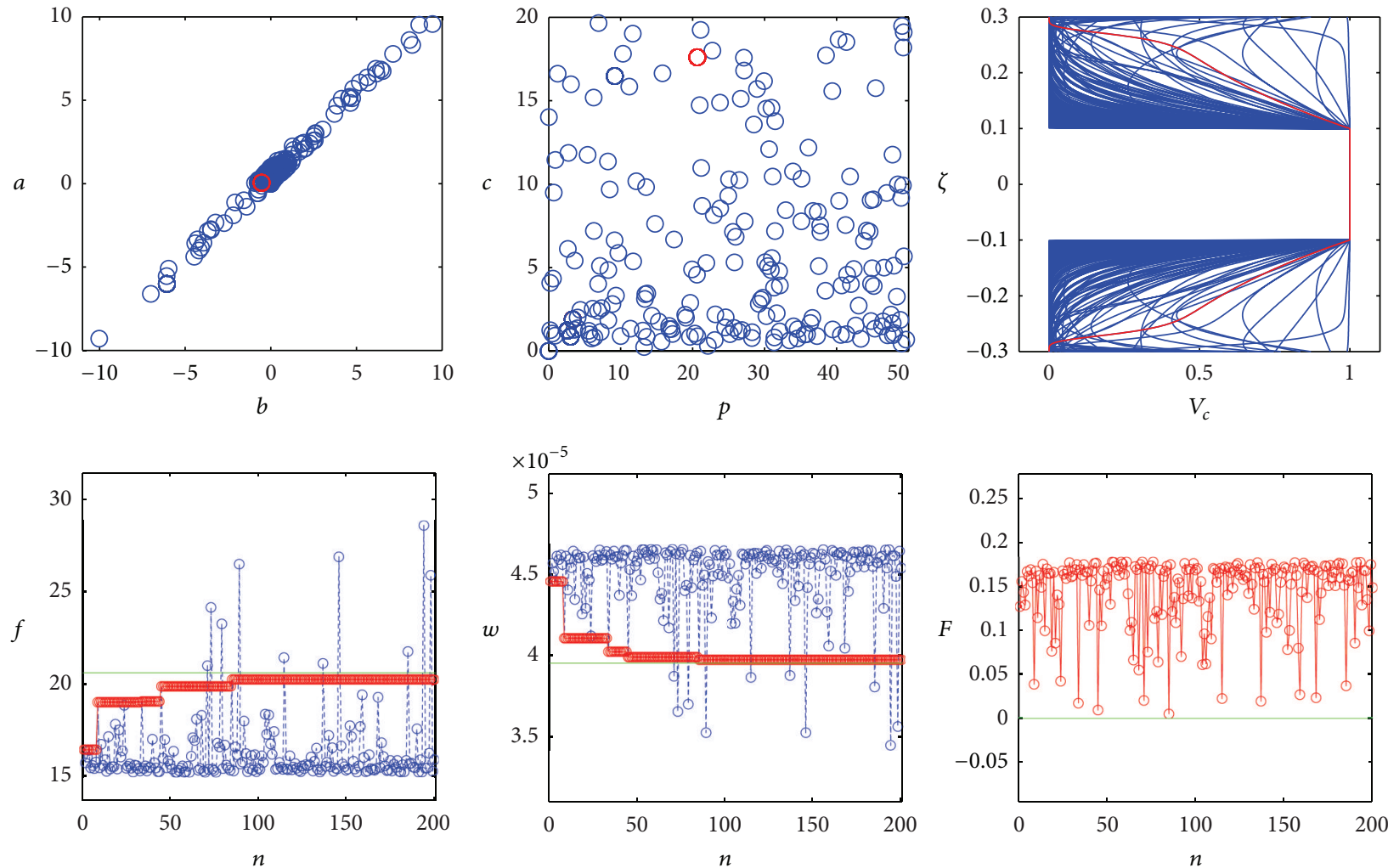

FIGURE 14: Toroidal shell panel: Monte Carlo optimization with $\eta=0$; final optimized displacement $w=3.9727 \cdot 10^{-5} \mathrm{~m}$.
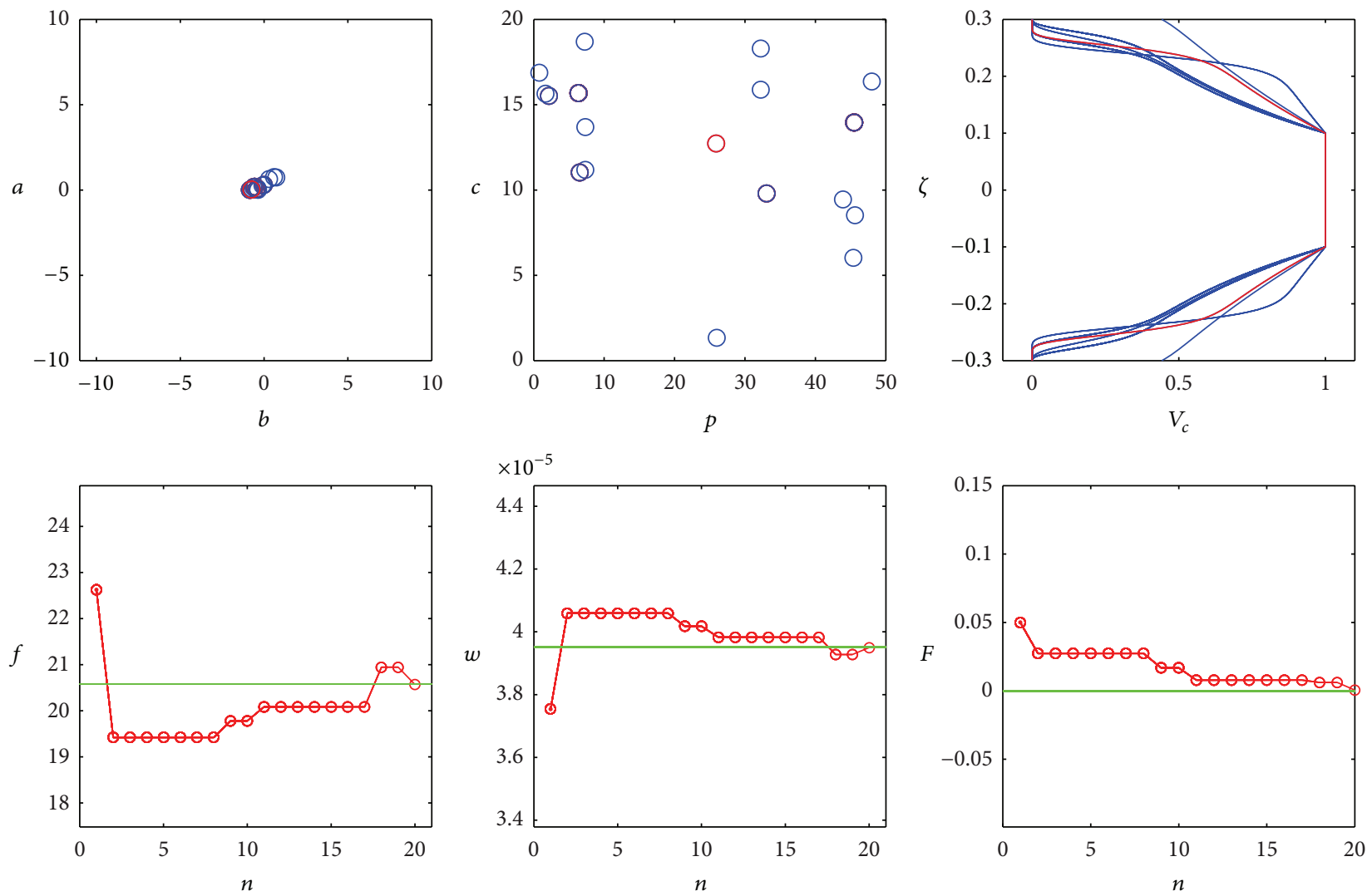

FIGURE 15: Toroidal shell panel: Genetic Algorithm optimization with $\eta=0$; final optimized displacement $w=3.9495 \cdot 10^{-5} \mathrm{~m}$. 

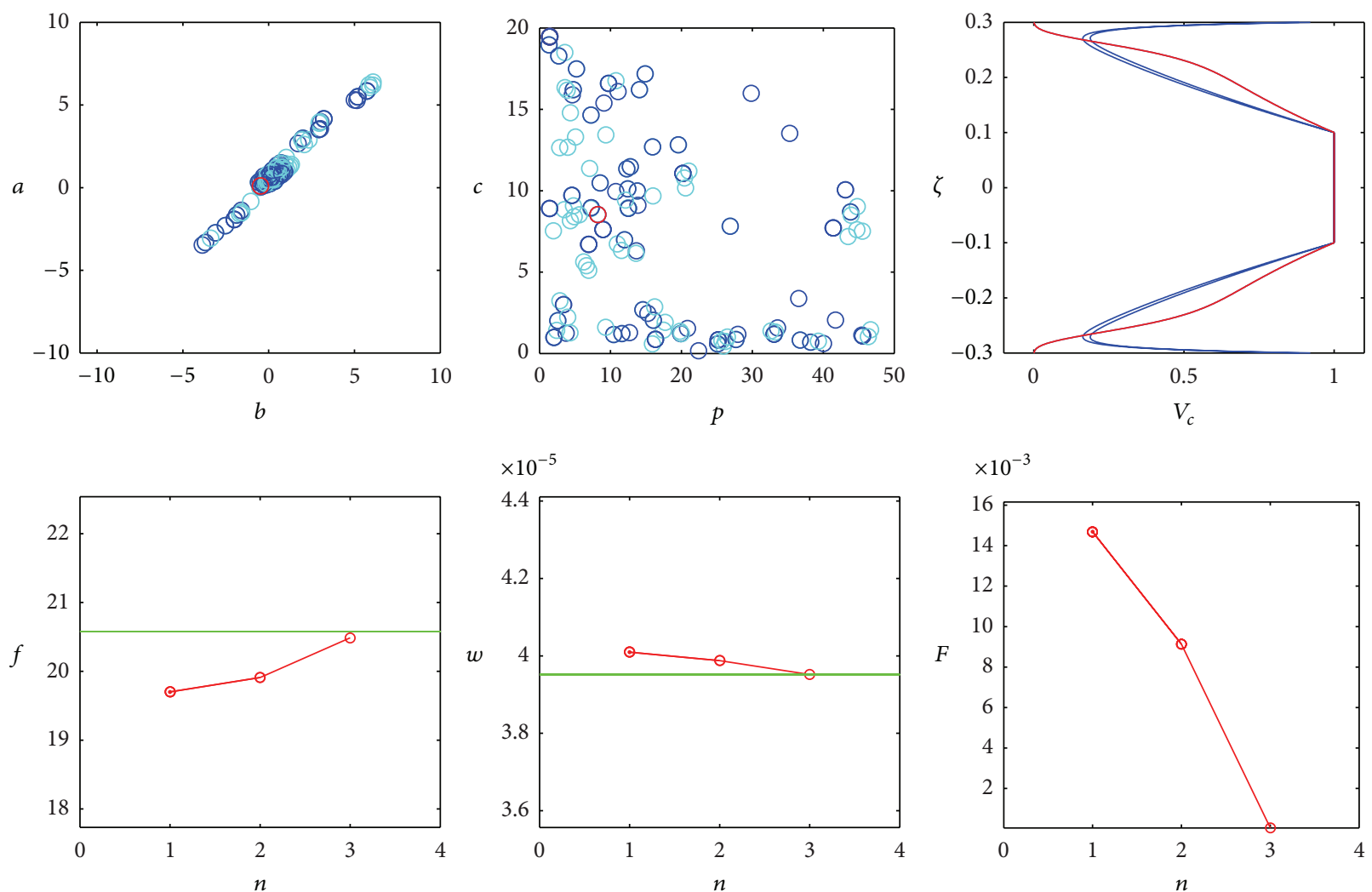

FIgURE 16: Toroidal shell panel: Particle Swarm Optimization with $\eta=0$; final optimized displacement $w=3.9519 \cdot 10^{-5} \mathrm{~m}$.
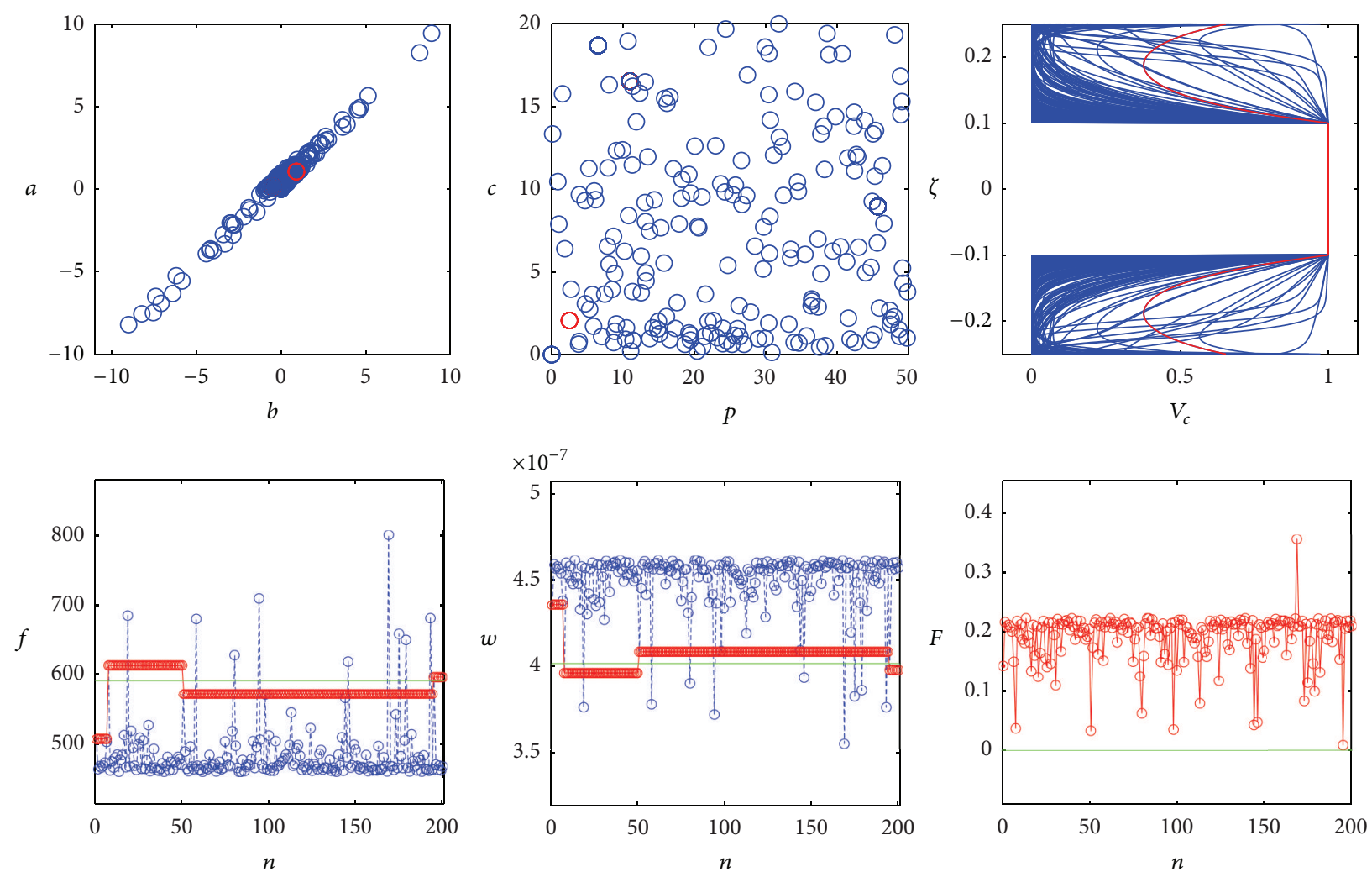

FIGURE 17: Catenoidal panel: Monte Carlo optimization with $\eta=1$; final optimized frequency $f=595.868 \mathrm{~Hz}$. 

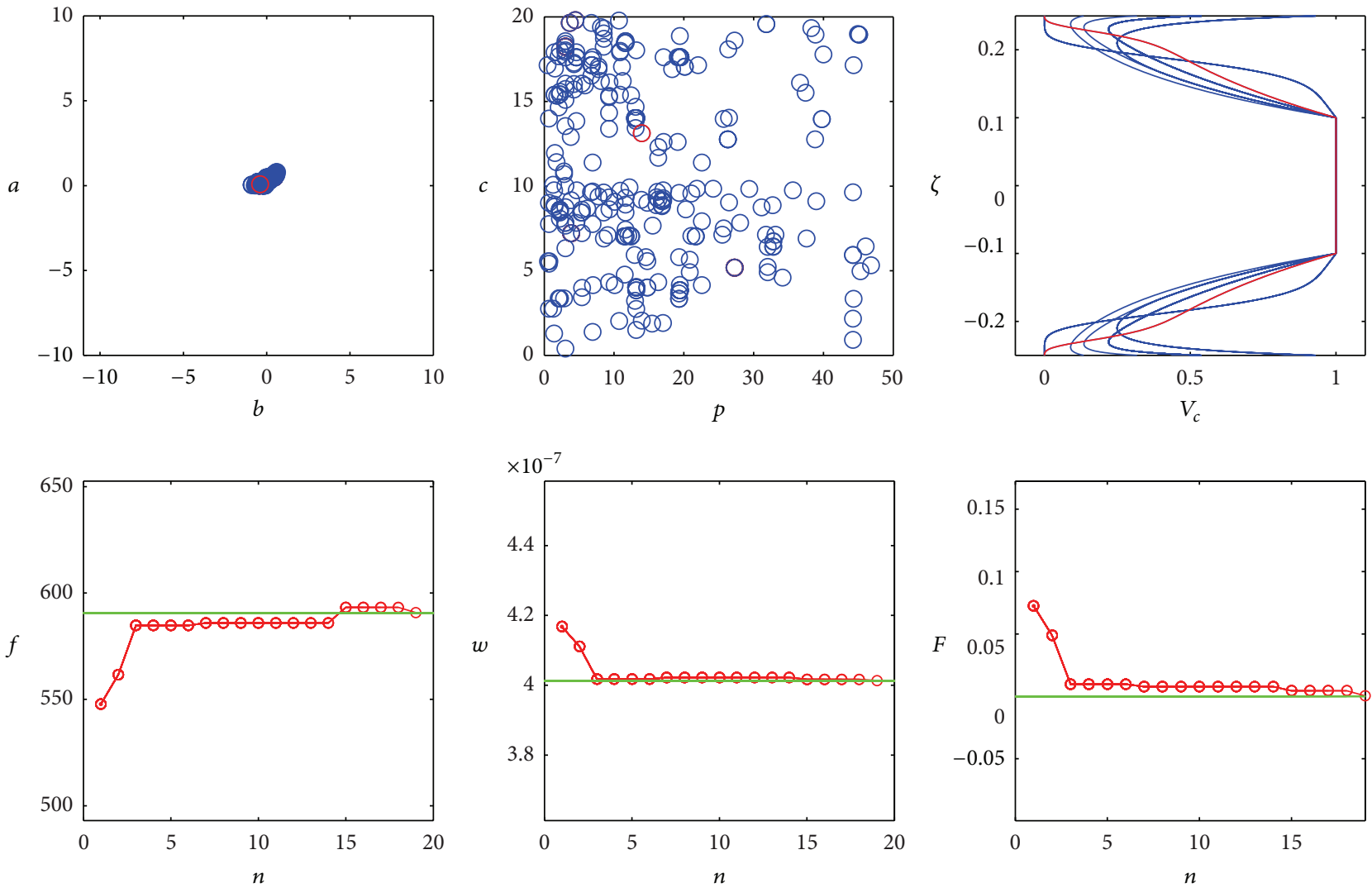

FIGURE 18: Catenoidal panel: Genetic Algorithm optimization with $\eta=1$; final optimized frequency $f=590.800 \mathrm{~Hz}$.

laminae of the structure. In this way, only the four parameters $a=a^{(1)}=a^{(3)}, b=b^{(1)}=b^{(3)}, c=c^{(1)}=c^{(3)}$, and $p=p^{(1)}=p^{(3)}$ have to be determined by the optimization procedure.

Finally, the position vector of the reference surface of the elliptic paraboloid is given in $[74,78]$ :

$$
\begin{aligned}
\mathbf{r}\left(\alpha_{1}, \alpha_{2}\right)= & \left(R_{0}^{\alpha_{1}}\left(\alpha_{1}\right)-x_{3}^{\alpha_{2}}\left(\alpha_{2}\right) \sin \alpha_{1}\right) \cos \alpha_{2} \mathbf{e}_{1} \\
& -R_{0}^{\alpha_{2}}\left(\alpha_{2}\right) \mathbf{e}_{2}+\left(x_{3}^{\alpha_{1}}\left(\alpha_{1}\right)+x_{3}^{\alpha_{2}}\left(\alpha_{2}\right) \cos \alpha_{1}\right) \mathbf{e}_{3} .
\end{aligned}
$$

If the generatrix parabola needs to be characterized (the other parabola can be defined analogously), it can be described by the following equation:

$$
\left(R_{0}^{\alpha_{1}}\right)^{2}-\kappa^{\alpha_{1}} x_{3}^{\alpha_{1}}=0,
$$

where $\kappa^{\alpha_{1}}=\left(A^{2}-D^{2}\right) / B$ is a characteristic parameter of the parabolic curve, $R_{0}^{\alpha_{1}}$ is the abscissa of a point of the parabola, and $x_{3}^{\alpha_{1}}$ is its ordinate in the generatrix plane of the parabolic curve. The abscissa $R_{0}^{\alpha_{1}}\left(\alpha_{1}\right)$ of the parabolic curve assumes the form

$$
R_{0}^{\alpha_{1}}\left(\alpha_{1}\right)=\frac{\kappa^{\alpha_{1}} \tan \alpha_{1}}{2} .
$$

The parameters describing the two parabolas of the elliptic paraboloid under consideration are $A=3 \mathrm{~m}, C=-3 \mathrm{~m}$, $D=0 \mathrm{~m}$, and $B=0.8 \mathrm{~m}$, and the thickness is $h=0.4 \mathrm{~m}$. The elliptic paraboloid is completely clamped at its four edges (CCCC), and it is subjected to a normal load $q_{n}^{+}=-10000 \mathrm{~Pa}$ at the top surface. The elliptic paraboloid is a single-layered structure with $\mathrm{FGM}_{2\left(a^{(1)} / b^{(1)} / c^{(1)} / p^{(1)}\right)}$ four-parameter powerlaw distribution. In this case, the four parameters $a=a^{(1)}, b=$ $b^{(1)}, c=c^{(1)}$, and $p=p^{(1)}$ have to be determined by the optimization procedure.

In Table 1 the first ten frequencies and maximum static deflections for the six structures of Figure 4 are presented. In particular, the two limit cases of functionally graded materials are considered. For the first case, named $\mathrm{FGM}_{C}$, the singlelayered structure is made of ceramic isotropic material, while for the second one, named $\mathrm{FGM}_{M}$, the single-layered structure is made of metal isotropic material. In the present work, it is assumed that the functionally graded material lamina is made of a mixture of ceramic and metal constituents: Silicon Nitride and Stainless Steel. Young's modulus, Poisson's ratio, and mass density for the Silicon Nitride are $E_{C}=322.27 \mathrm{GPa}$, $\nu_{\mathrm{C}}=0.24$, and $\rho_{\mathrm{C}}=2370 \mathrm{~kg} / \mathrm{m}^{3}$, and for the Stainless Steel are $E_{M}=207.78 \mathrm{GPa}, \nu_{M}=0.3177$, and $\rho_{\mathrm{M}}=8166 \mathrm{~kg} / \mathrm{m}^{3}$, respectively. 

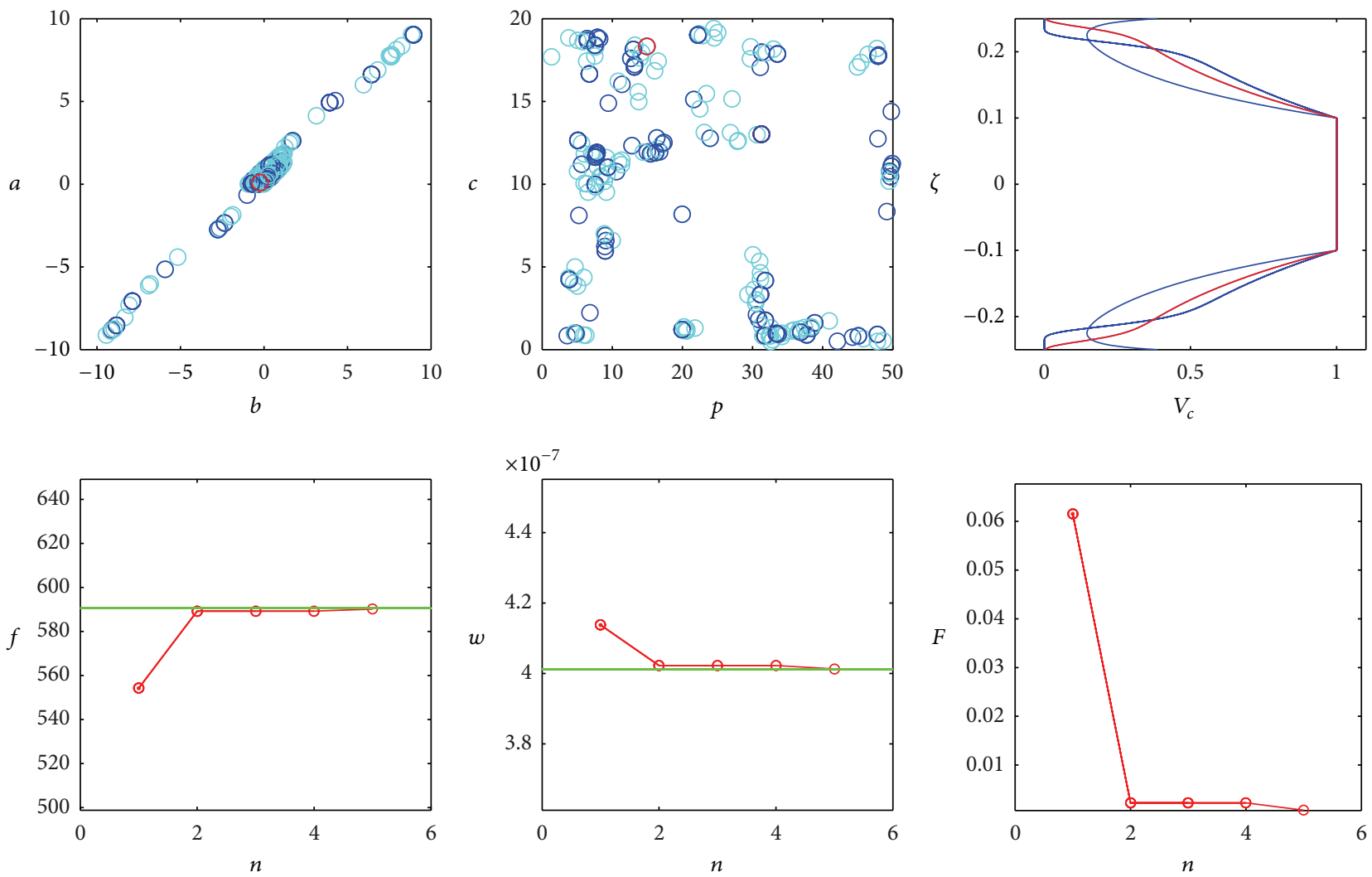

FIGURE 19: Catenoidal panel: Particle Swarm optimization with $\eta=1$; final optimized frequency $f=590.119 \mathrm{~Hz}$.

As described before, one of the goals of the present paper is to develop and test an operative methodology to support the design of FG materials. Several problems can be faced by the designer; in particular some of the most commonly occurred engineering needs are to avoid resonance frequency, to reduce or control the deformations, to increase the strength to weight ratio, to reduce stresses due to different dilatation properties of two materials, and so on. In this study, frequency and deformation and a combination of the two will be considered. The fitness function $F$ has been modelled to obtain a defined first resonance frequency target $\left(f_{1 T}\right)$ set by user, a maximum displacement target $\left(w_{\max T}=u_{3 \max T}\right)$ due to bending deformation, or to try to satisfy at the best both the requirements. In this latter case, the designer can decide to stress more the importance on one of the two requirements by using the coefficient $\eta$ which acts as a weight: in this way the fitness $F$ is still monodimensional but keeps into account two aspects, ranging $\eta$ from zero to one. According to the above considerations, the fitness expression $F$ can be so represented by the formula

$$
F=\eta \sqrt{\left(\frac{f_{1}-f_{1 T}}{f_{1 T}}\right)^{2}}+(1-\eta) \sqrt{\left(\frac{w_{\max }-w_{\max T}}{w_{\max T}}\right)^{2}} .
$$

It is worth to note that the minimization of the fitness $F$ is equivalent to obtain values of frequency $\left(f=f_{1}\right)$ and of displacement $\left(w=w_{\max }=u_{3 \max }\right)$ as close as possible to the desired ones $\left(f_{1 T}, w_{\max T}=u_{3 \max T}\right)$, and the ratio between the difference from the obtained value minus the target and the target value itself is used to make dimensionless frequencies and displacements. By this way, parameters with different magnitudes can be compared, allowing to equate items with unit of measurement defined by the experimenter depending on his practice. In the paper, this simple monoobjective fitness has been considered to privilege the simplicity and to suggest the use of this approach also in industrial applications, but more complex multiobjective fitness functions can be implemented depending on the specific case study and on the requirements and needs expressed by the designer.

Figures $5,6,7,8,9,10,11,12,13,14,15,16,17,18,19,20$, 21 , and 22 present the results of the optimization of the six structures described above. A mix of values of $\eta$ parameter has been chosen $(\eta=0,0.5,1)$, keeping it constant for each structure as to make possible the comparison of the optimization methods. Following formula (48), if $\eta=1$ only the frequency is optimized, while for $\eta=0$ only the displacement is considered; an intermediate value $\eta=0.5$ can be set to find the better compromise between the guess of both frequency and displacement. Each figure presents in the upper left corner the values of $a$ and $b$ parameter for each try; the best solution is represented by a red circle. In the upper centre there are the values of $c$ and $p$ parameters for each try with the best solution in red; in the upper left corner there is the material distribution in the thickness of the structure; an FGM made by one, two, or three layers 

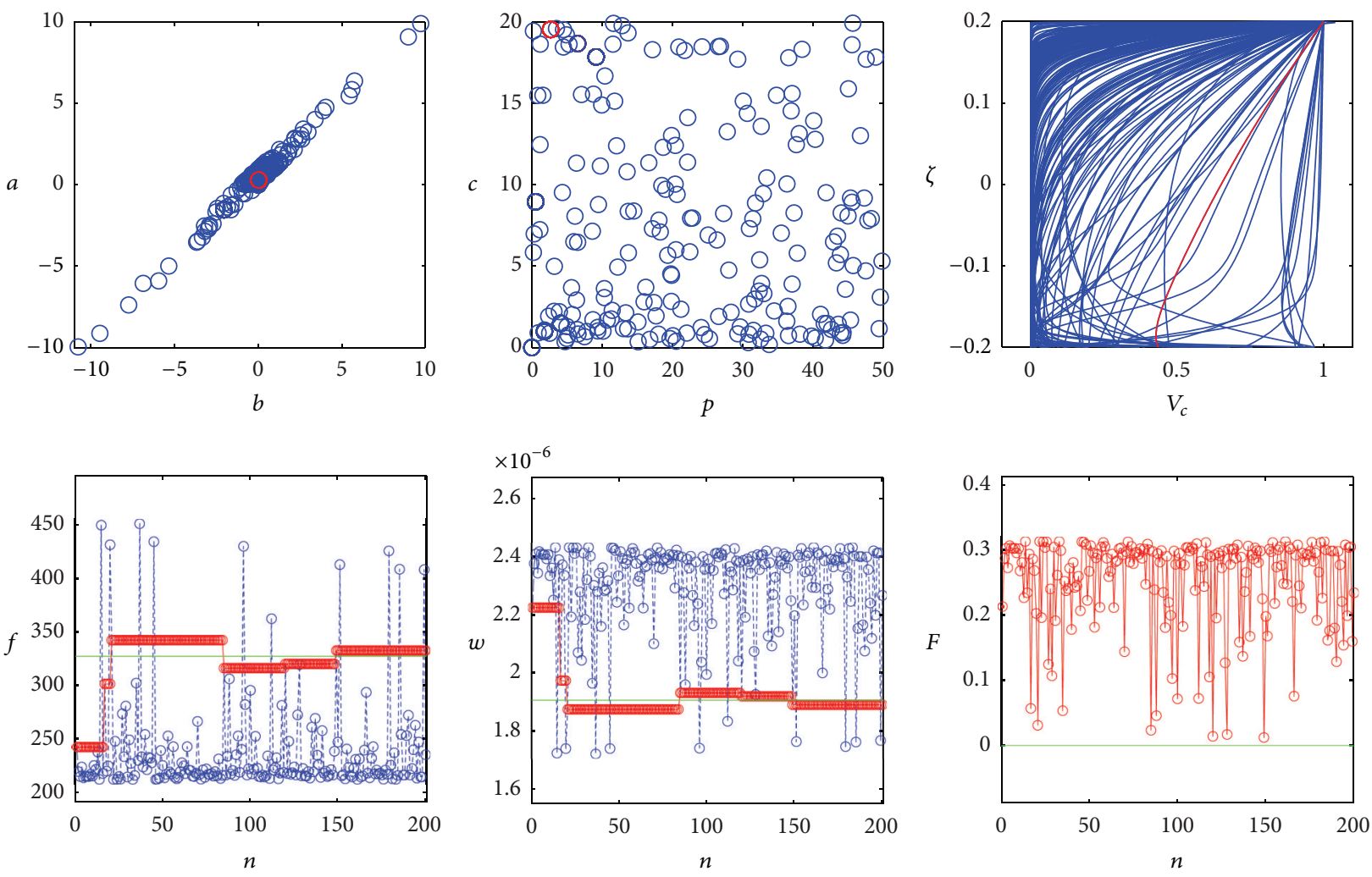

FIgURE 20: Elliptic paraboloid: Monte Carlo optimization with $\eta=0.5$; final optimized frequency $f=332.688 \mathrm{~Hz}$ and final optimized displacement $w=1.8923 \cdot 10^{-6} \mathrm{~m}$.

can be visualized, depending on the structure definition (e.g. rectangular plate presents only one layer; cylindrical panel presents two symmetric layers, while, for instance, the conical shell is made by a sandwich between an homogeneous material and two symmetric about the midplane layers of FG materials): the final optimized distribution is in red. The lower part of the figures from left to right presents the trend of frequency, displacement, and fitness in time. In case of Monte Carlo frequency and displacement of the single run are shown (with in blue the trend of the best solution found), while in case of Particle Swarm and Genetic Algorithms the frequency and the displacement referred to the best individual found at the moment are shown. A green line represents the target values for the frequency and the displacement, while in case of the fitness, the ideal value is obviously equal to zero.

Figures 5-7 present the optimization of a rectangular plate when the designer is interested in setting the first resonance frequency of the structure. The plate is made by only one FGM lamina, and a value of $\eta=1$ is set. Figures 810 depict the result of the optimization with MC, PSO, and GA of a cylindrical panel made by two symmetrical FGM distributions about the midplane; in this case $\eta=0$ is set since the need for a required maximum displacement is simulated. Figures 11-13 refer to a singly curved conical shell made by two FGM laminae, with a distribution symmetric about the midplane of the whole structure; in this case $\eta=0.5$ is set to consider an interest both in frequency and in maximum displacement. Figures 14-16 present a toroidal shell panel which is a doubly curved shell panel of revolution, made by three laminae: a homogeneous core and two external symmetrical FGM laminae as to simulate a typical sandwich structure. The interest is in maximum displacement, so that $\eta$ is set equal to zero. Figures 17-19 list the optimization trend of a catenoidal panel structure which can be defined as a doubly curved panel of revolution; the laminate is obtained by three layers also in this case, representing a typical sandwich structure; in this case the attention is focused on frequency optimization, so that $\eta=1$ is set. Finally, Figures $20-22$ present an elliptic paraboloid which is a completely doubly curved panel structure made by only one FGM lamina; considerations will be addressed both on frequency and displacement since $\eta=0.5$.

As to help the interpretation of the optimization process, the final results are collected in Table 2: the final value of the fitness provides an idea of the goodness of the results found. It is important to note that heuristic methods do not provide the best solution, but a suboptimal solution; in other words, sometimes, similar results of the fitness function can be found with several different set of parameters $a, b, c$, and $p$. The Genetic Algorithm has been run with a population of 30 individuals and the Particle Swarm with a number of particles of 30; these algorithms stop when a maximum number of runs are reached or no improvements are found in solution (or alternatively when the percentage error is less 

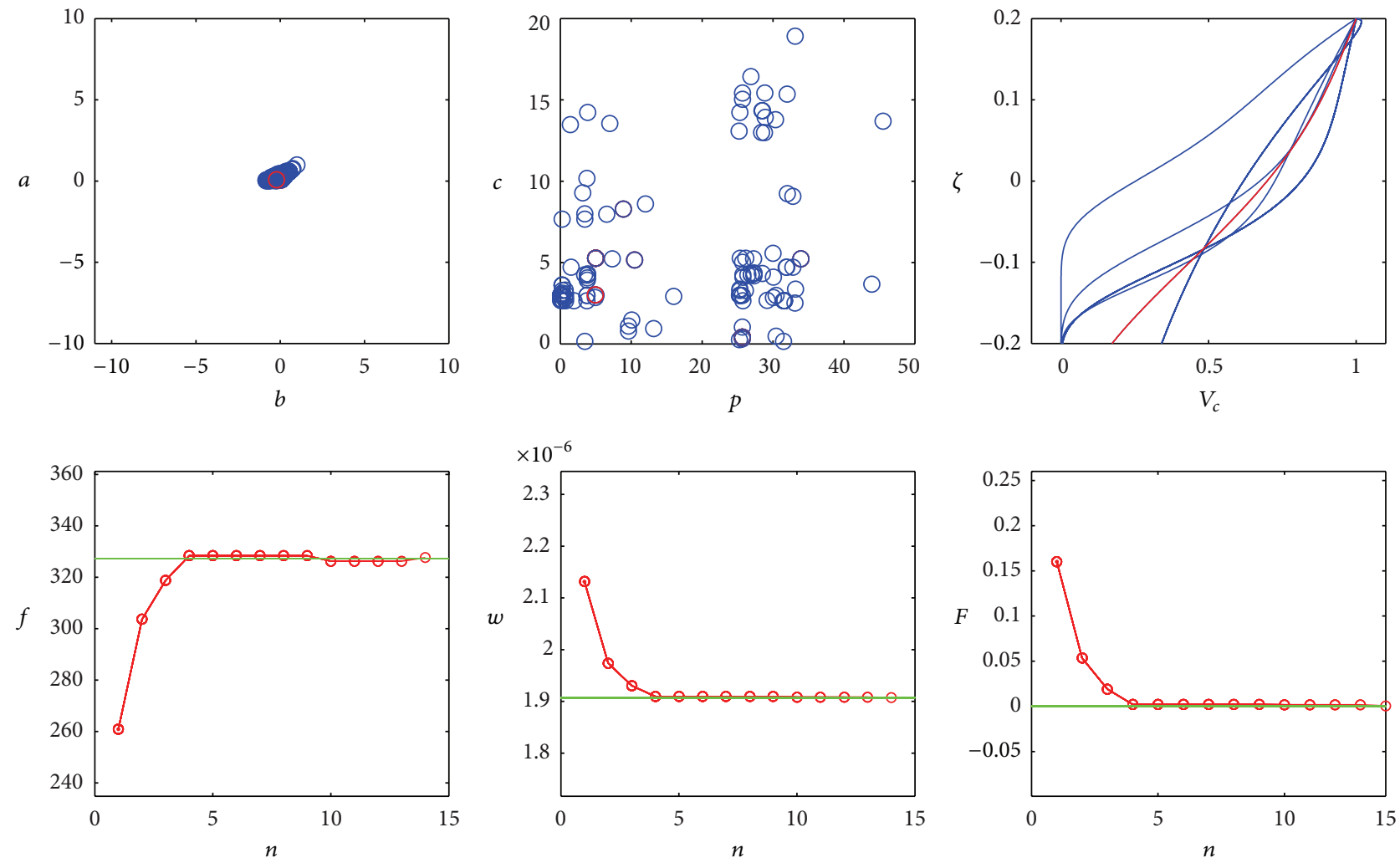

FIGURE 21: Elliptic paraboloid: Genetic Algorithm optimization with $\eta=0.5$; final optimized frequency $f=327.600 \mathrm{~Hz}$ and final optimized displacement $w=1.9075 \cdot 10^{-6} \mathrm{~m}$.

than the $0.5 \%)$. The Monte Carlo simulation has been run for 200 times for each optimization problem, in order to obtain a comparable number of runs with Genetic Algorithm and Particle Swarm Optimizers. A first comparison between methods can be performed based on Figure 23, in which there is a graph collecting final fitness versus computational time for each optimization performed. Values for Monte Carlo are in red triangles, Particle Swarm in blue squares, while Genetic Algorithm in red circles. As expected GA and PSO results are better than MC ones and provide data with good approximation in a relative short time, which is also compatible with the needs of conceptual design or Multidisciplinary Optimization. Simulations have been carried out on a Notebook equipped with an 8 GB RAM, a $2 \mathrm{GHz}$ core processor, and Windows 7 operating system. Table 3 lists the errors in frequency and in displacement (depending on the case study) for each simulation run; as can be seen by data provided, both GA and PSO present a similar efficiency. The two methods exploit different strategies to solve the optimization problem in this complex task: in this case, in fact, the domain is not continuous and some combinations of the four parameters $a, b, c$, and $p$ can lead to a distribution of metal and ceramics which is not consistent (percentage represented by a negative or complex or major than one number, which is physically unfeasible). As well described before in the paper, one of the challenges is that slight changes in one of the four parameters can lead to inconsistent solutions. When a particle of the PSO arrives to the border of the domain with a speed pointing to an inconsistent zone of the domain, it is needed to randomly redirect the particle towards an allowed zone, and this reduces the efficiency of the method. On the other hand, GA solves in a better way this problem since mutations and crossover help in exploring new zones of the domain in which the solution is feasible and the distribution percentage of the FGM distributions (ceramics and metal) is always a positive number between zero and one in all the thickness of the structure. Only a small part of the individuals of a generation should mutate because it slows down the convergence. After tests performed it seems that these two effects compensate and both PSO and GA present a very good behavior in optimization. The comparison between random optimization (MC) with heuristic methods (GA, PSO) shows a different relative efficiency of $\mathrm{MC}$ when applied to cases in which only frequency or displacement is studied or when both are considered. In the first case the difference in precision obtained by MC with respect to GA and PSO is quite small, while this difference increases dramatically when both frequency and displacement are considered: it can be inferred that when the fitness function deals with only one parameter also the MC alone can be used, while in case of fitness involving more parameters an heuristic or semi heuristic method provides better results. 

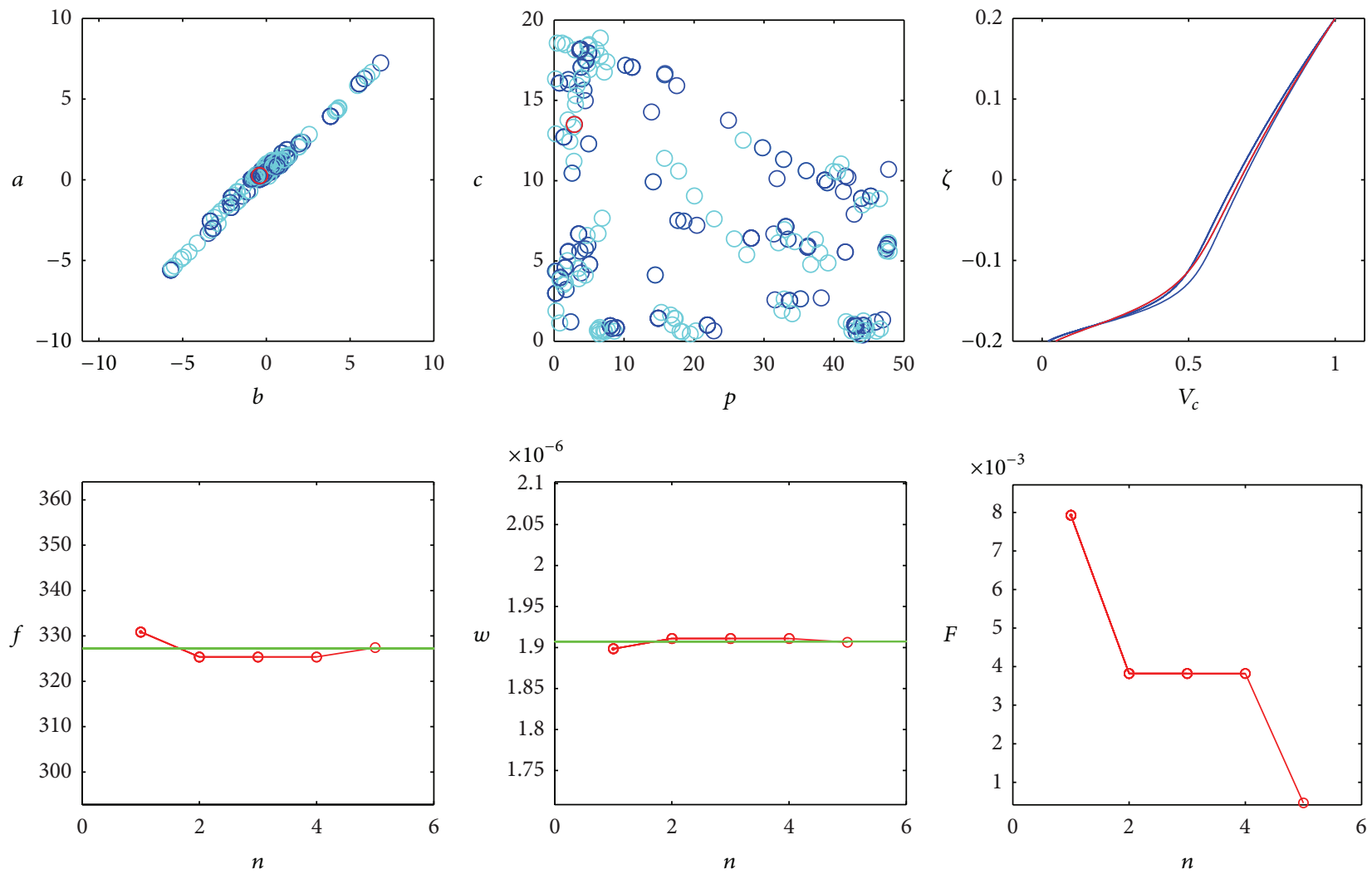

FIGURE 22: Elliptic paraboloid: Particle Swarm Optimization with $\eta=0.5$; final optimized frequency $f=327.398 \mathrm{~Hz}$ and final optimized displacement $w=1.9065 \cdot 10^{-6} \mathrm{~m}$.

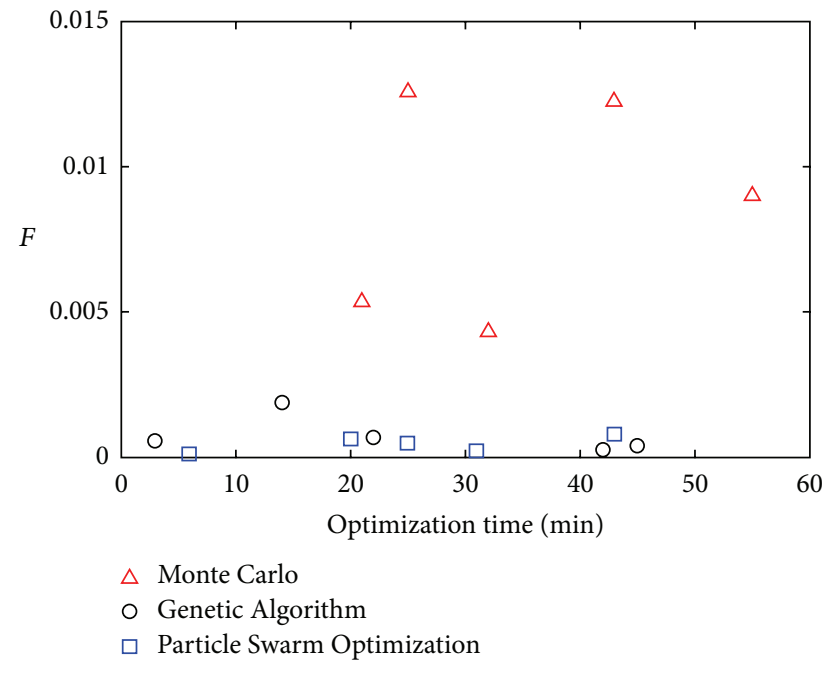

FIGURE 23: Computational optimization time [in minutes] versus fitness for MC (triangle), GA (circle), and PSO (square).

\section{Conclusion Remarks and Summary}

Functionally graded material applications are increasing due to their potentialities in problems related to aggressive environments, thermal properties, and special structural needs.
Theoretical frameworks have been developed to solve the static and dynamic analysis of this type of structures. In the present paper, the Generalized Differential Quadrature method has been presented as a mean to investigate the static and dynamic analysis of functionally graded and laminated composite doubly curved shells and panels. The adopted shell theory is the First-order Shear Deformation Theory. In particular, the Toorani-Lakis theory has been used as a starting point to obtain the governing equations for shells. The 2D equilibrium equations have been discretized with the GDQ method through standard linear algebraic and eigenvalue problems. Thanks to the mathematical approach, the analysis of an FGM shell or panel can be provided. The material distribution can be expressed by a four-parameter powerlaw. However, the main drawback of this approach from a designer perspective is that it is impossible to analytically relate the set of the four parameters to static or dynamic performances of the structures. An approach based on the application of optimization algorithms to the problem has been carried out to evaluate its suitability to the topic. Due to the domain of the four parameters which includes zones in which no solutions can be found, the optimization algorithms have been modified and tested. Genetic Algorithm, Particle Swarm Optimization, and Monte Carlo algorithms have been selected for tests and applied to six different geometrical structures optimizing the first frequency, maximum bending displacement, or a mix of the two. Results obtained show 
the convergence of the optimization for GA and PSO, which provides better results than MC for similar computational effort. In particular, GA and PSO are more precise when both frequency and displacement are considered at the same time. The herein developed methodology can dramatically help the designer in the definition of the mix ceramics/metal which can present the requested properties.

\section{Acknowledgments}

This research was supported by the Italian Ministry Education, Universities and Research (MIUR).

\section{References}

[1] S. Timoshenko and S. Woinowsky-Krieger, Theory of Plates and Shells, McGraw-Hill, New York, NY, USA, 1959.

[2] W. Flügge, Stresses in Shells, Springer, Berlin, Germany, 1960.

[3] A. L. Gol'denveizer, Theory of Elastic Thin Shells, Pergamon Press, Oxford, UK, 1961.

[4] V. V. Novozhilov, Thin Shell Theory, P. Noordhoff, Groningen, The Netherlands, 1964.

[5] V. Z. Vlasov, "General Theory of Shells and Its Application in Engineering, NASA-TT-F-99," 1964.

[6] S. A. Ambartusumyan, "Theory of Anisotropic Shells, NASATT-F-118," 1964.

[7] H. Kraus, Thin Elastic Shells, John Wiley \& Sons, New York, NY, USA, 1967.

[8] A. W. Leissa, "Vibration of Plates, NASA-SP-160," 1969.

[9] A. W. Leissa, "Vibration of Shells, NASA-SP-288," 1973.

[10] S. Markuš, The Mechanics of Vibrations of Cylindrical Shells, Elsevier, New York, NY, USA, 1988.

[11] E. Ventsel and T. Krauthammer, Thin Plates and Shells, Marcel Dekker, New York, NY, USA, 2001.

[12] W. Soedel, Vibrations of Shells and Plates, Marcel Dekker, New York, NY, USA, 2004.

[13] E. Reissner, "The effect of transverse shear deformation on the bending of elastic plates," Journal of Applied Mechanics, vol. 12, pp. 66-77, 1945.

[14] R. D. Mindlin, "Influence of rotatory inertia and shear on flexural vibration of isotropic, elastic plates," Journal of Applied Mechanics, vol. 18, pp. 31-38, 1951.

[15] P. L. Gould, Finite Element Analysis of Shells of Revolution, Pitman Publishing, London, UK, 1984.

[16] P. L. Gould, Analysis of Plates and Shells, Prentice-Hall, New York, NY, USA, 1999.

[17] A. W. Leissa and J.-D. Chang, "Elastic deformation of thick, laminated composite shells," Composite Structures, vol. 35, no. 2, pp. 153-170, 1996.

[18] M. S. Qatu, "Accurate equations for laminated composite deep thick shells," International Journal of Solids and Structures, vol. 36, no. 19, pp. 2917-2941, 1999.

[19] M. S. Qatu, Vibration of Laminated Shells and Plates, Elsevier, New York, NY, USA, 2004.

[20] M. H. Toorani and A. A. Lakis, "General equations of anisotropic plates and shells including transverse shear deformations, rotary inertia and initial curvature effects," Journal of Sound and Vibration, vol. 237, no. 4, pp. 561-615, 2000.
[21] M. H. Toorani and A. A. Lakis, "Free vibrations of non-uniform composite cylindrical shells," Nuclear Engineering and Design, vol. 236, no. 17, pp. 1748-1758, 2006.

[22] J. N. Reddy, Mechanics of Laminated Composites Plates and Shells, CRC Press, New York, NY, USA, 2003.

[23] F. Tornabene, Meccanica delle Strutture a Guscio in Materiale Composito. Il Metodo Generalizzato di Quadratura Differenziale, Esculapio, Bologna, Italy, 2012.

[24] E. Carrera, S. Brischetto, and P. Nali, Plates and Shells for Smart Structures: Classical and Advanced Theories for Modeling and Analysis, John Wiley \& Sons, New York, NY, USA, 2011.

[25] E. Carrera, "Historical review of Zig-Zag theories for multilayered plates and shells," Applied Mechanics Reviews, vol. 56, no. 3, pp. 287-308, 2003.

[26] C. Shu, Differential Quadrature and Its Application in Engineering, Springer, Berlin, Germany, 2000.

[27] C. W. Bert and M. Malik, "Differential quadrature method in computational mechanics: a review," Applied Mechanics Reviews, vol. 49, no. 1, pp. 1-27, 1996.

[28] K. M. Liew, J. B. Han, and Z. M. Xiao, "Differential quadrature method for thick symmetric cross-ply laminates with first-order shear flexibility," International Journal of Solids and Structures, vol. 33, no. 18, pp. 2647-2658, 1996.

[29] C. Shu and H. Du, "Free vibration analysis of laminated composite cylindrical shells by DQM," Composites B, vol. 28, no. 3, pp. 267-273, 1997.

[30] K. M. Liew and T. M. Teo, "Modeling via differential quadrature method: three-dimensional solutions for rectangular plates," Computer Methods in Applied Mechanics and Engineering, vol. 159, no. 3-4, pp. 369-381, 1998.

[31] F.-L. Liu and K. M. Liew, "Differential quadrature element method: a new approach for free vibration analysis of polar Mindlin plates having discontinuities," Computer Methods in Applied Mechanics and Engineering, vol. 179, no. 3-4, pp. 407423, 1999.

[32] S. Moradi and F. Taheri, "Delamination buckling analysis of general laminated composite beams by differential quadrature method," Composites B, vol. 30, no. 5, pp. 503-511, 1999.

[33] T. Y. Ng, L. Hua, K. Y. Lam, and C. T. Loy, "Parametric instability of conical shells by the generalized differential quadrature method," International Journal for Numerical Methods in Engineering, vol. 44, no. 6, pp. 819-837, 1999.

[34] T. C. Fung, "Solving initial value problems by differential quadrature method-part 1: first-order equations," International Journal for Numerical Methods in Engineering, vol. 50, no. 6, pp. 1411-1427, 2001.

[35] T. C. Fung, "Solving initial value problems by differential quadrature method. II. Second- and higher-order equations," International Journal for Numerical Methods in Engineering, vol. 50, no. 6, pp. 1429-1454, 2001.

[36] K. M. Liew, T. Y. Ng, and J. Z. Zhang, "Differential quadraturelayerwise modeling technique for three-dimensional analysis of cross-ply laminated plates of various edge-supports," Computer Methods in Applied Mechanics and Engineering, vol. 191, no. 35, pp. 3811-3832, 2002.

[37] T. Y. Wu, Y. Y. Wang, and G. R. Liu, "Free vibration analysis of circular plates using generalized differential quadrature rule," Computer Methods in Applied Mechanics and Engineering, vol. 191, no. 46, pp. 5365-5380, 2002.

[38] G. Karami and P. Malekzadeh, "Static and stability analyses of arbitrary straight-sided quadrilateral thin plates by DQM," 
International Journal of Solids and Structures, vol. 39, no. 19, pp. 4927-4947, 2002.

[39] J. Yang and H.-S. Shen, "Nonlinear bending analysis of shear deformable functionally graded plates subjected to thermomechanical loads under various boundary conditions," Composites B, vol. 34, no. 2, pp. 103-115, 2003.

[40] K. M. Liew, J. Z. Zhang, C. Li, and S. A. Meguid, "Threedimensional analysis of the coupled thermo-piezoelectromechanical behaviour of multilayered plates using the differential quadrature technique," International Journal of Solids and Structures, vol. 42, no. 14, pp. 4239-4257, 2005.

[41] E. Viola and F. Tornabene, "Vibration analysis of damaged circular arches with varying cross-section," SID Structural Integrity and Durability, vol. 1, no. 2, pp. 155-169, 2005.

[42] E. Viola and F. Tornabene, "Vibration analysis of conical shell structures using GDQ method," Far East Journal of Applied Mathematics, vol. 25, pp. 23-39, 2006.

[43] F. Tornabene, Modellazione e soluzione di strutture a Guscio in materiale anisotropo [Ph.D. thesis], University of BolognaDISTART Department, Bologna, Italy, 2007.

[44] F. Tornabene and E. Viola, "Vibration analysis of spherical structural elements using the GDQ method," Computers and Mathematics with Applications, vol. 53, no. 10, pp. 1538-1560, 2007.

[45] E. Viola, M. Dilena, and F. Tornabene, "Analytical and numerical results for vibration analysis of multi-stepped and multidamaged circular arches," Journal of Sound and Vibration, vol. 299, no. 1-2, pp. 143-163, 2007.

[46] A. Marzani, F. Tornabene, and E. Viola, "Nonconservative stability problems via generalized differential quadrature method," Journal of Sound and Vibration, vol. 315, no. 1-2, pp. 176-196, 2008.

[47] F. Tornabene and E. Viola, "2-D solution for free vibrations of parabolic shells using generalized differential quadrature method," European Journal of Mechanics, A/Solids, vol. 27, no. 6, pp. 1001-1025, 2008.

[48] A. Alibeigloo and R. Madoliat, "Static analysis of cross-ply laminated plates with integrated surface piezoelectric layers using differential quadrature," Composite Structures, vol. 88, no. 3, pp. 342-353, 2009.

[49] F. Tornabene, "Free vibration analysis of functionally graded conical, cylindrical shell and annular plate structures with a four-parameter power-law distribution," Computer Methods in Applied Mechanics and Engineering, vol. 198, no. 37-40, pp. 29112935, 2009.

[50] F. Tornabene and E. Viola, "Free vibrations of four-parameter functionally graded parabolic panels and shells of revolution," European Journal of Mechanics, vol. 28, no. 5, pp. 991-1013, 2009.

[51] F. Tornabene and E. Viola, "Free vibration analysis of functionally graded panels and shells of revolution," Meccanica, vol. 44, no. 3, pp. 255-281, 2009.

[52] F. Tornabene, E. Viola, and D. J. Inman, "2-D differential quadrature solution for vibration analysis of functionally graded conical, cylindrical and annular shell structures," Journal of Sound and Vibration, vol. 328, no. 3, pp. 259-290, 2009.

[53] E. Viola and F. Tornabene, "Free vibrations of three parameter functionally graded parabolic panels of revolution," Mechanics Research Communications, vol. 36, no. 5, pp. 587-594, 2009.

[54] Y. Li and Z. Shi, "Free vibration of a functionally graded piezoelectric beam via state-space based differential quadrature," Composite Structures, vol. 87, no. 3, pp. 257-264, 2009.
[55] A. Alibeigloo and V. Nouri, "Static analysis of functionally graded cylindrical shell with piezoelectric layers using differential quadrature method," Composite Structures, vol. 92, no. 8, pp. 1775-1785, 2010.

[56] A. Andakhshideh, S. Maleki, and M. M. Aghdam, "Non-linear bending analysis of laminated sector plates using Generalized Differential Quadrature," Composite Structures, vol. 92, no. 9, pp. 2258-2264, 2010.

[57] M. Farid, P. Zahedinejad, and P. Malekzadeh, "Threedimensional temperature dependent free vibration analysis of functionally graded material curved panels resting on two-parameter elastic foundation using a hybrid semi-analytic, differential quadrature method," Materials and Design, vol. 31, no. 1, pp. 2-13, 2010.

[58] Sh. Hosseini-Hashemi, H. Akhavan, H. R. D. Taher, N. Daemi, and A. Alibeigloo, "Differential quadrature analysis of functionally graded circular and annular sector plates on elastic foundation," Materials and Design, vol. 31, no. 4, pp. 1871-1880, 2010.

[59] Sh. Hosseini-Hashemi, M. Fadaee, and M. Es'Haghi, "A novel approach for in-plane/out-of-plane frequency analysis of functionally graded circular/annular plates," International Journal of Mechanical Sciences, vol. 52, no. 8, pp. 1025-1035, 2010.

[60] P. Malekzadeh and A. Alibeygi Beni, "Free vibration of functionally graded arbitrary straight-sided quadrilateral plates in thermal environment," Composite Structures, vol. 92, no. 11, pp. 2758-2767, 2010.

[61] O. Sepahi, M. R. Forouzan, and P. Malekzadeh, "Large deflection analysis of thermo-mechanical loaded annular FGM plates on nonlinear elastic foundation via DQM," Composite Structures, vol. 92, no. 10, pp. 2369-2378, 2010.

[62] B. Sobhani Aragh and M. H. Yas, "Three-dimensional free vibration of functionally graded fiber orientation and volume fraction cylindrical panels," Materials and Design, vol. 31, no. 9, pp. 4543-4552, 2010.

[63] F. Tornabene, A. Marzani, E. Viola, and I. Elishakoff, "Critical flow speeds of pipes conveying fluid by the generalized differential quadrature method," Advances in Theoretical and Applied Mechanics, vol. 3, no. 3, pp. 121-138, 2010.

[64] M. H. Yas and B. Sobhani Aragh, “Three-dimensional analysis for thermoelastic response of functionally graded fiber reinforced cylindrical panel," Composite Structures, vol. 92, no. 10, pp. 2391-2399, 2010.

[65] O. Sepahi, M. R. Forouzan, and P. Malekzadeh, "Thermal buckling and postbuckling analysis of functionally graded annular plates with temperature-dependent material properties," Materials and Design, vol. 32, no. 7, pp. 4030-4041, 2011.

[66] A. H. Sofiyev and N. Kuruoglu, "Natural frequency of laminated orthotropic shells with different boundary conditions and resting on the Pasternak type elastic foundation," Composites $B$, vol. 42, no. 6, pp. 1562-1570, 2011.

[67] F. Tornabene, "Free vibrations of laminated composite doublycurved shells and panels of revolution via the GDQ method," Computer Methods in Applied Mechanics and Engineering, vol. 200, no. 9-12, pp. 931-952, 2011.

[68] F. Tornabene, "2-D GDQ solution for free vibrations of anisotropic doubly-curved shells and panels of revolution," Composite Structures, vol. 93, no. 7, pp. 1854-1876, 2011.

[69] F. Tornabene, "Free vibrations of anisotropic doubly-curved shells and panels of revolution with a free-form meridian resting on Winkler-Pasternak elastic foundations," Composite Structures, vol. 94, no. 1, pp. 186-206, 2011. 
[70] F. Tornabene, A. Liverani, and G. Caligiana, "FGM and laminated doubly curved shells and panels of revolution with a free-form meridian: a 2-D GDQ solution for free vibrations," International Journal of Mechanical Sciences, vol. 53, no. 6, pp. 446-470, 2011.

[71] F. Tornabene, A. Liverani, and G. Caligiana, "Laminated composite rectangular and annular plates: a GDQ solution for static analysis with a posteriori shear and normal stress recovery," Composites B, vol. 43, no. 4, pp. 1847-1872, 2012.

[72] F. Tornabene, A. Liverani, and G. Caligiana, "Static analysis of laminated composite curved shells and panels of revolution with a posteriori shear and normal stress recovery using generalized differentialquadrature method," International Journal of Mechanical Sciences, vol. 61, pp. 71-87, 2012.

[73] F. Tornabene, A. Liverani, and G. Caligiana, "FGM and laminated doubly curved shells and panels of revolution with a free-form meridian: a 2-D GDQ solution for free vibrations," International Journal of Mechanical Sciences, vol. 53, no. 6, pp. 446-470, 2011.

[74] E. Viola, F. Tornabene, and N. Fantuzzi, "General higherorder shear deformation theories for the free vibration analysis of completely doubly-curved laminated shells and panels," Composite Structures, vol. 95, pp. 639-666, 2013.

[75] F. Tornabene and E. Viola, "Static analysis of functionally graded doubly-curved shells and panels of revolution," Meccanica, vol. 48, no. 4, pp. 901-930, 2013.

[76] F. Tornabene and A. Ceruti, "Free-form laminated doublycurved shells and panels of revolution resting on WinklerPasternak elastic foundations: a 2-D GDQ solution for static and free vibration analysis," World Journal of Mechanics, vol. 3, pp. 1-25, 2013.

[77] E. Viola, F. Tornabene, and N. Fantuzzi, "Static analysis of completely doubly-curved laminated shells and panels using general higher-order shear deformation theories," Composite Structures, vol. 101, pp. 59-93, 2013.

[78] B. Chen and L. Tong, "Sensitivity analysis of heat conduction for functionally graded materials," Materials and Design, vol. 25, no. 8, pp. 663-672, 2004.

[79] H.-S. Shen, Functionally Graded Materials: Nonlinear Analysis of Plates and Shells, CRC Press, New York, NY, USA, 2009.

[80] P. Malekzadeh, S. A. Shahpari, and H. R. Ziaee, "Threedimensional free vibration of thick functionally graded annular plates in thermal environment," Journal of Sound and Vibration, vol. 329, no. 4, pp. 425-442, 2010.

[81] X. Zhao and K. M. Liew, "Free vibration analysis of functionally graded conical shell panels by a meshless method," Composite Structures, vol. 93, no. 2, pp. 649-664, 2011.

[82] P. Malekzadeh, M. R. G. Haghighi, and M. M. Atashi, "Free vibration analysis of elastically supported functionally raded annular plates subjected to thermal environment," Meccanica, vol. 46, no. 5, pp. 893-913, 2011.

[83] A. H. Akbarzadeh, M. Abbasi, S. K. Hosseini zad, and M. R. Eslami, "Dynamic analysis of functionally graded plates using the hybrid Fourier-Laplace transform under thermomechanical loading," Meccanica, vol. 46, no. 6, pp. 1373-1392, 2011.

[84] S. K. Jalali, M. H. Naei, and A. Poorsolhjouy, "Thermal stability analysis of circular functionally graded sandwich plates of variable thickness using pseudo-spectral method," Materials and Design, vol. 31, no. 10, pp. 4755-4763, 2010.

[85] E. Jomehzadeh, A. R. Saidi, and S. R. Atashipour, "An analytical approach for stress analysis of functionally graded annular sector plates," Materials and Design, vol. 30, no. 9, pp. 36793685, 2009.

[86] Y. Fu, P. Zhang, and F. Yang, "Interlaminar stress distribution of composite laminated plates with functionally graded fiber volume fraction," Materials and Design, vol. 31, no. 6, pp. 29042915, 2010.

[87] N. Wattanasakulpong, B. G. Prusty, D. W. Kelly, and M. Hoffman, "Free vibration analysis of layered functionally graded beams with experimental validation," Materials \& Design, vol. 36, pp. 182-190, 2012.

[88] W. Montealegre Rubio, G. H. Paulino, and E. C. Nelli Silva, "Analysis, manufacture and characterization of $\mathrm{Ni} / \mathrm{Cu}$ functionally graded structures," Materials \& Design, vol. 41, pp. 255-265, 2012.

[89] A. Shaghaghi Moghaddam, M. Alfano, and R. Ghajar, "Determining the mixed mode stress intensity factors of surface cracks in functionally graded hollow cylinders," Materials \& Design, vol. 43, pp. 475-484, 2013.

[90] R. Moradi-Dastjerdi, M. Foroutan, and A. Pourasghar, "Dynamic analysis of functionally graded material cylinders under an impact load by a mesh-free method," Acta Mechanica, vol. 219, no. 3-4, pp. 281-290, 2011.

[91] M. Heshmati and M. H. Yas, "Vibrations of non-uniform functionally graded MWCNTs-polystyrene nanocomposite beams under action of moving load," Materials \& Design, vol. 46, pp. 206-218, 2013.

[92] M. Yas, S. Kamarian, J. E. Jam, and A. Pourasghar, "Weight minimization of functionally graded structures using ICA and ANN," International Journal of Advanced Scientific and Technical Research, vol. 2, pp. 679-696, 2012.

[93] J. E. Jam, S. Kamarian, and A. Pourasghar, "Application of ICA and ANN for optimization of functionally graded conical shells," International Journal of Engineering Trends in Engineering and Development, vol. 2, pp. 171-189, 2012.

[94] F. Glover and M. Laguna, Tabu Search, Kluwer Academic, Boston, Mass, USA, 1997.

[95] S. Kirkpatrick, C. D. Gelatt Jr., and M. P. Vecchi, "Optimization by simulated annealing," Science, vol. 220, no. 4598, pp. 671-680, 1983.

[96] H. H. Hoos and T. Stutzle, Stochastic Local Search: Foundations and Applications, Elsevier, Amsterdam, The Netherlands, 2005.

[97] A. Colorni, M. Dorigo, and V. Manniezzo, "An investigation of some properties of an ant algorithm," in Parallel Problem Solving from Nature 2, R. Manner and B. Manderick, Eds., pp. 509-520, North-Holland, Amsterdam, The Netherlands, 1992.

[98] M. Dorigo and G. Di Caro, "Ant colony optimization: a new meta-heuristic," in Proceedings of the 1999 Congress on Evolutionary Computation (CEC'99), vol. 2, pp. 1477-1484, 1999.

[99] D. E. Goldberg, Genetic Algorithms in Search, Optimization and Machine Learning, Addison-Wesley, Boston, Mass, USA, 1989.

[100] J. Koza, Genetic Programming: on the Programming of Computers by Means of Natural Selection, MIT Press, Cambridge, UK, 1992.

[101] R. Beasly, D. Bull, and R. Martin, "An overview on genetic algorithms: part I, fundamentals," University Computing, vol. 15, pp. 58-69, 1993.

[102] A. Ceruti, G. Caligiana, and F. Persiani, "Comparative evaluation of different optimization methodologies for the design of UAVs having shape obtained by hot wire cutting techniques," International Journal on Interactive Design and Manufacturing, 2012. 
[103] H. V. Hultmann Ayala and L. Dos Santos Coelho, "Tuning of PID controller based on a multi-objective genetic algorithm applied to a robotic manipulator," Expert Systems With Applications, vol. 39, no. 10, pp. 8968-8974, 2012.

[104] K. S. Tang, K. F. Man, S. Kwong, and Q. He, "Genetic algorithms and their applications," IEEE Signal Processing Magazine, vol. 13, no. 6, pp. 22-37, 1996.

[105] M. M. Karim, K. Suzuki, and H. Kai, "Optimal design of hydrofoil and marine propeller using micro-genetic algorithm," Journal of Naval Architecture and Marine Engineering, vol. 1, pp. 47-61, 2004.

[106] A. Konak, D. W. Coit, and A. E. Smith, "Multi-objective optimization using genetic algorithms: a tutorial," Reliability Engineering and System Safety, vol. 91, no. 9, pp. 992-1007, 2006.

[107] J. H. Holland, Adaptation in Natural and Artificial Systems, University of Michigan Press, Ann Arbor, Mich, USA, 1975.

[108] D. Kaur and M. M. Murugappan, "Performance enhancement in solving traveling salesman problem using hybrid genetic algorithm," in Proceedings of the Annual Conference of the North American Fuzzy Information Processing Society (NAFIPS '08), May 2008.

[109] E. Verdú, M. J. Verdú, L. M. Regueras, J. P. De Castro, and R. García, "A genetic fuzzy expert system for automatic question classification in a competitive learning environment," Expert Systems With Applications, vol. 39, no. 8, pp. 7471-7478, 2012.

[110] R. Storn and K. Price, "Differential evolution-a simple and efficient heuristic for global optimization over continuous spaces," Journal of Global Optimization, vol. 11, no. 4, pp. 341359, 1997.

[111] J. Kennedy and R. Eberhart, "Particle swarm optimization," in Proceedings of the 1995 IEEE International Conference on Neural Networks. Part 1 (of 6), pp. 1942-1948, December 1995.

[112] R. Poli, "An analysis of publications on particle swarm optimization applications," Technical Report CSM-469, Department of Computer Science, University of Essex, Essex, UK, 2007.

[113] Y. Shi and R. C. Eberhart, "Parameter selection in particle swarm optimization," in Proceedings of the 7th International Conference on Evolutionary Programming VII (EP '98), pp. 591600, 1998.

[114] L. Zhang, E. Dong, and Y. Xing, "Steering trapezoid mechanism design based on Monte Carlo method," in Proceedings of the International Conference on Electronic \& Mechanical Engineering and Information Technology, 2011.

[115] B. Birge, "PSOt: a particle swarm optimization toolbox for use with MATLAB," in Proceedings of the IEEE Swarm Intelligence Symposium (SIS '03), pp. 182-186, Indianapolis, Ind, USA, 2003.

[116] X. Hu, L. Wang, and Y. Zhong, "An improved particle swarm optimization algorithm for site index curve model," in Proceedings 2011 International Conference on Business Management and Electronic Information (BMEI '11), vol. 3, pp. 838-842, 2011.

[117] J. Yoo and P. Hajela, "Immune network simulations in multicriterion design," Structural and Multidisciplinary Optimization, vol. 18, no. 2-3, pp. 85-94, 1999.

[118] C. A. Coello Coello and N. C. Cortés, "Use of emulations of the immune system to handle constraints in evolutionary algorithms," in Proceedings of the Intelligent Engineering Systems Through Artificial Neural Networks (ANNIE '01), C. H. Dagli, A. L. Buczak, J. Ghosh et al., Eds., vol. 11, pp. 141-146, ASME Press, 2001.

[119] Y. T. Hsiao, C. L. Chuang, J. A. Jiang, and C. C. Chien, "A novel optimization algorithm: space gravitational optimization," in
Proceedings of the International Conference on Systems, Man and Cybernetics, pp. 2323-2328, Waikoloa, Hawaii, USA, October 2005.

[120] B. Abdi, H. Mozafari, A. Ayob, and R. Kohandel, "Imperialist competitive algorithm and its application in optimization of laminated composite structures," European Journal of Scientific Research, vol. 55, no. 2, pp. 174-187, 2011.

[121] H. Shah-Hosseini, "The intelligent water drops algorithm: a nature-inspired swarm-based optimization algorithm," International Journal of Bio-Inspired Computation, vol. 1, pp. 71-79, 2008.

[122] H. Duan, S. Liu, and X. Lei, "Air robot path planning based on intelligent water drops optimization," in Proceedings of the 2008 International Joint Conference on Neural Networks (IJCNN '08), pp. 1397-1401, June 2008.

[123] K. Mosegaard and M. Sambridge, "Monte Carlo analysis of inverse problems," Inverse Problems, vol. 18, no. 3, pp. R29-R54, 2002.

[124] G. S. Fishman, Monte Carlo: Concepts, Algorithms, and Applications, Springer, New York, NY, USA, 1995.

[125] S. Ghahramani, Fundamentals of Probability Theory, Prentice Hall, Upper Saddle River, NJ, USA, 2000.

[126] E. M. T. Hendrix and N. J. Olieman, "The smoothed Monte Carlo method in robustness optimization," Optimization Methods and Software, vol. 23, no. 5, pp. 717-729, 2008.

[127] K. Madani and J. R. Lund, "A Monte-Carlo game theoretic approach for Multi-Criteria decision making under uncertainty," Advances in Water Resources, vol. 34, no. 5, pp. 607-616, 2011. 


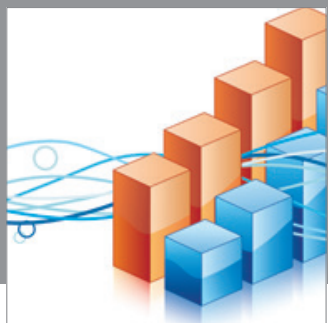

Advances in

Operations Research

mansans

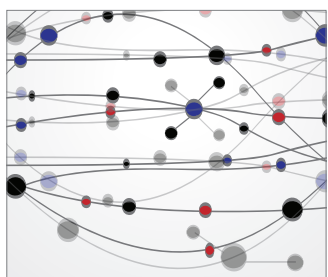

The Scientific World Journal
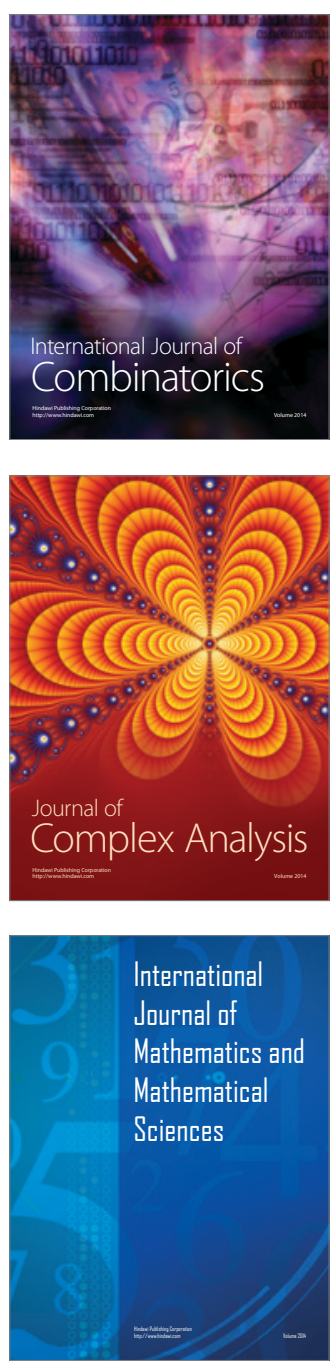
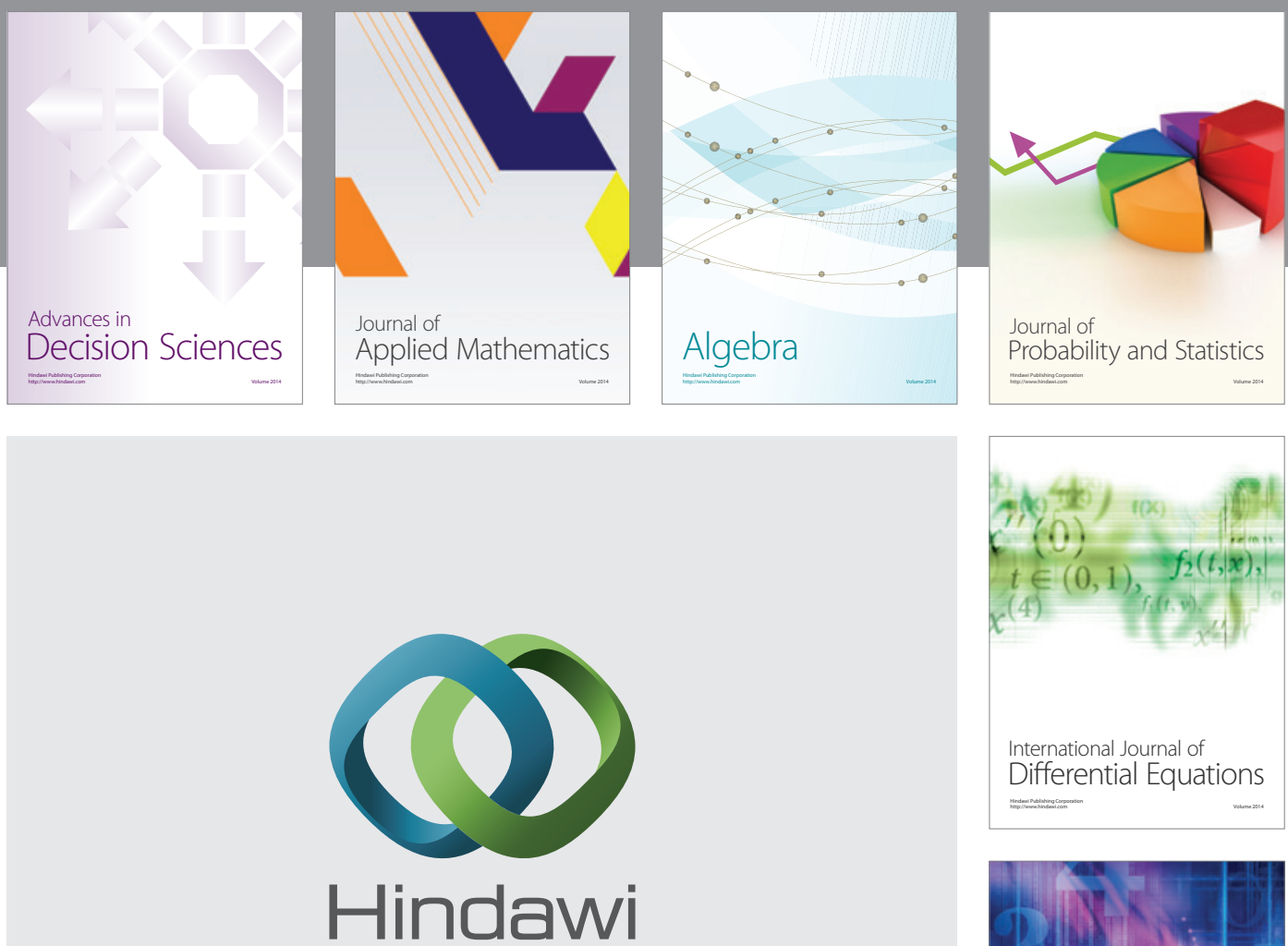

Submit your manuscripts at http://www.hindawi.com
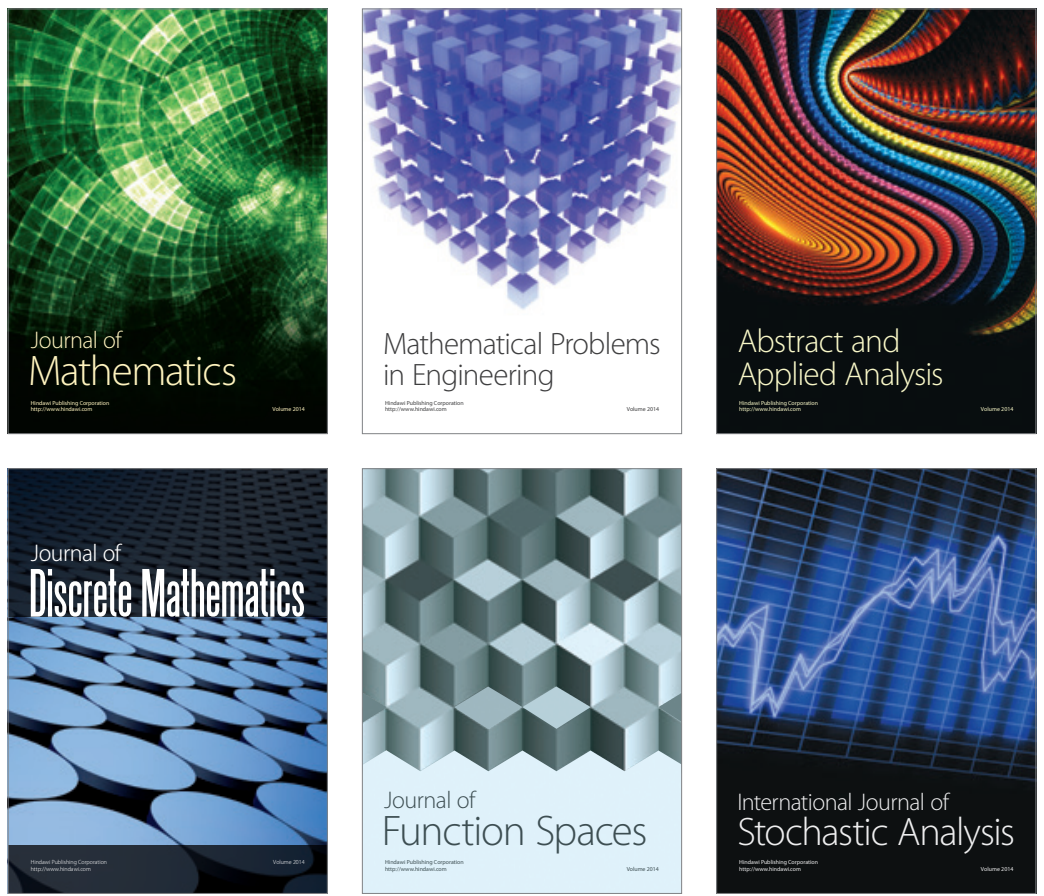

Journal of

Function Spaces

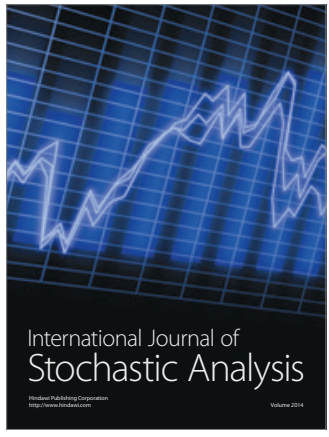

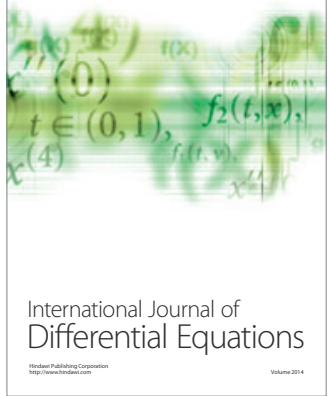
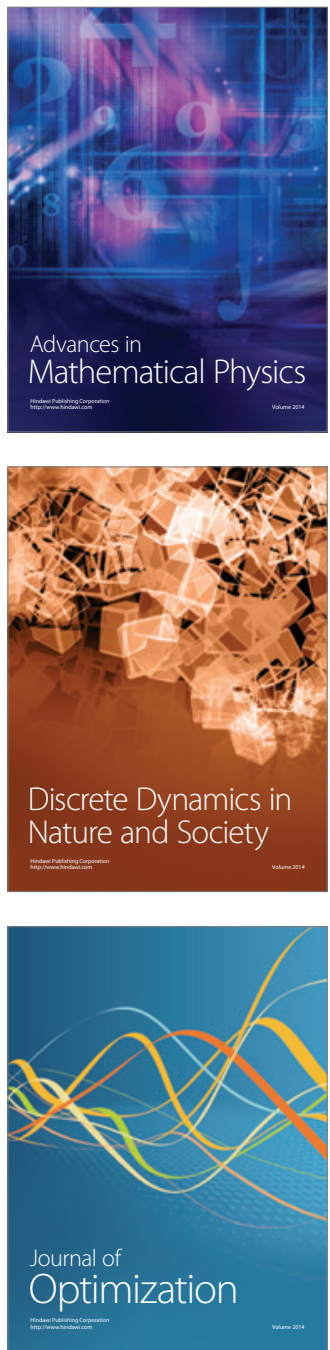